\title{
Atmospheric Transport Modeling and Input Data for Phase I of the Hanford Environmental Dose Reconstruction Project
}

J. V. Ramsdell

K. W. Burk

July 1991
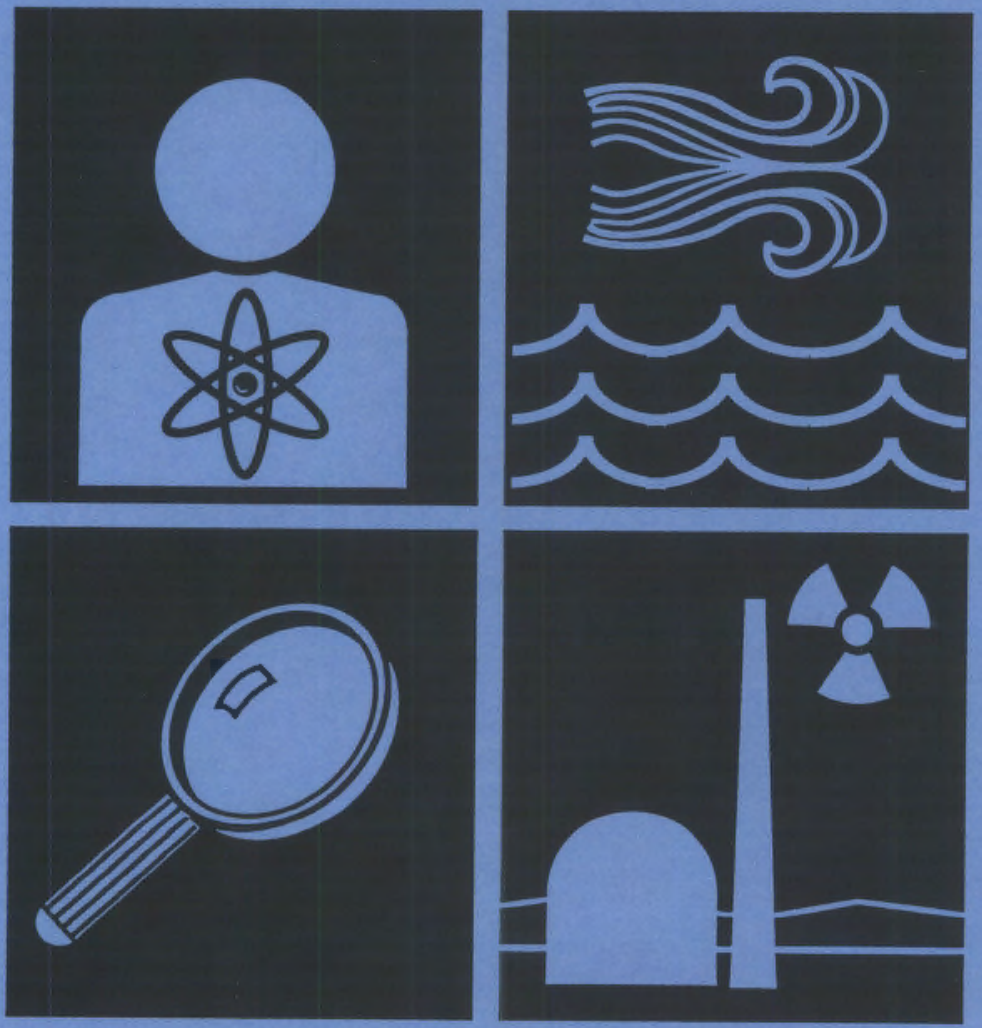

Prepared for the Technical Steering Panel 


\section{DISCLAIMER}

This report was prepared under the direction of the HANFORD ENVIRONMENTAL DOSE RECONSTRUCTION PROJECT Technical Steering Panel by Battelle Memorial Institute's Pacific Northwest Laboratories operating the Pacific Northwest Laboratory for the U.S. Department of Energy (DOE). While funding for the work was provided by DOE, the work is not under DOE direction or control. The views and opinions of the authors expressed in this document do not necessarily reflect those of the United States Covernment or any agency thereof. Reference herein to any specific commercial product, process or service by trade name, trademark, manufacturer or otherwise does not necessarily constitute or imply its endorsement, recommendation or favoring by the U.S. Government or any agency thereof, nor by Battelle Memorial Institute.

Printed in the United States of America

Available to DOE and DOE contractors from the

Office of Scientific and Technical Information, P.O. Box 62, Oak Ridge, TN 37031; prices available from (615) 576-8401. FTS 626-8401.

Available to the public from the National Technical Information Service, U.S. Department of Commerce, 5285 Port Royal Rd., Springfield, VA 22161. 
PNL - 7199 HEDR

UC -707

ATMOSPHERIC TRANSPORT MODELING AND INPUT DATA FOR PHASE I OF THE HANFORD ENVIRONMENTAL DOSE RECONSTRUCTION PROJECT

J. V. Ramsdell

K. W. Burk

July 1991

Prepared for the

Technical Steering Panel

Pacific Northwest Laboratory

Richland, washington 99352 


\section{ATMOSPHERIC TRANSPORT MODELING AND INPUT DATA FOR PHASE I OF THE HANFORD ENVIRONMENTAL DOSE RECONSTRUCTION PROJECT}

July 1991

This document has been reviewed and approved by the Technical Steering Panel.
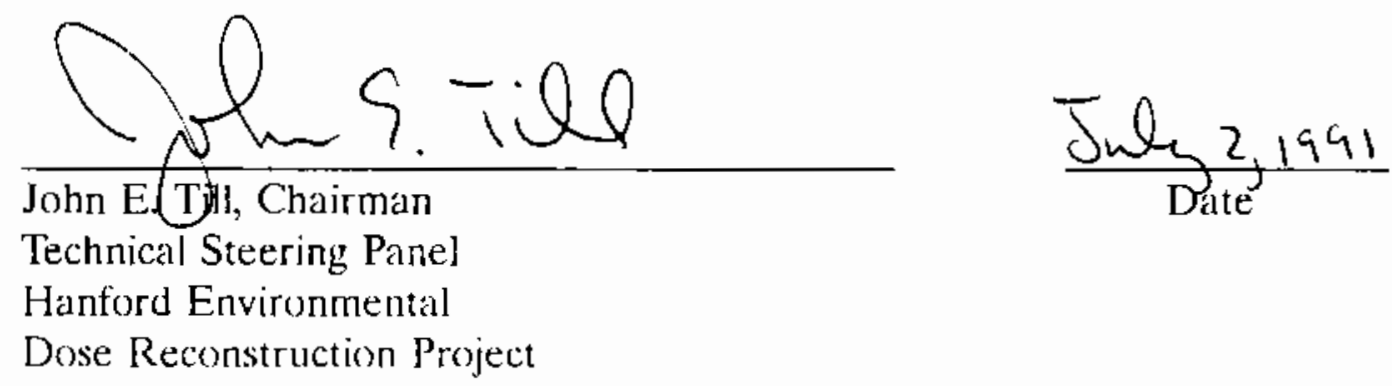


\section{PREFACE}

This report summarizes the information used in modeling atmospheric transport and diffusion for Phase $I$ of the Hanford Environmental Dose Reconstruction (HEDR) Project. It also lists the results of the atmospheric transport calculations that were provided for use in dose estimation. The report does not contain a description of the atmospheric model or an analysis of the results of the atmospheric calculations.

Atmospheric transport and diffusion calculations were made with a Lagrangian trajectory, Gaussian puff model. Rationale leading to selection of this modeling approach is discussed in another document (Ramsdell 1991). At the time that this report was written, it was not the intent to document the MESOILT2 code because it was an interim code. However, after this report was written, it became clear that documentation of MESOILT2 was essentia7. MESOILT2 is described in detail in a separate report (Ramsdell and Burk 1991). As a result, details of the model description were deleted as this report was revised for publication.

The atmospheric transport and diffusion calculations that are the subject of this report were made in late June and early July 19B9. They were made using the best meteorological data available at that time. The results of the atmospheric model calculations were electronically transferred to other HEDR staff for use in calculations leading to the Phase I dose estimates that were published in July 1990.

The primary objective of the Phase I calculations was to demonstrate that sufficient data existed and that models could be developed to permit reconstruction of doses resulting from operations at the Hanford site and that the calculations were practical. This objective was achieved. However, analysis of the results of the dose calculations indicated a need to restructure the overall dose calculation procedure. The interface between the atmospheric model and the dose model is one of the areas that needed restructuring. The treatment of uncertainty in the atmospheric model is another. Finally, several changes to MESOILI2 are required to enhance the technical credibility of the model. 
The atmospheric model is undergoing a significant revision at this time. The model revisions will address many of the concerns expressed by the reviewers of this report. When the model revisions are complete, the model will be fully documented and model sensitivity and uncertainty will be evaluated. The model revision and documentation are scheduled to be completed in 1991; model sensitivity and uncertainty studies will be conducted in the following years.

The appendix to this document is a record of ISP comments and Battelle's responses; the TSP has reviewed and approved these responses to their comments. Comment numbers appear in the left margin next to the paragraph in which the corresponding comment was addressed. Changed text is shown in italics. In addition to responses to TSP comments, some text was changed to correct errors or to provide clarification. 


\section{SUMMARY}

The Hanford Environmental Dose Reconstruction (HEDR) Project has been established to evaluate the doses people may have received from radionuclides released to the environment from operations that began at the Hanford Site in 1944. The project is being conducted by the Pacific Northwest Laboratory (PNL), operated by Battelle Memorial Institute, under the direction of an independent Technical Steering Panel.

The initial phase of the project is a demonstration of the feasibility of the technical approach recomnended by PNL. This approach involves the use of computer models and environmental monitoring data to estimate concentrations of radionuclides in the atmosphere and on the ground as a function of location and time.

A Lagrangian-trajectory, Gaussian puff atmospheric transport and diffusion model was selected for use in Phase $I$ of the HEDR Project. The model, MESOILT2, and a post-processing code, PHASE1I, used to estimate averages and uncertainty of predicted air concentrations and surface contamination are described in detail in a separate report (Ramsdell 1991). This report describes the input to the model for Phase I calculations and lists the results of the atmospheric model calculations that were used in subsequent dose calculations.

Meteorological data for 1983 through 1987 were used as model input because data for 1944 through 1947 were not ready for use at the time the atmospheric model calculations were made. Transport and diffusion calculations were made for all 60 months assuming that the same amount of iodine131 was released each month. The results of the monthly calculations were averaged to obtain typical dispersion factors for each census division for each calendar month (i.e., January, february, etc.). Uncertainty in the dispersion factors was estimated on the basis of spatial variability within the census divisions, the year-to-year variations for each month, and an estimate of the uncertainty of the model calculations for each month based on comparison with measured data. 
Specific dispersion factors were calculated for monthly time-integrated air concentrations, monthly-average deposition rates, and month-end surface contamination. These dispersion factors, which are listed in tables in the report, were passed to others for use in dose calculations. They calculated time-integrated air concentrations, deposition rates, and surface contamination for December 1944 through December 1947 for the dose estimated by multiplying the appropriate dispersion factor by the amount of iodine-131 released during the month.

Meteorological data for the period of release will be used in future calculations as such data become available. A set of calculations was made after July 1989 using 1944 through 1947 meteorological data. Figure 2.7 of the HEDR Air Pathway Report (PNL 1991) compares iodine-13I concentrations on sagebrush in Benton and Franklin county census divisions calculated using the 1983-1987 and 1944-1947 meteorological data sets. The comparison does not indicate that use of the 1983-1987 data resulted in gross errors in dose estimates. 


\section{CONTENTS}

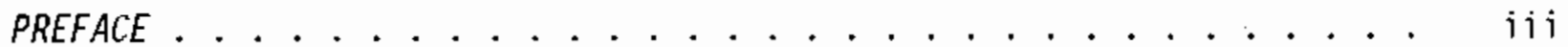

SUMMARY . . . . . . . . . . . . . . . . . . . . . . . . V

INTRODUCTION . . . . . . . . . . . . . . . . . . . . . . . . . . 1

ATMOSPHERIC TRANSPORT MODELING . . . . . . . . . . . . . . 3

ATMOSPHERIC MODELING DOMAIN . . . . . . . . . . . . . . . 3

METEOROLOGICAL DATA . . . . . . . . . . . . . . . . . . 5

RELEASE LOCATION AND SOURCE TERM . . . . . . . . . . . . . 8

MODEL COMPUTATIONS . . . . . . . . . . . . . . . . . . . 13

MODEL RESULTS . . . . . . . . . . . . . . . . . . . . . 19

REFERENCES . . . . . . . . . . . . . . . . . . . . . 4 47

APPENDIX - SUMMARY OF TSP COMMENTS AND BATTELLE RESPONSES . . . . . A. 1 


\section{FIGURES}

l Phase I Atmospheric Modeling Domain . . . . . . . . . . . . 5

2 Sources of Meteorological Data for Phase I . . . . . . . . . . 8

3 HEDR Census Divisions . . . . . . . . . . . . . . . . 14

\section{TABLES}

1 Meteorological Stations Providing the 1983-1987 Data Used in the HEDR Phase I Study . . . . . . . . . . . . . . . . . . 9

2 MESOILT2 Grid Node Associated with HEDR Census Divisions . . . . 15

3 Computational Results for January . . . . . . . . . . . . . 22

4 Computational Results for February . . . . . . . . . . . . . 24

5 Computational Results for March . . . . . . . . . . . . . 26

6 Computational Results for April . . . . . . . . . . . . 28

7 Computational Results for May . . . . . . . . . . . . 30

8 Computational Results for June . . . . . . . . . . . . . 32

9 Computational Results for July . . . . . . . . . . . . . 34

10 Computational Results for August . . . . . . . . . . . . 36

11 Computational Results for September . . . . . . . . . . . 38

12 Computational Results for October . . . . . . . . . . . . 40

13 Computational Results for November . . . . . . . . . . . . 42

14 Computational Results for December . . . . . . . . . . . . . 44 


\section{INTRODUCTION}

The Hanford Environmental Dose Reconstruction (HEOR) Project has been established to evaluate the doses people may have received from radionuclides released to the environment from operations which began at the Hanford Site in 1944. The project is being conducted by the Pacific Northwest Laboratory, operated by Battelle Memorial Institute, under the direction of an independent Technical Steering Panel. At the Technical Steering Panel's direction, the Project is being conducted in phases. The first phase is a demonstration of the feasibility of the technical approach. This approach involves the use of both computer models and environmental monitoring data to estimate concentrations of radionuclides in the atmosphere and on the ground as a function of location and time. For Phase I, the dose reconstruction domain is Benton County, Washington and the nine adjacent counties in Washington and Oregon. December 1944 through 1947 was selected as the period of interest for Phase I for investigating atmospheric releases.

A Lagrangian-trajectory, Gaussian puff atmospheric transport and diffusion model was selected for use in Phase $I$ of the HEDR Project. The model, MESOILT2, and a post-processing code, PHASEII, used to estimate averages and uncertainty of predicted air concentrations and surface contamination are described in detail in a separate report (Ramsdell 199I). This report describes the input to the model for Phase $I$ calculations and 7 ists the results of the atmospheric model calculations that were used in subsequent dose calculations.

Meteorological data for 1983 through 1987 were used as model input because data for 1944 through 1947 were not ready for use at the time the atmospheric model calculations were made. Transport and diffusion calculations were made for all 60 months assuming that the same amount of iodine131 was released each month. The results of the monthly calculations were averaged to obtain typical dispersion factors for each census division for each calendar month (i.e., January, February, etc.). Uncertainty in the dispersion factors was estimated on the basis of spatial variability within 
the census divisions, the year-to-year variations for each month, and an estimate of the uncertainty of the model calculations for each month based on comparison with measured data.

Specific dispersion factors were calculated for monthly time-integrated air concentrations, monthly-average deposition rates, and month-end surface contamination. These dispersion factors, which are listed in tables in the report, were passed to others for use in dose calculations. They calculated time-integrated air concentrations, deposition rates, and surface contamination for December 1944 through December 1947 for the dose estimated by multiplying the appropriate dispersion factor by the amount of iodine-131 released during the month.

Meteorological data for the period of release will be used in future calculations as such data become available. A set of calculations was made after.July 1989 using 1944 through 1947 meteorological data. Figure 2.7 of the HEDR Air Pathway Report (PNL 1991) compares iodine-131 concentrations on sagebrush in Benton and Franklin county census divisions calculated using the 1983-1987 and 1944-1947 meteorological data sets. The comparison does not indicate that use of the 1983-1987 data resulted in gross errors in dose estimates.

The work described in this report was conducted in accordance with the requirements of ANSI/AMSE NQA-1 1986 Edition, Quality Assurance Program Requirements for Nuclear Facilities, as interpreted by PNL's Quality Assurance (QA) program. All procedures used to support this report were appropriately written and controlled in accordance with PNL QA program requirements.

Records that support the data in this report were created and stored in accordance with applicable HEDR record control procedures.

Following completion of development, MESOILT2 was placed under configuration control and subjected to computational tests outlined in MESOILT2 Software Testing PIan. The software tests covered data input and output, as well as numerical calculations. The results of the tests were examined by the programmer and an independent reviewer. They indicated that the program was operating as intended and that the calculations were correct. 


\section{ATMOSPHERIC TRANSPORT MODELING}

The HEDR Phase I atmospheric and diffusion calculations were made with MESOILT2 (Ramsdell and Burk 1991). The model was developed for Phase I by modifying the MESOI model (Ramsdell et a1. 1983) and including some of the features of the MESORAD model (Scherpelz et al. 1986, Ramsdell et a1. 1988). MESOILT2 is a Lagrangian puff model that simulates transport and diffusion of a continuous plume by dividing the plume into discrete increments, which are referred to as puffs.

Puffs are generated every 15 minutes. They move with the wind and grow larger in response to wind turbulence. Puffs remain active and are tracked until they leave the model domain unless their mass is depleted by deposition or they merge with another puff. When the radi $i$ of two consecutive puffs become approximately equal to one half the separation between them, the puffs are merged and tracked as a single puff (Ramsdell et al. 1983).

As the puffs move, material is deposited on the ground when the puff comes in contact with the surface (dry deposition). MESOILT2 is also capable of simulating deposition when there is precipitation (wet deposition). However, this feature was not used in Phase $I$ calculations. Once material is deposited on the ground it is removed from the puff, and is tracked as surface contamination. MESOILT2 calculates decay in the atmosphere and on surfaces for radionuclides that have half-lives of less than 1 year. Decay is calculated for jodine-131, which is the radionuclide of primary interest in Phase $I$.

\section{ATMOSPHERIC MODELING DOMAIN}

A square atmospheric transport modeling domain, $160 \mathrm{mi}$ (256 km) on a side centered at $46^{\circ} 37^{\prime} 30^{\prime \prime} \mathrm{N}$ latitude, $119^{\circ} 00^{\prime} \mathrm{W}$ longitude was selected for Phase I. Physically, the center of the domain is about $25 \mathrm{mi}(40 \mathrm{~km})$ east of the 200 Areas of the Hanford Site. Most of the atmospheric releases of iodine-131 occurred in the 200-East and 200-West Areas. The domain extends from the Cascade Mountains east to approximately the Washington-Idaho border. The southwest corner of the domain is near The Dalles, Oregon, and the northeast corner is near Spokane, Washington. 
Air concentrations and surface contamination were computed at 931 nodes within the domain. The spacing between nodes is about $5 \mathrm{mi}(8 \mathrm{~km})$. This separation was established on the basis of the desired domain size and the structure of the model from which MESOILT2 was developed. The spacing proved adequate to define average time-integrated air concentrations and surface con-. tamination for the census divisions for which doses were calculated. However, analysis of the Phase $I$ results indicates that computing doses from the census division data must be reevaluated.

Positions in the model domain are identified by Cartesian coordinates. The lower left corner of the domain has coordinates 1,1; the lower right corner has coordinates 31,1; and the upper right corner has coordinates 31,31 .

The atmospheric modeling domain covers the 10-county Phase I study area except for the western portions of Kittitas, Yakima, and Klickitat Counties, the northern portion of Grant County, and the southern portions of Morrow and Umatilla Counties. The relationship between the atmospheric modeling domain and the 10-county area is shown in Figure 1 . County names are shown in the figure in light print, and selected population centers are shown in bold print.

The MESOILT2 domain was off set from the 10-county area for three reasons. The first reason is that the prevailing wind patterns at Hanford have westerly components, which carry material released at Hanford to the east. Thus, offsetting the domain to the east increased the proportion of the domain that is downwind of Hanford in the prevailing atmospheric transport directions. The second reason is that winds with easterly components, which carry material to the west, are infrequent, are generally light, and do not persist for long periods. The third reason is that the terrain to the west of the MESOILT2 domain is mountainous. Concentration and deposition estimates for the western boundary of the MESOILT2 domain were assumed to be conservative estimates of the concentrations and deposition in the portion of the 10-county region to the west of the model domain. 


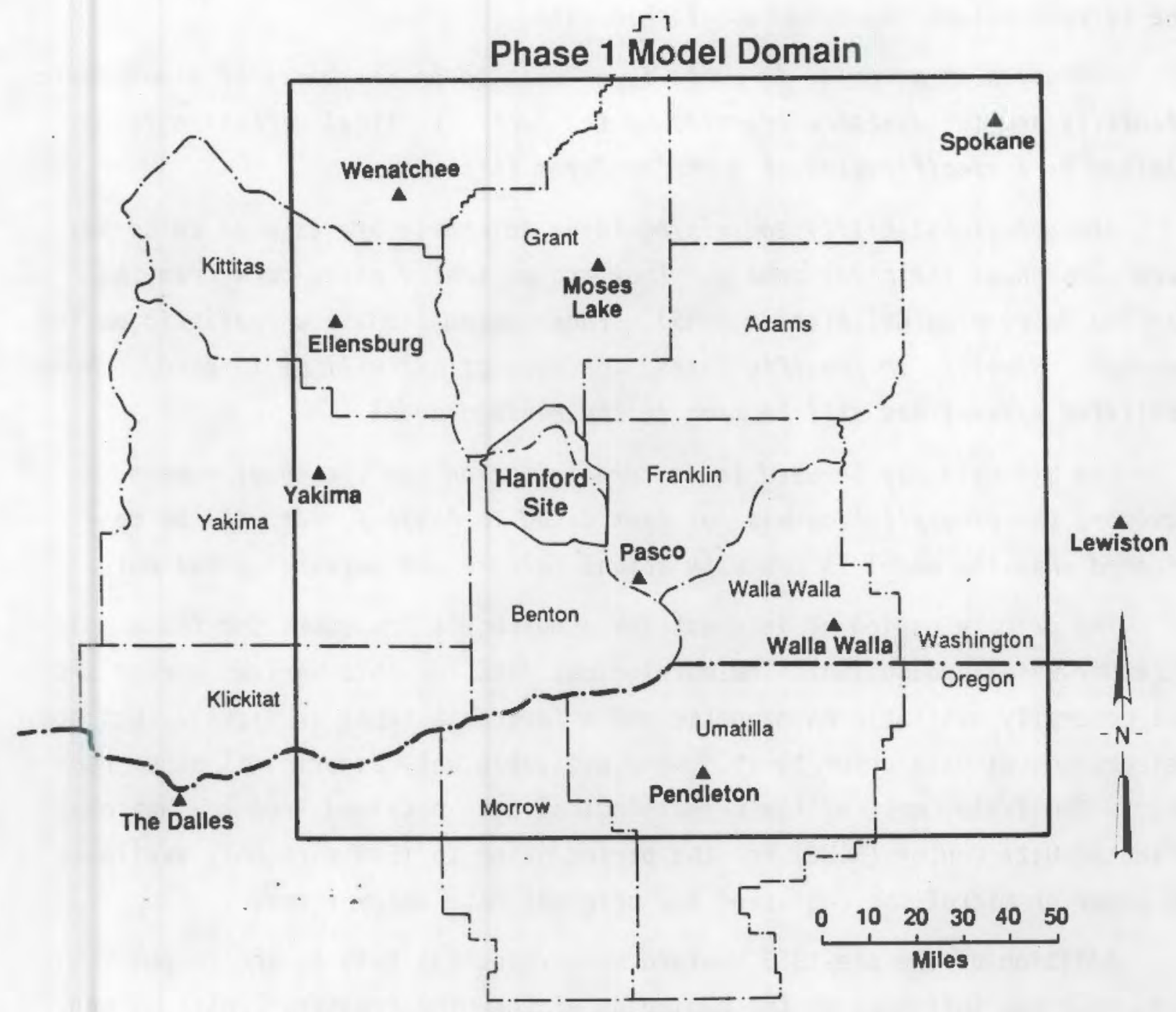

FIGURE 1. Phase I Atmospheric Modeling Domain

\section{METEOROLOGICAL DATA}

Observed meteorological data are used in MESOILT2 to make transport and diffusion calculations. Transport is based on wind data, and diffusion is based on wind data, atmospheric stability, and mixing-layer thickness.

Wind data from all measurement locations are used to estimate the wind fields that are used to compute puff movements. An initial estimate of the wind field is made from the observed wind data using $1 / r^{2}$ interpolation. This initial wind field is adjusted to account for expected terrain effects based 
on the changes in elevation of the topography and steepness of the slope of the terrain determined from topographic data.

Diffusion of material in puffs is calculated on the basis of atmospheric stability and the distance traveled by the puff. Vertical diffusion is limited by a specification of a mixing-layer thickness.

Atmospheric stability and mixing-layer thickness are assumed to be uniform throughout the model domain. They are estimated using data from the Hanford Meteorological Station (HMS). These assumptions are realistic on the average. However, in specific cases, the assumptions will be in error. More realistic assumptions will be made in the revised model.

The HMS data may be used to set precipitation for the model domain. However, the precipitation was not considered in Phase I. It will be considered when the model is run with actual release and meteorological data.

The primary period of interest for atmospheric transport for Phase I is December 1944 through 1947. Meteorological data for this earlier period are not generally available on magnetic media (magnetic tapes or disks). Hanford meteorological data prior to 1955 were available only as original paper records. Similarly, most of the meteorological data obtained from the National Climatic Data Center (NCDC) for the period prior to 1948 were only available as paper or microfiche copies of the original data entry forms.

Addition of the pre-1955 Hanford meteorological data to the computer data base was initiated at the beginning of the HEDR Project. Entry of meteorological data through 1949 has been completed. Meteorological data for other stations for 1944 through 1947 were ordered from the NCDC for use in the Phase I study. However, these data were not received in time to be used in the Phase I atmospheric transport calculations.

When it became obvious that the 1944 through 1947 meteorological data would not be ready for use in the Phase I calculations, two alternative approaches for estimating iodine-131 air concentrations and surface contamination were considered. The two alternatives considered were 1) to use the 1944 through 1947 data for Hanford and a straight-line Gaussian model and 2) to use data from another period and MESOILT2. While the first alternative 
would use data from the period of interest, the second alternative was selected because it provided a better test of the overall atmospheric modeling process.

The Phase I model calculations were made with meteorological data for 1983 through 1987 because more meteorological data are readily available for this period than are available for other periods. The available data include routine observations for 12 locations in eastern Washington, northeastern Oregon and northern Idaho in addition to data from the Hanford Telemetry System, which provides wind measurements at more than 20 locations on and adjacent to the Hanford Site. Figure 2 shows the locations of the meteorological stations providing data for the Phase I atmospheric transport calculations. Primary meteorological stations are shown by triangles in the figure and the stations in the Hanford Telemetry System are shown by dots. The station names, abbreviations, locations, and MESOILT2 grid coordinates are listed in Table 1.

Entry of available wind data for 1944 through 1947 was completed in late fall of 1989. MESOILT2 was rerun using these data. Figure 2.7 of the Air Pathway Report (PNL 1991) shows a comparison of iodine-131 concentrations on sagebrush in Benton and Franklin counties calculated using the 1944-1947 and 1983-1987 meteorological data. The results of the two sets of calculations are very similar. This similarity is likely the result of assuming a continuous, uniform release of iodine-131 for each month.

MESOILT2 can use meteorological data observed at either hourly or 3-hour intervals. If hourly data are used, the meteorological conditions are assumed to persist for the entire hour; there is no temporal interpolation within the hour. If, however, 3-hourly meteorological data are used, winds, atmospheric stability, and precipitation are updated each hour using linear interpolation between the meteorological observations. The Phase I model runs were made using 3-hourly meteorological data. Hourly data were available for most of the locations, but not all of the data were on magnetic media. Therefore, a decision was made to use 3-hour data in the calculations. Those data not already available on magnetic media were manually entered into the HEDR meteorological data base for use in the Phase I calculations, and all data were checked for reasonableness. 


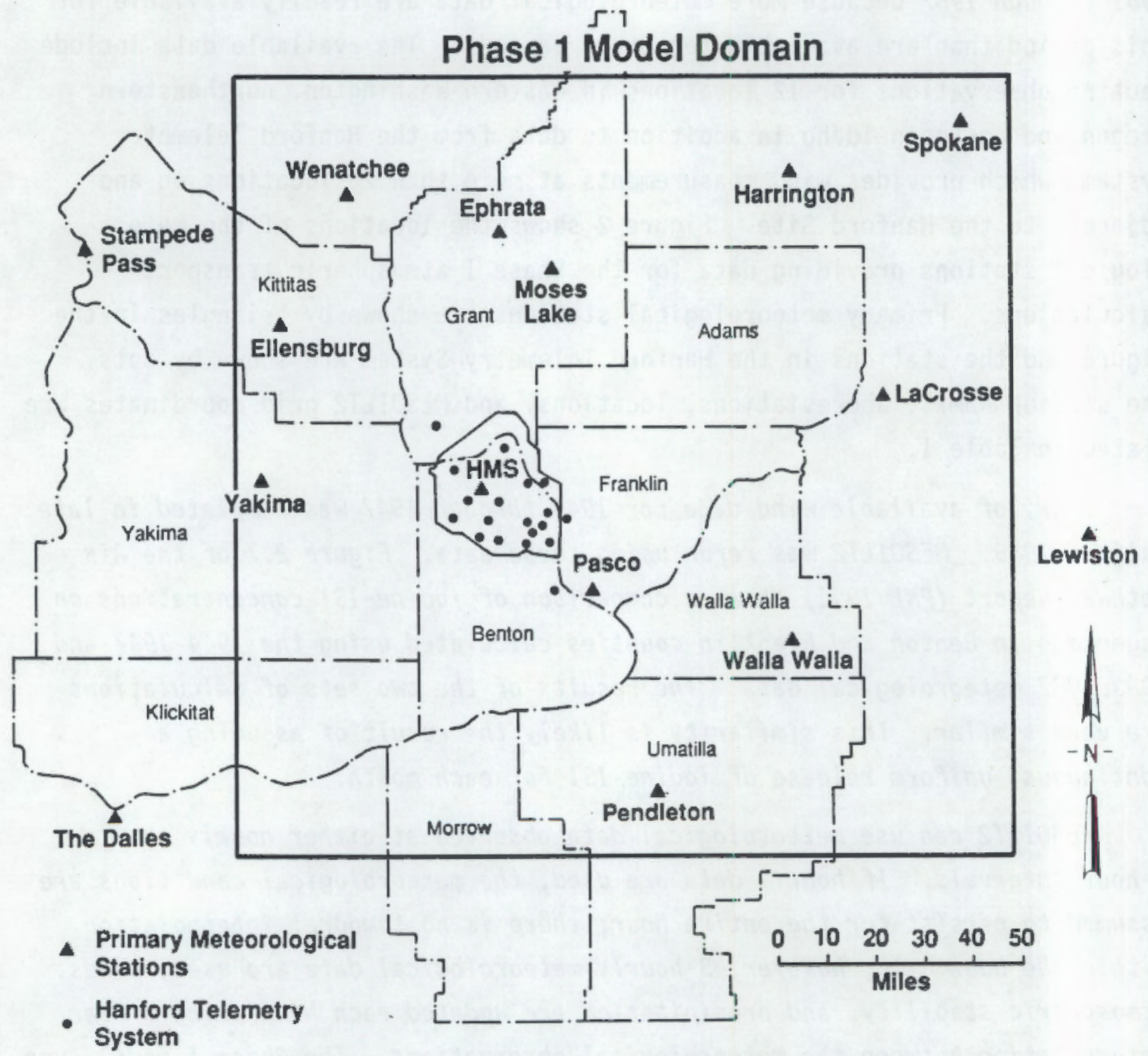

FIGURE 2. Sources of Meteorological Data for Phase I

\section{RELEASE LOCATION AND SOURCE TERM}

Phase I atmospheric transport modeling is primarily concerned with iodine-131 releases that took place from December 1944 through 1947 . These releases were associated with the fuels separation processing in $B$ and $T$ 
IABLE 1. Meteorological Stations Providing the 1983-1987 Data Used in the HEDR Phase I Study

Latitude Longitude Elev. MESOILT2 Grid

\begin{tabular}{|c|c|c|c|c|c|c|c|c|c|c|}
\hline Station Name & Abbrev. & Deg & Min & $\underline{\operatorname{Sec}}$ & Deq & Min & Sec & (m) & $\underline{X}$ & $Y$ \\
\hline PROSSER BARRICADE & PROS & 46 & 23 & 31 & 119 & 24 & 40 & 146 & 12.08 & 12.76 \\
\hline EMERG. OPS. CENTER & $\mathrm{EOC}$ & 46 & 23 & 33 & 119 & 32 & 10 & 378 & 10.88 & 12.77 \\
\hline ARMY LOOP ROAD & ARMY & 46 & 29 & 19 & 119 & 32 & 53 & 172 & 10.77 & 14.10 \\
\hline RATTLESNAKE SPRINGS & RSPG & 46 & 30 & 22 & 119 & 41 & 56 & 207 & 9.33 & 14.35 \\
\hline EDNA & EDNA & 46 & 35 & 15 & 119 & 23 & 50 & 122 & 12.21 & 15.48 \\
\hline 200 EAST AREA & $200 \mathrm{E}$ & 46 & 33 & 23 & 119 & 31 & 14 & 204 & 11.03 & 15.05 \\
\hline 200 WEST AREA & $200 \mathrm{~W}$ & 46 & 32 & 35 & 119 & 39 & 41 & 201 & 9.69 & 14.86 \\
\hline WAHLUKE SLOPE & WAHL & 46 & 44 & 7 & 119 & 50 & & 258 & 8.02 & 17.53 \\
\hline FAST FLUX TEST FAC. & FFTF & 46 & 25 & 49 & 119 & 21 & & 168 & 12.58 & 3.29 \\
\hline YAKIMA BARRICADE & YAKB & 46 & 34 & 41 & 119 & 43 & & 242 & 9.08 & 5.35 \\
\hline 300 AREA & $300 A$ & 46 & 21 & 50 & 119 & 17 & & 122 & 13.27 & 12.37 \\
\hline WYE BARRICADE & WYEB & 46 & 28 & 56 & 119 & 23 & 34 & 168 & 12.25 & 14.02 \\
\hline 100 NORTH AREA & $100 N$ & 46 & 41 & 16 & 119 & 32 & 58 & 146 & 10.76 & 16.87 \\
\hline WASH PUB PWR SUP & WPPS & 46 & 28 & 12 & 119 & 20 & 34 & 137 & 12.73 & 13.85 \\
\hline FRANKLIN COUNTY & FRNK & 46 & 25 & 3 & 119 & 14 & 12 & 268 & 13.74 & 13 \\
\hline GABLE MOUNTAIN & GABL & 46 & 35 & 53 & 119 & 27 & 36 & 331 & 11.61 & 15.63 \\
\hline $\begin{array}{l}\text { RINGOLD } \\
\text { R }\end{array}$ & RING & 46 & 32 & 42 & 119 & 14 & 13 & 189 & 13.74 & 14 \\
\hline RICHLAND & $\mathrm{RICH}$ & 46 & 18 & 4 & 119 & 18 & 1 & 119 & 13.13 & 11. \\
\hline SAGE HILL & SAGE & 46 & 39 & 21 & 119 & 17 & 58 & 300 & 13.14 & 16.43 \\
\hline RATTLESNAKE MTN & RMTN & 46 & 23 & 40 & 119 & 35 & 37 & 1087 & 10.33 & 12.80 \\
\hline HANFORD MET. STA. & HMS & 46 & 33 & 47 & 119 & 35 & 54 & 222 & 10.29 & 5.14 \\
\hline PASCO & PASC & 46 & 15 & 48 & 119 & 6 & 18 & 122 & 15.00 & 0.97 \\
\hline 100 F AREA & $100 \mathrm{~F}$ & 46 & 38 & 6 & 119 & 27 & 4 & 123 & 11.69 & 16.14 \\
\hline GABLE MTN WEST & GABW & 46 & 36 & 35 & 119 & 33 & 23 & 305 & 10.69 & 15.79 \\
\hline YAKIMA & YKM & 46 & 34 & 0 & 120 & 32 & 0 & 326 & 1.37 & 15.19 \\
\hline PENDLETON & PDT & 45 & 41 & 0 & 118 & 51 & 0 & 459 & 17.43 & 2.91 \\
\hline STAMPEDE PASS & SMP & 47 & 17 & 0 & 121 & 20 & 0 & 1209 & -6.27 & 25.15 \\
\hline LEWISTON & LWS & 46 & 23 & 0 & 117 & 1 & 0 & 439 & 34.93 & 12.64 \\
\hline SPOKANE & SKA & 47 & 38 & 0 & 117 & 32 & 0 & 718 & 30.00 & 30.01 \\
\hline WALLA WALLA & ALW & 46 & 6 & 0 & 118 & 17 & 0 & 36 & 22.84 & 8.70 \\
\hline MOSES LAKE & MWH & 47 & 12 & 0 & 119 & 19 & 0 & 376 & 12.98 & 23.99 \\
\hline THE DALLES & DLS & 45 & 37 & 0 & 121 & 9 & 0 & 72 & -4.52 & 1.99 \\
\hline WENATCHEE & EAT & 47 & 24 & 0 & 120 & 12 & 0 & 24 & 4.55 & 26.77 \\
\hline EPHRATA & $\mathrm{EPH}$ & 47 & 18 & 0 & 119 & 32 & 0 & 38 & 10.91 & 25.38 \\
\hline OMAK & OMK & 48 & 26 & 0 & 119 & 32 & 0 & 26 & 10.91 & 41.13 \\
\hline COLVILLE & CVL & 48 & 32 & 0 & 117 & 53 & 0 & 575 & 26.66 & 42.52 \\
\hline
\end{tabular}

plants, which are in the 200-East and 200-West Areas, respectively. Prior to 1948, iodine-131 was released from these plants directly to the atmosphere with gases produced during dissolution of the reactor fuels (Ballinger and Hall 1991). 
Off gases from B and $T$ plants were released to the atmosphere through $200-\mathrm{ft}(60-\mathrm{m})$ stacks that have a $5-\mathrm{ft}(1.52-\mathrm{m})$ inside diameter. The nominal flow in the stacks is $25,000 \mathrm{ft}^{3} / \mathrm{min}\left(11.7 \mathrm{~m}^{3} / \mathrm{s}\right)$ (Smith 1950). Ballinger and Hall (1991, Figure 4.1) show that off gases were combined with ventilation air. With exhaust $f$ ans operating, the stack flow could reach $65,000 \mathrm{ft}^{3} / \mathrm{min}$ $\left(30.7 \mathrm{~m}^{3} / \mathrm{s}\right)$.

$B$ and T Plant stacks are separated by a distance of about $5 \mathrm{mi}(8 \mathrm{~km})$. However, for the purpose of demonstration of the technical approach in Phase I, the releases were assumed to take place from a single, imaginary stack located midway between the two stacks. A height of $200 \mathrm{ft}(61 \mathrm{~m})$ and a flow of $20,000 \mathrm{ft}^{3} / \mathrm{min}\left(9.4 \mathrm{~m}^{3} / \mathrm{s}\right)$ were assumed. No attempt was made to estimate plume rise during the Phase I calculations. However, the stack flow was used to set the initial magnitudes of the diffusion coefficients to avoid an atmospheric concentration of iodine-131 that exceeded the concentration in the stack.

MESOILT2 has the capability to vary the release rate hour by hour. In Phase I this capability was not used for two reasons. The first, and overriding, reason is that sufficient data on release times and rates were not available. The second reason is that the meteorological data for the release period were not available. Release data currently available include dates and generally permit estimation of release times to within one of two shifts, and meteorological data for the 1944-1947 period are now available. Consequent7y, future calculations will be made using the actual release and meteorological data.

The only viable alternative to using the actual release data was to assume a uniform, constant release rate for each month. This assumption had two probable effects. It likely resulted in a contamination pattern that was more wide spread than occurred. It is also likely that this assumption somewhat reduced the magnitude of the contamination in the prevailing downwind direction. However, the comparison of predicted iodine-131 concentrations in sagebrush with observed values shown in the MESOILT2 documentation does not indicate that this postulated reduction in contamination is significant. 
The release rate assumed for the Phase I study was 1000 curies (Ci) of iodine-131 per month. This source term was entered as an hourly release equal to $1000 \mathrm{Ci}$ divided by the number of hours per month. As a result, the source term used in the computation varied from month to month. This release rate was selected to keep atmospheric iodine-131 concentrations above the MESOILT2 low concentration threshold. During post-processing, all monthly atmospheric concentrations and surface contamination values were normalized by dividing by $1000 \mathrm{Ci}$. Normalization permits users of the MESOILT2 output to estimate concentrations and surface contamination for specific months by multiplying the normalized values by the amount of iodine-131 released during the month. 



\section{MODEL COMPUTATIONS}

MESOILT2 performs computations on a month-by-month basis. At the end of each month, a set of computer files is written that contains the results of the model calculations. However, there are a number of active puffs remaining in the domain at the end of each month. These puffs contribute to the time-integrated air concentration and surface contamination in the following month. Consequently, the information on these puffs must be carried forward into the calculations for the next month. MESOILT2 does this by creating a file at the end of the month that contains characteristics of all puffs that are still on the grid. This file, which includes puff numbers, positions, dimensions and masses, becomes input to the computations for the next month.

The MESOILT2 computations are made at nodes on a $31 \times 31$ Cartesian grid although dose estimates are to be made for the HEDR census divisions shown in Figure 3. A post-processor computer program, PHASE1I, converts the MESOILT2 output to the monthly time-integrated atmospheric concentrations, deposition rates, and month-end surface contaminations for the HEDR census divisions. The PHASEII program is listed in Appendix $B$ of the MESOILT2 documentation (Ramsdell and Burk 1991).

Conversion of the node values to census division averages involves averaging the values at nodes in and near the census divisions. Table 2 lists the nodes in the model domain that are associated with each census division. The process also involves averaging the values for each of the five years. As a result, the census division values are averages over both time and space. 


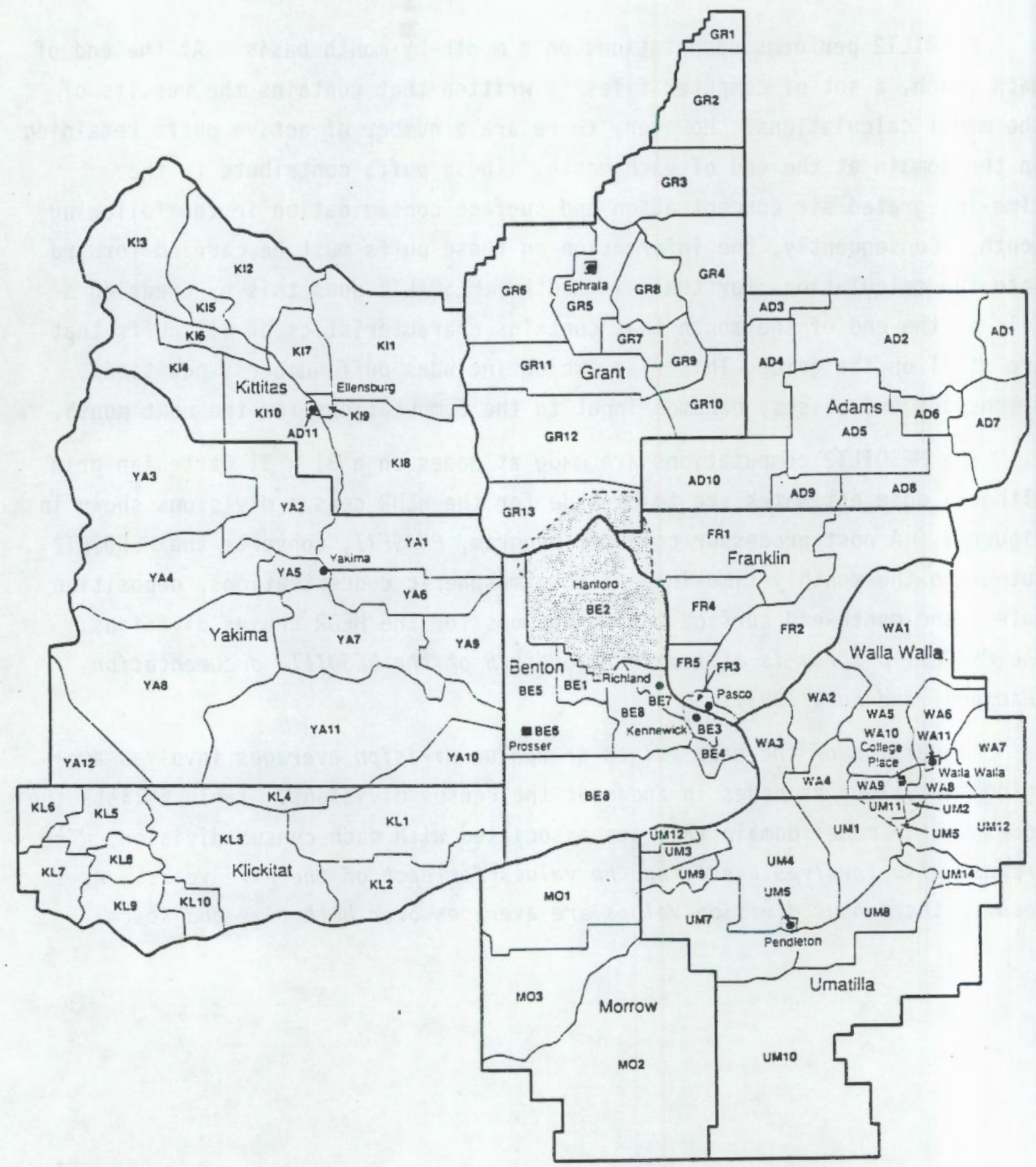

FIGURE 3. HEDR Census Divisions 
TABLE 2. MESOILT2 Grid Node Associated with HEDR Census Divisions. The MESOILT2 nodes are given by their coordinates. The first number in each pair is the x-coordinate (east-west), and the second is the $y$-coordinate (north-south).

\begin{tabular}{|c|c|c|c|c|c|c|c|c|c|}
\hline $\begin{array}{l}\text { Census } \\
\text { Div. }\end{array}$ & & & & MESS & T? & & & & \\
\hline $\begin{array}{l}A D 1 \\
A D 2\end{array}$ & $\begin{array}{l}25,25 \\
20,25 \\
22,24 \\
24,23\end{array}$ & $\begin{array}{l}26,25 \\
21,25 \\
23,24 \\
21,22\end{array}$ & $\begin{array}{l}25,24 \\
22,25 \\
24,24 \\
22,22\end{array}$ & $\begin{array}{l}26,24 \\
23,25 \\
18,23 \\
23,22\end{array}$ & $\begin{array}{l}25,23 \\
24,25 \\
19,23 \\
24,22\end{array}$ & $\begin{array}{l}26,23 \\
18,24 \\
20,23\end{array}$ & $\begin{array}{l}25,22 \\
19,24 \\
21,23\end{array}$ & $\begin{array}{l}26,22 \\
20,24 \\
22,23\end{array}$ & $\begin{array}{l}21,24 \\
23,23\end{array}$ \\
\hline AD3 & 17,25 & 18,25 & 19,25 & 20,25 & 17,24 & 18,24 & & & \\
\hline & 17,24 & 18,24 & 17,23 & 18,23 & 17,22 & & 17,21 & 18,21 & 17,20 \\
\hline AD5 & $\begin{array}{l}18,22 \\
19,20\end{array}$ & $\begin{array}{l}19,22 \\
20,20\end{array}$ & $\begin{array}{l}20,22 \\
21,20\end{array}$ & $\begin{array}{l}21,22 \\
18,19\end{array}$ & $\begin{array}{l}18,21 \\
19,19\end{array}$ & $\begin{array}{l}19,21 \\
20,19\end{array}$ & $\begin{array}{l}20,21 \\
21,19\end{array}$ & & 8,20 \\
\hline AD6 & 21,21 & 22,21 & 23,21 & 24,21 & 22,20 & 23,20 & & & \\
\hline AD7 & $\begin{array}{l}25,22 \\
25,19\end{array}$ & $\begin{array}{l}26,22 \\
26,19\end{array}$ & $\begin{array}{l}24,21 \\
25,18\end{array}$ & 25,21 & 26,21 & 24,20 & 25,20 & 26,20 & 24,19 \\
\hline AD8 & $\begin{array}{l}22,20 \\
24,18\end{array}$ & 23,20 & 22,19 & 23,19 & 24,19 & 20,18 & 21,18 & 22,18 & 23,18 \\
\hline AD9 & $\begin{array}{l}17,19 \\
19,17\end{array}$ & 18,19 & 19,19 & 17,18 & 18,18 & 19,18 & 20,18 & 17,17 & 18,17 \\
\hline AD10 & $\begin{array}{l}13,20 \\
17,19\end{array}$ & $\begin{array}{l}14,20 \\
13,18\end{array}$ & $\begin{array}{l}15,20 \\
14,18\end{array}$ & $\begin{array}{l}16,20 \\
15,18\end{array}$ & $\begin{array}{l}17,20 \\
16,18\end{array}$ & $\begin{array}{l}13,19 \\
17,18\end{array}$ & 14,19 & 15,19 & 16,19 \\
\hline BE 1 & 10,13 & 10,12 & 11,12 & 10,11 & 11,11 & & & & \\
\hline & $\begin{array}{r}9,17 \\
9,15 \\
13,14\end{array}$ & $\begin{array}{l}10,17 \\
10,15 \\
10\end{array}$ & $\begin{array}{l}11,17 \\
11,15\end{array}$ & $\begin{array}{l}12,17 \\
12,15 \\
12\end{array}$ & $\begin{array}{r}8,16 \\
13,15\end{array}$ & $\begin{array}{l}9,16 \\
9,14\end{array}$ & $\begin{array}{l}10,16 \\
10,14\end{array}$ & $\begin{array}{l}11,16 \\
11,14\end{array}$ & $\begin{array}{l}12,16 \\
12,14\end{array}$ \\
\hline BE3 & $\begin{array}{l}15,14 \\
14,11\end{array}$ & $\begin{array}{l}10,13 \\
14,10\end{array}$ & $\begin{array}{l}11,13 \\
15,10\end{array}$ & $\begin{array}{l}12,13 \\
15,9\end{array}$ & & & & & \\
\hline $\begin{array}{l}\text { BE4 } \\
\text { BE5 }\end{array}$ & $\begin{array}{r}14,10 \\
8,16\end{array}$ & $\begin{array}{r}15,10 \\
8,15\end{array}$ & $\begin{array}{r}16,10 \\
8,14\end{array}$ & $\begin{array}{r}14,9 \\
8,13\end{array}$ & $\begin{array}{r}15,9 \\
9,13\end{array}$ & $\begin{array}{r}16,9 \\
8,12\end{array}$ & $\begin{array}{r}15,8 \\
9,12\end{array}$ & 8,11 & \\
\hline & & 8,10 & $\begin{array}{l}9,10 \\
8,10\end{array}$ & 10,10 & 11,10 & 8,9 & 9,9 & 10,9 & 11,9 \\
\hline $\begin{array}{l}\text { BE6 } \\
\text { BE7 }\end{array}$ & $\begin{array}{r}8,11 \\
12,12 \\
0\end{array}$ & $\begin{array}{r}9,11 \\
13,12\end{array}$ & $\begin{array}{r}8,10 \\
14,12\end{array}$ & $\begin{array}{r}9,10 \\
13,11\end{array}$ & $\begin{array}{r}8,9 \\
14,11\end{array}$ & $\begin{array}{r}9,9 \\
14,10\end{array}$ & & & \\
\hline BE8 & $\begin{array}{r}8,9 \\
10,8 \\
10, \\
10,6\end{array}$ & $\begin{aligned} 9, & 9 \\
11, & 8 \\
11, & 7 \\
11, & 6\end{aligned}$ & 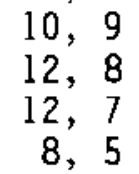 & $\begin{array}{l}11,9 \\
13,8 \\
13,7\end{array}$ & $\begin{array}{l}12,9 \\
14,8 \\
14,7\end{array}$ & $\begin{array}{l}13,9 \\
15,8 \\
15,7\end{array}$ & $\begin{array}{l}14,9 \\
16,8 \\
16,9\end{array}$ & $\begin{array}{l}8,8 \\
8,7 \\
8,6\end{array}$ & $\begin{array}{l}9,8 \\
9,7 \\
9,6\end{array}$ \\
\hline $\begin{array}{l}\text { BE9 } \\
\text { FRI }\end{array}$ & $\begin{array}{l}11,12 \\
13,17 \\
15,16 \\
18,15\end{array}$ & $\begin{array}{l}12,12 \\
14,17 \\
16,16\end{array}$ & $\begin{array}{l}11,11 \\
15,17 \\
17,16\end{array}$ & $\begin{array}{l}12,11 \\
16,17 \\
18,16\end{array}$ & $\begin{array}{l}13,11 \\
17,17 \\
13,15\end{array}$ & $\begin{array}{l}12,10 \\
18,17 \\
14,15\end{array}$ & $\begin{array}{l}13,10 \\
19,17 \\
15,15\end{array}$ & $\begin{array}{l}14,10 \\
13,16 \\
16,15\end{array}$ & $\begin{array}{l}12,9 \\
14,16 \\
17,15\end{array}$ \\
\hline FR2 & $\begin{array}{l}20,17 \\
19,15 \\
16,12\end{array}$ & $\begin{array}{l}21,17 \\
20,15 \\
17,12\end{array}$ & $\begin{array}{l}22,17 \\
17,14 \\
18,12\end{array}$ & $\begin{array}{l}23,17 \\
18,14 \\
16,11\end{array}$ & $\begin{array}{l}19,16 \\
19,14 \\
17,11\end{array}$ & $\begin{array}{l}20,16 \\
16,13\end{array}$ & $\begin{array}{l}21,16 \\
17,13\end{array}$ & $\begin{array}{l}22,16 \\
18,13\end{array}$ & \\
\hline
\end{tabular}


IABLE 2. (contd)

\section{Census}

Div.

MESOILT2 NODES

\begin{tabular}{|c|c|c|c|c|c|c|c|c|c|}
\hline $\begin{array}{l}\text { FR3 } \\
\text { FR4 }\end{array}$ & $\begin{array}{l}13,11 \\
14,15\end{array}$ & $\begin{array}{l}14,11 \\
15,15\end{array}$ & $\begin{array}{l}15,11 \\
16,15\end{array}$ & $\begin{array}{l}14,10 \\
14,14\end{array}$ & $\begin{array}{l}15,10 \\
15,14\end{array}$ & $\begin{array}{l}16,10 \\
16,14\end{array}$ & 14,13 & 15,13 & \\
\hline FR5 & 14,13 & 15,13 & 14,12 & 15,12 & 14,11 & 15,11 & & & \\
\hline $\begin{array}{l}\text { GR2 } \\
\text { GR3 }\end{array}$ & $\begin{array}{l}13,31 \\
12,29\end{array}$ & $\begin{array}{l}14,31 \\
13,29\end{array}$ & $\begin{array}{l}15,31 \\
14,29\end{array}$ & $\begin{array}{l}16,31 \\
15,29\end{array}$ & $\begin{array}{l}13,30 \\
16,29\end{array}$ & $\begin{array}{l}14,30 \\
11,28\end{array}$ & $\begin{array}{l}15,30 \\
12,28\end{array}$ & $\begin{array}{l}16,30 \\
13,28\end{array}$ & 14,28 \\
\hline & 15,28 & 16,28 & 11,27 & 12,27 & 13,27 & 14,27 & 15,27 & 16,27 & \\
\hline GR4 & $\begin{array}{l}13,27 \\
15,25\end{array}$ & $\begin{array}{l}14,27 \\
16,25\end{array}$ & $\begin{array}{l}15,27 \\
15,24\end{array}$ & $\begin{array}{l}16,27 \\
16,24\end{array}$ & $\begin{array}{l}13,26 \\
15,23\end{array}$ & $\begin{array}{l}14,26 \\
16,23\end{array}$ & 15,26 & 16,26 & 14,25 \\
\hline GR5 & $\begin{array}{l}11,27 \\
10,23\end{array}$ & $\begin{array}{l}11,26 \\
11,23\end{array}$ & 12,26 & 10,25 & 11,25 & 12,25 & 10,24 & 11,24 & 12,24 \\
\hline GR6 & $\begin{array}{r}8,27 \\
8,25 \\
10,23\end{array}$ & $\begin{array}{l}9,27 \\
9,25\end{array}$ & $\begin{array}{l}10,27 \\
10,25\end{array}$ & $\begin{array}{l}7,26 \\
7,24\end{array}$ & $\begin{array}{l}8,26 \\
8,24\end{array}$ & $\begin{array}{l}9,26 \\
9,24\end{array}$ & $\begin{array}{r}10,26 \\
7,23\end{array}$ & $\begin{array}{r}11,26 \\
8,23\end{array}$ & $\begin{array}{l}7,25 \\
9,23\end{array}$ \\
\hline GR7 & 12,23 & 13,23 & 14,23 & 12,22 & 13,22 & 14,22 & & & \\
\hline GR8 & $\begin{array}{l}12,26 \\
13,23\end{array}$ & $\begin{array}{l}13,26 \\
14,23\end{array}$ & 14,26 & 12,25 & & 14,25 & 12,24 & 13,24 & 14,24 \\
\hline GR9 & & & 14,22 & 15,22 & & 14,21 & & & \\
\hline G & $\begin{array}{r}7,23 \\
12,22\end{array}$ & $\begin{array}{r}8,23 \\
13,22\end{array}$ & $\begin{array}{r}9,23 \\
7,21\end{array}$ & $\begin{array}{r}10,23 \\
8,21\end{array}$ & $\begin{array}{l}7,22 \\
9,21\end{array}$ & $\begin{array}{r}8,22 \\
10,21\end{array}$ & $\begin{array}{r}9,22 \\
11,21\end{array}$ & $\begin{array}{l}10,22 \\
12,21\end{array}$ & $\begin{array}{l}11,22 \\
13,21\end{array}$ \\
\hline GR12 & $\begin{array}{l}7,21 \\
7,19\end{array}$ & $\begin{array}{l}8,21 \\
8,19\end{array}$ & $\begin{array}{l}7,20 \\
9,19\end{array}$ & $\begin{array}{r}8,20 \\
10,19\end{array}$ & $\begin{array}{r}9,20 \\
11,19\end{array}$ & $\begin{array}{l}10,20 \\
12,19\end{array}$ & 11,20 & 12,20 & 13,20 \\
\hline GR13 & 7,18 & 8,18 & 9,18 & 10,18 & 11,18 & 12,18 & 7,17 & 8,17 & 9,17 \\
\hline GR14 & 10,26 & 11,26 & 12,26 & 10,25 & 11,25 & 12,25 & 10,24 & 11,24 & 12,24 \\
\hline KII & $\begin{array}{l}2,25 \\
5,23 \\
6,21\end{array}$ & $\begin{array}{l}2,24 \\
6,23\end{array}$ & $\begin{array}{l}3,24 \\
3,22\end{array}$ & $\begin{array}{l}4,24 \\
4,22\end{array}$ & $\begin{array}{l}5,24 \\
5,22\end{array}$ & $\begin{array}{l}6,24 \\
6,22\end{array}$ & $\begin{array}{l}2,23 \\
3,21\end{array}$ & $\begin{array}{l}3,23 \\
4,21\end{array}$ & $\begin{array}{l}4,23 \\
5,21\end{array}$ \\
\hline $\mathrm{KI} 2$ & 1,25 & 2,25 & 1,24 & 2,24 & & & & & \\
\hline KI7 & 1,24 & 2,24 & 1,23 & 2,23 & 1,22 & 2,22 & & & \\
\hline KI8 & $\begin{array}{l}3,21 \\
5,19\end{array}$ & $\begin{array}{l}4,21 \\
6,19\end{array}$ & $\begin{array}{l}2,20 \\
7,19\end{array}$ & $\begin{array}{l}3,20 \\
3,18\end{array}$ & $\begin{array}{l}4,20 \\
4,18\end{array}$ & $\begin{array}{l}5,20 \\
5,18\end{array}$ & $\begin{array}{l}6,20 \\
6,18\end{array}$ & $\begin{array}{l}3,19 \\
7,18\end{array}$ & 4,19 \\
\hline KI9 & 1,22 & 2,22 & 3,22 & 1,21 & 2,21 & 3,21 & 1,20 & 2,20 & 3,20 \\
\hline KI 10 & 1,21 & 1,20 & 2,20 & 1,19 & 2,19 & 2,18 & & & \\
\hline $\mathrm{KLI}$ & $\begin{array}{l}3,8 \\
5,7\end{array}$ & $\begin{array}{l}4,8 \\
6,7\end{array}$ & $\begin{array}{ll}5, & 8 \\
7, & 7\end{array}$ & $\begin{array}{ll}6, & 8 \\
1, & 6\end{array}$ & $\begin{array}{ll}7, & 8 \\
2, & 6\end{array}$ & $\begin{array}{l}1,7 \\
3,6\end{array}$ & $\begin{array}{l}2,7 \\
4,6\end{array}$ & $\begin{array}{ll}3, & 7 \\
5, & 6\end{array}$ & $\begin{array}{ll}4, & 7 \\
6, & 6\end{array}$ \\
\hline $\mathrm{KL} 2$ & & 2,5 & 3,5 & 4,5 & 5,5 & 6,5 & 7,5 & 3,4 & 4,4 \\
\hline KL3 & 1,6 & 2,6 & 1,5 & 2,5 & 1,4 & 2,4 & & & \\
\hline KL4 & 1,8 & 2,8 & 3,8 & & 2,7 & & & & \\
\hline & $\begin{array}{l}21,15 \\
22,13\end{array}$ & $\begin{array}{l}22,15 \\
23,13\end{array}$ & $\begin{array}{l}23,15 \\
20,12\end{array}$ & $\begin{array}{l}20,14 \\
21,12\end{array}$ & $\begin{array}{l}21,14 \\
22,12\end{array}$ & $\begin{array}{l}22,14 \\
23,12\end{array}$ & $\begin{array}{l}23,14 \\
21,11\end{array}$ & $\begin{array}{l}20,13 \\
22,11\end{array}$ & 21,13 \\
\hline WA2 & 19,12 & 20,12 & 18,11 & & 20,11 & 18,10 & 10 & 18,9 & 19, \\
\hline$W$ & 11 & 18,11 & 16,10 & 10 & 18,10 & 17,9 & 18,9 & 17,8 & 18,8 \\
\hline & 20,10 & 19,9 & & & & 20,8 & & & \\
\hline & & & & & 21,10 & 22,10 & & 21,9 & 22,9 \\
\hline & & & & & & & & & \\
\hline WAT & & 25,10 & 26,10 & 24,9 & 25,9 & 26,9 & 24,8 & 25,8 & 26,8 \\
\hline
\end{tabular}


TABLE 2. (contd)

Census

Div. $\quad$ MESOILT2 NODES

\begin{tabular}{|c|c|c|c|c|c|c|c|c|c|}
\hline $\begin{array}{l}\text { WA8 } \\
\text { WA9 }\end{array}$ & $\begin{array}{l}23,9 \\
20,8\end{array}$ & $\begin{array}{l}24,9 \\
21,8\end{array}$ & $\begin{array}{l}22,8 \\
22,8\end{array}$ & $\begin{array}{l}23,8 \\
20,7\end{array}$ & $\begin{array}{l}24,8 \\
21,7\end{array}$ & $\begin{array}{l}22,7 \\
22,7\end{array}$ & 23,7 & 24,7 & \\
\hline $\begin{array}{l}\text { A10 } \\
\text { A1 }\end{array}$ & $\begin{array}{r}22,9 \\
2,17 \\
5,16\end{array}$ & $\begin{array}{r}23,9 \\
3,17 \\
6,16\end{array}$ & $\begin{array}{r}22,8 \\
4,17 \\
7,16\end{array}$ & $\begin{array}{r}23,8 \\
5,17 \\
8,16\end{array}$ & $\begin{array}{r}22,7 \\
6,17\end{array}$ & $\begin{array}{r}23,7 \\
7,17\end{array}$ & 2,16 & 3,16 & 4,16 \\
\hline $\begin{array}{l}\text { A2 } \\
\text { A5 }\end{array}$ & $\begin{array}{l}1,19 \\
1,16\end{array}$ & $\begin{array}{l}2,19 \\
2,16\end{array}$ & $\begin{array}{l}1,18 \\
1,15\end{array}$ & $\begin{array}{l}2,18 \\
2,15\end{array}$ & 1,17 & 2,17 & 1,16 & 2,16 & \\
\hline & $\begin{array}{l}2,15 \\
8,14\end{array}$ & 3,1 & 4,15 & & 7,15 & 8,15 & 5,14 & 6,14 & 7,14 \\
\hline YA7 & $\begin{array}{l}1,15 \\
3,13\end{array}$ & $\begin{array}{l}2,15 \\
4,13\end{array}$ & $\begin{array}{l}1,14 \\
5,13\end{array}$ & $\begin{array}{l}2,14 \\
1,12\end{array}$ & $\begin{array}{l}3,14 \\
2,12\end{array}$ & $\begin{array}{l}4,14 \\
3,12\end{array}$ & 5,14 & 1,13 & 2,13 \\
\hline YA9 & $\begin{array}{l}6,1 \\
8,1\end{array}$ & 7,1 & & & & & 7,12 & 8,12 & 7,11 \\
\hline $\begin{array}{l}\text { YA10 } \\
\text { YAI } 1\end{array}$ & $\begin{array}{l}7,11 \\
1,12 \\
6,11 \\
3,9\end{array}$ & $\begin{array}{l}6,10 \\
2,12 \\
1,10 \\
4,9\end{array}$ & $\begin{array}{l}7,10 \\
4,12 \\
2,10 \\
5,9\end{array}$ & $\begin{array}{l}8,10 \\
5,12 \\
3,10\end{array}$ & $\begin{array}{l}5,9 \\
1,11 \\
4,10\end{array}$ & $\begin{array}{l}6,9 \\
2,11 \\
5,10\end{array}$ & $\begin{array}{l}7,9 \\
3,11 \\
6,10\end{array}$ & $\begin{array}{l}8,9 \\
4,11 \\
1,9\end{array}$ & $\begin{array}{l}5,11 \\
2,9\end{array}$ \\
\hline 101 & $\begin{array}{r}10,6 \\
9,4\end{array}$ & $\begin{array}{l}11,6 \\
10,4\end{array}$ & $\begin{array}{r}7,5 \\
11,4\end{array}$ & $\begin{array}{l}8,5 \\
7,3\end{array}$ & $\begin{array}{l}9,5 \\
8,3\end{array}$ & $\begin{array}{r}10,5 \\
9,3\end{array}$ & $\begin{array}{l}11,5 \\
10,3\end{array}$ & 7,4 & 8, \\
\hline 02 & 11, & 12,2 & 13,2 & 10,1 & 11,1 & & 13,1 & & \\
\hline 03 & 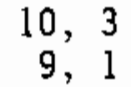 & $\begin{array}{l}11,3 \\
10,1\end{array}$ & & & 9,2 & & 11,2 & 7,1 & 8 \\
\hline IMI & $\begin{array}{l}19,7 \\
21,5\end{array}$ & $\begin{array}{l}20,7 \\
20,4\end{array}$ & 21,7 & 19,6 & 20,6 & 21,6 & 22,6 & 23,6 & 20 \\
\hline M2 & 21,8 & 22,8 & 23,8 & & & & 21,6 & 22,6 & 23, \\
\hline JM4 & $\begin{array}{l}16,7 \\
17,5\end{array}$ & $\begin{array}{l}17,7 \\
18,5\end{array}$ & $\begin{array}{l}18,7 \\
19,5\end{array}$ & $\begin{array}{l}16,6 \\
17,4\end{array}$ & & & $\begin{array}{l}19,6 \\
18,3\end{array}$ & 15,5 & 16, \\
\hline M5 & 21 & & 23,7 & 21,6 & & & 21,5 & 22 & \\
\hline & & & 19,4 & & & & & & \\
\hline $\mathrm{UM} /$ & & $\begin{array}{l}13,4 \\
15,2\end{array}$ & $\begin{array}{l}12,3 \\
16,2\end{array}$ & $\begin{array}{l}13,3 \\
17,2\end{array}$ & $\begin{array}{l}14,3 \\
18,2\end{array}$ & $\begin{array}{l}15,3 \\
14,1\end{array}$ & $\begin{array}{l}16,3 \\
15,\end{array}$ & $\begin{array}{l}12,2 \\
16,1\end{array}$ & $\begin{array}{l}13 \\
17\end{array}$ \\
\hline JM8 & $\begin{array}{l}21,4 \\
21,2\end{array}$ & $\begin{array}{l}22,4 \\
22, \quad 2\end{array}$ & $\begin{array}{l}23,4 \\
19,1\end{array}$ & 19,3 & 20,3 & 21,3 & 22,3 & 19,2 & 20 \\
\hline UM & & & & & & & & & \\
\hline & $\begin{array}{l}23 \\
24\end{array}$ & & & & & & 21,1 & 22,1 & 23 \\
\hline $\begin{array}{l}\text { UM11 } \\
\text { UM12 } \\
\text { UM13 } \\
\text { UM14 }\end{array}$ & $\begin{array}{l}20,8 \\
12,7 \\
23,7 \\
22,5\end{array}$ & $\begin{array}{l}21,8 \\
13,7 \\
24,7 \\
23,5\end{array}$ & $\begin{array}{l}22,8 \\
14,7 \\
25,7 \\
24,5\end{array}$ & $\begin{array}{ll}20, & 7 \\
12, & 6 \\
22, & 6 \\
22, & 4\end{array}$ & $\begin{array}{l}21,7 \\
13,6 \\
23,6 \\
23,4\end{array}$ & $\begin{array}{ll}22, & 7 \\
14, & 6 \\
24, & 6 \\
24, & 4\end{array}$ & $\begin{array}{ll}21, & 6 \\
12, & 5 \\
25, & 6 \\
25, & 4\end{array}$ & $\begin{array}{ll}22, & 6 \\
13, & 5 \\
24, & 5\end{array}$ & 25 \\
\hline
\end{tabular}





\section{MODEL RESULTS}

MESOILT2 was used to compute the monthly time-integrated atmospheric concentrations, monthly deposition rates, and month-end surface contamination at nodes within the model domain for each month from January 1983 , through December 1987. The calculations provide data on typical atmospheric transport and diffusion for the study area that can be used to estimate potential air concentrations, deposition rates, and surface contamination from December 1944 through 1947 when combined with estimates of the actual release magnitudes. In Phase I of the study, the pairing of release data and the atmospheric transport and diffusion calculations will be done in another HEDR Project task. In later phases, the release data will be used as input to the atmospheric mode?.

Dimensions of several of the census divisions are smaller than can be resolved on the MESOILT2 grid. Computations for these divisions are made using nodes surrounding the division. In each of these cases, there was little variation in the computed values at the nodes surrounding the census division. As a result, inability to resolve the census division did not hinder the process of estimating air concentrations and surface contamination in the census division. Similarly, there are two instances where it is not possible to distinguish between two divisions (Ellensburg and Kittitas Division 9, Walla Walla Division 9, and College Place). In each case, a single set of computations is used to characterize the atmospheric transport and deposition for both points. Finally, there are several census divisions that are entirely off the MESOILT2 grid. In each of these cases, the average air concentrations, deposition rates, and surface contamination in the divisions should be lower than in nearby divisions for which computations have been made.

Examination of early MESOILT2 output indicated that monthly timeintegrated concentrations at nodes within a small area have an approximately log-normal distribution. Analysis of the Phase I results indicates that this assumption is not generally appropriate. On the basis of the log-normal assumption, the logarithms (base 10) of the time-integrated concentrations, deposition rates and surface contaminations were used in computing census 
division averages. Nodes in the MESOILT2 domain that did not experience any airborne concentrations or deposition during specific months were not considered in computing the census division values because the logarithm of zero is undefined. To avoid biasing the averages by including logarithms of extremely small values, threshold values were established. Values below the threshold were assumed to be zero and were ignored in the census division calculations. The threshold value assumed for time-integrated concentrations was $1.0 \times 10^{-14}(\mathrm{Ci}-\mathrm{s}) / \mathrm{m}^{3}$. For deposition rates, the threshold values was $1.0 \times 10^{-20}\left(\mathrm{Ci} / \mathrm{m}^{2}\right) / \mathrm{s}$, and for surface contamination the threshold value was $1.0 \times 10^{-16} \mathrm{ci} / \mathrm{m}^{2}$.

The PHASE 11 post-processor computer program computes the variances of the monthly values at the nodes associated with each division as well as the average values. These variances are a measure of the uncertainty of the census division values resulting from spatial variations in the MESOILT2 output each year and from the year-to-year variations in the meteorologica? conditions. A second variance has been added to each computed variance to account for basic uncertainties in atmospheric transport and dispersion modeling. The variance of the differences of the logarithms of predicted and observed concentrations in the data set discussed by Ramsdell (1991, Appendix c) is 0.13 . This variance is associated with predictions of monthly average concentrations of a noble gas. Variances in predicted concentrations and surface contamination for a depositing material are likely to be larger than for nondepositing material. Therefore, variance of 0.26 has been assumed to represent basic modeling uncertainty.

Finally, the post-processing program normalizes all monthiy atmospheric concentrations and surface contamination values to a $1 \mathrm{Ci}$ release. This normalization permits estimation of the time-integrated concentrations, deposition rates and surface contamination for a specific month by multiplying the reported values by the actual monthly release.

Tables 3 through 14 contain the atmospheric transport modeling results for the census divisions for Phase I. Each table lists the census divisions and the average and standard deviation (square roots of the variance) of the time-integrated air concentrations, monthly-average deposition rates, and month-end surface contamination for each division. Logarithms presented in 
the tables are direct listings of files generated by the PHASElI program. These files were transmitted electronically to others for use in the dose calculations.

The results in Tables 3 through 14 are typical values and are not final HEDR estimates. The final estimates will be based on actual release data and concurrent meteorological data. 
TABLE 3. Computational Results for January. All numerical values are logarithms to base 10. Units are as follows: time-integrated air concentration, $\left[(\mathrm{Ci}-\mathrm{s}) / \mathrm{m}^{3}\right] / \mathrm{C}$; deposition rate, $\left[\left(\mathrm{Ci} / \mathrm{m}^{2}\right) / \mathrm{s}\right] / \mathrm{C}$; surface contamination, $\left(\mathrm{Ci} / \mathrm{m}^{2}\right) / \mathrm{Ci}$.

Time-Integrated

Census Air Concentration$$
\text { D }
$$

ADI

$A D 2$

AD3

AD4

AD5

AD6

AD7

AD8

AD9

AD10

BE 1

BE2

BE3

BE4

BE5

BE6

BE7

BE8

BE9

FR1

FR2

FR3

FR4

FR5

GR2

GR3

GR4

GR5

GR6

GR7

GR8

GR9

GR10

GR11

GR12

GR13

GR14

KII

$\mathrm{KI} 2$

KI7

KI8

KI9

KI10

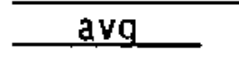

$-10.15197$

$-10.28782$

$-9.93091$

$-10.12580$

$-10.20640$

$-10.66556$

$-10.56948$

$-10.29150$

$-9.69502$

$-9.30515$

$-8.39489$

$-7.79503$

$-8.14036$

$-8.33021$

$-9.52384$

$-10.29237$

$-7.92393$

$-9.70893$

$-8.22518$

$-9.11467$

$-9.49968$

$-8.14882$

$-8.67165$

$-8.15271$

$-10.76531$

$-10.88795$

$-10.42454$

$-11.14198$

$-11.26440$

$-9.85076$

$-10.78870$

$-9.32422$

$-9.33413$

$-10.21687$

$-9.50630$

$-8.67626$

$-11.14128$

$-11.12438$

$-11.40591$

$-12.31210$

$-10.40978$

$-11.79843$

$-12.68366$

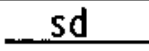

0.57441

1.29403

1.55912

1.42610

I. 11739

1.75045

1.72384

1.17038

0.73491

0.74771

0.61419

0.76935

0.53965

0.54511

1.21981

1.46701

0.54651

1.64170

0.58502

0.84369

0.93892

0.55898

0.76907

0.61651

1.01417

1.61715

1.67226

1.86922

0.95239

0.65556

0.70045

1.30875

1. 38074

0.72708

1.70644

1.88725

2.67191

2.85132

1. 42847

1.97150

1.91807
1.81367

1.78338
Deposition Rate

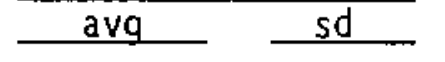

$-18.57985$

$-18.55376$

$-18.12964$

$-18.42792$

$-18.63427$

$-18.91208$

$-18.70481$

$-18.71938$

$-18.12290$

$-17.73302$

$-16.82277$

$-16.22291$

$-16.56824$

$-16.75809$

$-17.89374$

$-18.56883$

$-16.35181$

$-17.93603$

$-16.65306$

$-17.54254$

$-17.92755$

$-16.57669$

$-17.09953$

$-16.58059$

$-19.19319$

$-19.05810$

$-18.61443$

$-19.31120$

$-19.37806$

$-18.27864$

$-18.94316$

$-17.75209$

$-17.76200$

$-18.64475$

$-17.93417$

$-17.10413$

$-19.45979$

$-19.29122$

$-18.60155$

$-19.26469$

$-18.77276$

$-19.76818$

$-20.38654$

\subsection{9}

0.92372

I. 04850

1.16042

1.11742

1.46370

1.22846

1.17041

0.73489

0.74790

0.61418

0.76932

0.53965

0.54505

1.09359

1.22767

0.54645

1.25137

0.58507

0.84367

0.93888

0.55901

0.76912

0.61649

1.01410

1.20625

1.28555

1.55725

1.48570

0.95237

1.43775

0.65554

0.70060

1.30874

1.38071

0.72710

1.59140

1.58060

0.55972

1.78265

1. 32781

1.54718

1.37937
Surface Contamination avg sd

$-12.61925$

$-12.71122$

$-12.32723$

$-12.52606$

$-12.62571$

$-13.08766$

$-13.05411$

$-12.75071$

$-12.12589$

$-11.76698$

$-10.86271$

$-10.27509$

$-10.63684$

$-10.81201$

$-12.14002$

$-12.94341$

$-10.43322$

$-12.31159$

$-10.75054$

$-11.54341$

$-11.85453$

$-10.64049$

$-11.06400$

$-10.61261$

$-13.30512$

$-13.38668$

$-12.86380$

$-13.80392$

$-13.81228$

$-12.21785$

$-13.21154$

$-11.72994$

$-11.75204$

$-12.78592$

$-12.04371$

$-11.19471$

$-13.80083$

$-13.76820$

$-13.62702$

$-14.74576$

$-13.01203$

$-14.41791$

$-15.27615$

0.59709

1.21705 
IABLE 3. (contd)

\begin{tabular}{|c|c|c|c|c|c|c|}
\hline \multirow{2}{*}{$\begin{array}{l}\text { Census } \\
\text { Div. }\end{array}$} & \multicolumn{2}{|c|}{$\begin{array}{l}\text { Time-Integrated } \\
\text { Air Concentration }\end{array}$} & \multicolumn{2}{|c|}{ Deposition Rate } & \multicolumn{2}{|c|}{$\begin{array}{c}\text { Surface } \\
\text { Contamination }\end{array}$} \\
\hline & avg & sd & avg & sd & avg & sd \\
\hline $\mathrm{KL} 1$ & -12.08374 & 1.92741 & -20.09815 & 1.58685 & -14.77357 & 1.92538 \\
\hline $\mathrm{KL} 2$ & -11.97676 & 2.28182 & -19.69351 & 1.56385 & -14.25952 & 1.90392 \\
\hline$K L 3$ & -12.78660 & 1.79898 & -20.91735 & 1.50162 & -15.23445 & 1.63836 \\
\hline KL4 & -13.09519 & 1.75029 & -20.81571 & 1.15021 & -15.86126 & 1.82931 \\
\hline WA 1 & -9.95341 & 0.80329 & -18.38128 & 0.80330 & -12.26655 & 0.73811 \\
\hline WA2 & -9.31538 & 0.82527 & -17.74325 & 0.82528 & -11.72151 & 0.80443 \\
\hline WA3 & -8.70533 & 0.62292 & -17.13321 & 0.62294 & -11.12731 & 0.61695 \\
\hline WA4 & -9.12346 & 0.73408 & -17.55134 & 0.73399 & -11.52544 & 0.71621 \\
\hline WA5 & -9.73731 & 0.76199 & -18.16518 & 0.76202 & -12.13587 & 0.76717 \\
\hline WA6 & -10.10952 & 0.69618 & -18.53740 & 0.69616 & -12.44275 & 0.68036 \\
\hline WA7 & -10.38593 & 0.65784 & -18.81381 & 0.65780 & -12.67225 & 0.62223 \\
\hline WA8 & -9.81930 & 0.75410 & -18.24717 & 0.75409 & -12.14759 & 0.75696 \\
\hline WA9 & -9.37132 & 0.76567 & -17.79919 & 0.76572 & -11.73182 & 0.73178 \\
\hline WAIO & -9.70040 & 0.75540 & -18.12828 & 0.75547 & -12.04228 & 0.76319 \\
\hline YAl & -10.91831 & 1.74677 & -19.11138 & 1.45957 & -13.53538 & 1.80827 \\
\hline YA2 & -13.05000 & 1.82788 & -20.65008 & I. 24270 & -15.73034 & 1.78180 \\
\hline YA5 & -12.86569 & 1.31376 & -20.96326 & 0.99835 & -15.69398 & 1.16112 \\
\hline YA6 & -10.57660 & 1.62520 & -18.79700 & 1.31870 & -13.29039 & 1.67739 \\
\hline YA7 & -12.22334 & 1.34222 & -20.55053 & 1.24229 & -15.03351 & 1.38762 \\
\hline YA9 & -10.25700 & 1.56971 & -18.46576 & 1.15209 & -12.98646 & 1.57648 \\
\hline YA10 & -11.20826 & 1.90860 & -19.23755 & 1.37024 & -13.81086 & 1.70131 \\
\hline YAIl & -12.44404 & 1.75110 & -20.49102 & 1.41659 & -15.12203 & 1.70521 \\
\hline M0l & -10.52137 & 1.54546 & -18.73410 & 1.16662 & -13.20607 & 1.62852 \\
\hline M02 & -10.75296 & 1.54173 & -19.18083 & 1.54176 & -13.47446 & 1.71410 \\
\hline M03 & -11.24679 & 2.16472 & -18.94606 & 1.29706 & -13.87762 & 2.20185 \\
\hline UMI & -9.19309 & 0.70551 & -17.62097 & 0.70541 & -11.57760 & 0.68893 \\
\hline UM2 & -9.52948 & 0.78660 & -17.95735 & 0.78660 & -11.87345 & 0.76426 \\
\hline UM3 & .51279 & 0.74079 & -17.94066 & 0.74082 & -12.15221 & 0.86397 \\
\hline UM4 & -8.88993 & 0.56319 & -17.31780 & 0.56339 & -11.34073 & 0.57950 \\
\hline UM5 & .48158 & 0.78767 & -17.90945 & 0.78764 & -11.83759 & 0.76714 \\
\hline UM6 & -9.11024 & 403 & -17.53812 & 0.57393 & -11.53027 & 0.58567 \\
\hline UM7 & -9.96766 & 0.95805 & -18.39553 & 0.95802 & -12.54857 & 1.07867 \\
\hline UM8 & -9.22254 & 0.60886 & -17.65041 & 0.60887 & -11.61478 & 0.61442 \\
\hline UM9 & -9.83209 & 0.85976 & -18.25997 & 0.85976 & -12.52211 & 0.99244 \\
\hline UM10 & 59050 & 0.70453 & -18.01838 & 0.70453 & -12.01197 & 0.69566 \\
\hline UMl l & .38243 & 0.77261 & -17.81031 & 0.77256 & -11.74584 & 0.74138 \\
\hline UM12 & .49963 & 0.75946 & -17.92751 & 0.75937 & -12.16740 & 0.88053 \\
\hline UM13 & & 0.77972 & -18.16940 & 0.77968 & -12.05338 & 0.77525 \\
\hline UMl 4 & -9 & 0.79428 & -18.04843 & 0.79424 & -11.98300 & 0.78807 \\
\hline
\end{tabular}


IABLE 4. Computational Results for February. All numerical values are logarithms to base 10. Unitş are as follows: time-integrąted air concentration, $\left[(\mathrm{Ci}-\mathrm{s}) / \mathrm{m}_{2}\right] / \mathrm{Ci}$; deposition rate, $\left[\left(\mathrm{Ci} / \mathrm{m}^{2}\right) / \mathrm{s}\right] / \mathrm{Ci}$; surface contamination, $\left(\mathrm{Ci} / \mathrm{m}^{2}\right) / \mathrm{Ci}$.

Time-Integrated

Census Air Concentration

$A D$

$A D 2$

$A D 3$

AD4

AD5

AD6

AD7

AD8

AD9

AD10

BE 1

BE2

BE3

BE4

BE5

BE6

BE7

BE8

BE9

FR1

FR2

FR3

FR4

FR5

GR2

GR3

GR4

GR5

GR6

GR7

GR8

GR9

GR10

GR11

GR12

GR13

GR14

$\mathrm{KI}]$

$\mathrm{KI} 2$

KI7

$\mathrm{KI} 8$

KI9

KIIO \begin{tabular}{c}
\hline avg \\
\hline-10.47975 \\
-10.17679 \\
-9.82326 \\
-9.82295 \\
-9.95648 \\
-10.04030 \\
-10.05999 \\
-9.84142
\end{tabular}

$-9.60532$

$-9.17846$

$-8.58768$

$-7.85519$

$-8.22741$

$-8.38660$

$-9.41518$

$-9.94303$

$-8.01694$

$-9.47827$

$-8.31859$

$-8.95355$

$-9.24205$

$-8.23839$

$-8.51753$

$-8.15990$

$-10.21422$

$-9.93029$

$-9.62815$

$-9.72949$

$-9.86674$

$-9.32408$

$-9.58250$

$-9.26239$

$-9.36731$

$-9.43785$

$-9.20497$

$-8.81709$

$-9.75856$

$-11.41109$

$-12.01625$

$-11.83507$

$-10.94832$

$-11.38401$

$-11.58982$

\begin{tabular}{ccc} 
sd & & avg \\
0.68574 & & -18.86647 \\
0.58807 & & -18.56351 \\
0.57924 & & -18.20998 \\
0.68963 & -18.20968 \\
0.60961 & & -18.34320 \\
0.56682 & -18.42703 \\
0.59641 & -18.44671 \\
0.55985 & -18.22813 \\
0.63227 & -17.99205 \\
0.67132 & -17.56518 \\
0.67460 & -16.97440 \\
0.77246 & -16.24191 \\
0.55023 & -16.61413 \\
0.55803 & -16.77332 \\
1.01850 & -17.80190 \\
1.32849 & -18.32975 \\
0.56483 & -16.40366 \\
1.25240 & -17.78991 \\
0.63070 & -16.70531 \\
0.67429 & -17.34027 \\
0.72075 & -17.62876 \\
0.55185 & -16.62511 \\
0.62878 & -16.90425 \\
0.54290 & -16.54662 \\
0.69327 & -18.60094 \\
0.63312 & -18.31701 \\
0.59472 & -18.01487 \\
0.66043 & -18.11621 \\
0.67920 & -18.25346 \\
0.59470 & -17.71081 \\
0.63230 & -17.96922 \\
0.57512 & -17.64912 \\
0.61511 & -17.75403 \\
0.69824 & -17.82457 \\
0.99035 & -17.59169 \\
1.02923 & -17.20395 \\
0.64168 & -18.14529 \\
1.49442 & -19.73138 \\
1.20531 & -20.39992 \\
1.02078 & -20.21874 \\
1.73997 & -19.15877 \\
0.91769 & -19.76768 \\
1.04512 & -19.97349 \\
& \\
\hline
\end{tabular}

$\frac{\text { Deposition Rate }}{\text { avg }}$

0.68540

0.58816

0.57798

0.68834

0.60977

0.56719

0.59666

0.55980

0.63261

0.67089

0.67459

0.77238

0.55009

0.55783

1.01789

1.32727

0.56458

1.08150

0.63012

0.67459

0.72091

0.55151

0.62932

0.54303

0.69236

0.63252

0.59436

0.65849

0.67784

0.59395

0.63167

0.57446

0.61421

0.69673

0.98956

1.02948

0.64000

1.41385

1.20529

1.02075

1.49185

0.91767

1.04512
Surface Contamination

Cavg__ sd

$-12.86166$

$-12.57822$

0.71994

$-12.22847$

0.63550

$-12.21373$

$-12.36434$

$-12.45253$

$-12.44056$

$-12.18628$

$-11.95793$

$-11.53134$

$-11.07874$

$-10.29728$

$-10.73541$

$-10.87530$

0.61411

0.75790

0.64739

0.58239

0.59793

0.54944

0.61788

0.67698

0.62563

0.76866

0.55729

0.55333

$-11.87952$

0.97326

$-12.42472$

1.22269

$\begin{array}{ll}-10.50002 & 0.56986\end{array}$

$-12.01871$

$-10.80865$

1.28208

0.61498

$-11.29477$

0.66441

$-11.59252$

0.70319

$-10.71823$

0.54648

$-10.88768$

0.61920

$-10.58476$

0.54787

$-12.69135$

0.71746

$-12.41519$

0.68155

$-12.07582$

0.66389

$-12.18994$

0.73202

0.71881

$-12.32641$

0.64882

0.72390

0.62616

0.66495

0.72097

1.00628

1.05917

0.72105

$-12.22143$

1.43835

$-13.94037$

1.31126

$-14.43792$

1.11761

$-13.46699$

1.67359

$-14.00017$

1.00083

$-14.20484$

I. 14908 
TABLE 4. (contd)

\begin{tabular}{|c|c|c|c|c|c|c|}
\hline \multirow{2}{*}{$\begin{array}{l}\text { Census } \\
\text { Div. }\end{array}$} & \multicolumn{2}{|c|}{$\begin{array}{l}\text { Time-Integrated } \\
\text { Air Concentration }\end{array}$} & \multicolumn{2}{|c|}{ Deposition Rate } & \multicolumn{2}{|c|}{$\begin{array}{c}\text { Surface } \\
\text { Contamination }\end{array}$} \\
\hline & $a v g$ & sd & avg & $s d$ & avg & sd \\
\hline KL1 & -11.40012 & 1.73965 & -19.44127 & 1.28691 & -13.86582 & 1.78628 \\
\hline KL2 & -10.75917 & 1.33844 & -19.14538 & 1.34106 & -13.29696 & 1.57344 \\
\hline KL3 & -11.33662 & 0.90739 & -19.72029 & 0.90737 & -13.75639 & 1.07663 \\
\hline KL4 & -11.58687 & 0.55253 & -19.97054 & 0.55250 & -14.01238 & 0.64599 \\
\hline WA1 & -9.58805 & 0.59917 & -17.97476 & 0.59891 & -11.89251 & 0.59734 \\
\hline WA2 & -8.98508 & 0.57364 & -17.37181 & 0.57436 & -11.37569 & 0.56620 \\
\hline WA3 & -8.69163 & 0.52720 & -17.07835 & 0.52718 & -11.12679 & 0.53698 \\
\hline WA4 & -8.98415 & 0.54252 & -17.37087 & 0.54314 & -11.41847 & 0.55209 \\
\hline WA5 & -9.32713 & 0.57627 & -17.71385 & 0.57748 & -11.74819 & 0.57617 \\
\hline WA6 & -9.59388 & 0.56350 & -17.98060 & 0.56414 & -11.97468 & 0.56858 \\
\hline WA7 & -9.81141 & 0.57245 & -18.19813 & 0.57367 & -12.18631 & 0.60231 \\
\hline WA8 & -9.45999 & 0.57473 & -17.84671 & 0.57603 & -11.88658 & 0.59127 \\
\hline WA9 & -9.16716 & 0.53295 & -17.55388 & 0.53328 & -11.58156 & 0.53579 \\
\hline WA10 & -9.36470 & 0.55483 & -17.75142 & 0.55622 & -11.79813 & 0.56019 \\
\hline YAl & -10.75911 & 1.92663 & -18.90967 & 1.60366 & -13.28062 & 1.91144 \\
\hline YA2 & -12.55860 & 1.47862 & -20.72481 & 1.23016 & -15.16803 & 1.51389 \\
\hline YA5 & -14.33762 & 1.31672 & -22.06963 & 0.77579 & -16.94953 & 1.27185 \\
\hline YA6 & -10.52172 & 2.12130 & -18.62173 & 1.71693 & -13.04882 & 2.10532 \\
\hline YA7 & -13.03272 & 1.76158 & -20.85967. & 1.47687 & -15.54096 & 1.80926 \\
\hline YA9 & -9.86352 & 1.19845 & -18.25024 & 1.19768 & -12.37701 & 1.13918 \\
\hline YA10 & -10.42200 & 1.58547 & -18.80926 & 1. 58437 & -12.99450 & 1.56378 \\
\hline YAll & -11.11982 & 1.37472 & -19.43115 & 1. 26953 & -13.60221 & 1.48493 \\
\hline $\mathrm{MOl}$ & -10.78753 & 1.50772 & -19.06641 & 1.37114 & -13.44882 & 1.70608 \\
\hline M02 & -10.23800 & 0.81706 & -18.62473 & 0.81846 & -12.77060 & 0.92020 \\
\hline M03 & -11.11198 & 1.52414 & -19.42618 & 1.44024 & -13.74840 & 1.71305 \\
\hline UMI & -9.14413 & 0.53713 & -17.53085 & 0.53695 & -11.61205 & 0.55005 \\
\hline UM2 & -9.27928 & 0.53909 & -17.66600 & 0.53959 & -11.70409 & 0.54586 \\
\hline UM3 & -9.26177 & 7796 & -17.64849 & 0.67766 & -11.81382 & 0.73622 \\
\hline UM4 & -8.92319 & 363 & -17.30991 & 0.56330 & -11.43688 & 0.58004 \\
\hline UM5 & -9.29147 & 3834 & -17.67819 & 0.53837 & -11.73022 & 0.54620 \\
\hline UM6 & -9.08273 & 944 & -17.46945 & 0.55876 & -11.60255 & 0.57417 \\
\hline UM7 & -9.65365 & 8935 & -18.04037 & 0.68918 & -12.19280 & 0.78089 \\
\hline UM8 & -9.22896 & 5225 & -17.61567 & 0.55176 & -11.73623 & 0.58008 \\
\hline UM9 & -9.57389 & 0.68253 & -17.96061 & 0.68232 & -12.15436 & 0.76010 \\
\hline UM10 & -9.46601 & 0.55690 & -17.85273 & 0.55660 & -11.96386 & 0.58298 \\
\hline UMll & -9.18467 & 0.53428 & -17.57139 & 0.53451 & -11.60811 & 0.54009 \\
\hline UM12 & -9.28740 & 1040 & -17.67412 & 0.68004 & -11.85191 & 0.71638 \\
\hline UM13 & -9.50535 & 126 & -17.89207 & 0.55192 & -11.92333 & 0.57331 \\
\hline UM14 & -9.47973 & 7656 & -17.86645 & 0.57645 & -11.90970 & 0.58011 \\
\hline
\end{tabular}


TABLE 5. Computational Results for March. All numerical values are logarithms to base 10. Units are as follows: time-integrated air concentration, $\left[(\mathrm{Ci}-\mathrm{s}) / \mathrm{m}_{2}^{3}\right] / \mathrm{C}$; deposition rate, $\left[\left(\mathrm{Ci} / \mathrm{m}^{2}\right) / \mathrm{s}\right] / \mathrm{C}$; surface contamination, $\left(\mathrm{Ci} / \mathrm{m}^{2}\right) / \mathrm{Ci}$.

Time-Integrated

\begin{tabular}{lcccc} 
Census & \multicolumn{2}{c}{ Air Concentration } & Depositi \\
\cline { 5 - 5 } Div. & avg & sd & & avg \\
\cline { 1 - 2 } AD1 & -9.89389 & 0.53266 & -18.32177 \\
AD2 & -9.81849 & 0.53589 & -18.24636 \\
AD3 & -9.72150 & 0.55219 & -18.14938 \\
AD4 & -9.52306 & 0.54640 & -17.95093 \\
AD5 & -9.52311 & 0.53205 & -17.95099 \\
AD6 & -9.56186 & 0.53026 & -17.98973 \\
AD7 & -9.58217 & 0.52872 & -18.01004 \\
AD8 & -9.38238 & 0.53095 & -17.81026 \\
AD9 & -9.15643 & 0.53721 & -17.58430 \\
AD10 & -8.95411 & 0.57102 & -17.38199 \\
BE1 & -8.55982 & 0.57531 & -16.98769 \\
BE2 & -7.87104 & 0.71973 & -16.29892 \\
BE3 & -8.26508 & 0.54285 & -16.69295 \\
BE4 & -8.41457 & 0.53989 & -16.84245 \\
BE5 & -9.09817 & 0.67184 & -17.52604 \\
BE6 & -9.41641 & 0.61923 & -17.84428 \\
BE7 & -8.06601 & 0.55328 & -16.49389 \\
BE8 & -9.19929 & 0.69592 & -17.62717 \\
BE9 & -8.41058 & 0.58566 & -16.83846 \\
FR1 & -8.65853 & 0.61945 & -17.08641 \\
FR2 & -8.99822 & 0.59840 & -17.42609 \\
FR3 & -8.26365 & 0.54490 & -16.69152 \\
FR4 & -8.38486 & 0.58784 & -16.81274 \\
FR5 & -8.20931 & 0.55172 & -16.63718 \\
GR2 & -9.94069 & 0.59298 & -18.36857 \\
GR3 & -9.78857 & 0.59087 & -18.21645 \\
GR4 & -9.60691 & 0.58763 & -18.03478 \\
GR5 & -9.53230 & 0.59413 & -17.96017 \\
GR6 & -9.53511 & 0.61487 & -17.96299 \\
GR7 & -9.20677 & 0.56751 & -17.63464 \\
GR8 & -9.47356 & 0.59359 & -17.90143 \\
GR9 & -9.14483 & 0.55610 & -17.57271 \\
GR10 & -9.13802 & 0.55716 & -17.56590 \\
GR11 & -9.15123 & 0.60306 & -17.57911 \\
GR12 & -8.77756 & 0.64112 & -17.20543 \\
GR13 & -8.58126 & 0.78471 & -17.00914 \\
GR14 & -9.53776 & 0.58651 & -17.96564 \\
KI1 & -10.53389 & 1.69564 & -18.81269 \\
KI2 & -12.23033 & 1.90194 & -20.29101 \\
KI7 & -12.02413 & 1.59373 & -20.20664 \\
KI8 & -10.22453 & 1.54975 & -18.58632 \\
KI10 & -11.52488 & 1.70555 & -19.75377 \\
& -1.70460 & 1.83719 & -19.81012
\end{tabular}

Surface Contamination

ava sd

\subsection{9}

0.53590

0.55209

0.54656

0.53199

0.53035

0.52882

0.53110

0.53724

0.57089

0.57526

0.71966

0.54289

0.53996

0.67191

0.61928

0.55327

0.69583

0.58564

0.61947

0.59843

0.54494

0.58775

0.55178

0.59297

0.59078

0.58770

0.59428

0.61493

0.56749

0.59366

0.55605

0.55715

0.60287

0.64116

0.78469

0.58666

1.49927

1.62321

1.38504

1.44220

1.51068

1. 53266
$-12.36057$

$-12.22383$

$-12.06841$

$-11.90734$

$-11.92985$

$-11.98742$

$-12.04107$

$-11.80974$

$-11.58002$

$-11.40661$

$-11.09741$

$-10.36371$

$-10.82812$

$-10.99286$

$-11.59906$

$-11.88154$

$-10.60367$

$-11.66879$

$-10.95731$

$-11.08760$

$-11.40602$

$-10.83087$

$-10.76835$

$-10.66921$

$-12.45181$

$-12.29583$

$-12.05206$

$-12.01091$

$-12.05764$

$-11.66078$

$-11.96342$

$-11.55571$

$-11.52782$

$-11.61389$

$-11.25877$

$-11.13850$

$-12.01973$

$-13.00867$

$-14.76942$

$-14.54559$

$-12.77569$

$-13.98145$

$-14.11918$
0.57331

0.57264

0.57099

0.55120

0.55182

0.54873

0.54055

0.52953

0.53925

0.57323

0.58362

0.73614

0.52995

0.53816

0.66615

0.60388

0.54939

0.63280

0.58091

0.60481

0.60696

0.55466

0.58429

0.55898

0.69427

0.67763

0.66047

0.64066

0.61963

0.58588

0.65848

0.54818

0.55774

0.59553

0.65071

0.84761

0.63297

1.70416

1.92146

1.58917

1. 53731

1.64576

1.74736 
TABLE 5. (contd)

Time-Integrated

Census Air Concentration

-

$\mathrm{KLI}: \frac{\mathrm{avg}}{-11.04037}$

$\begin{array}{ll}K L 2 & -10.90574 \\ \text { KL3 } & -11.94801\end{array}$

$\begin{array}{ll}\text { KL4 } & -11.94801 \\ \text { KA1 } & -9.26153\end{array}$

WAI $\quad-9.26153$

WA2 -8.96202

WA3 -8.73119

WA4 -9.03457

WA5 $\quad-9.19785$

WA6 -9.29430

WA7 $\quad-9.40398$

WA8 $\quad-9.31097$

WA9 -9.22207

WA10 $\quad-9.28674$

YAl -10.20322

YA2 -11.67733

YA5 -11.72100

YA6 $\quad-9.94016$

YA7 -11.31569

YA9 -9.29590

YA10 -9.87200

YA11 -11.01191

M01 $\quad-9.92362$

M02 $\quad-9.96374$

M03 -10.23077

UM1 -9.17726

UM2 -9.28750

UM3 $\quad-9.25649$

UM4 $\quad-8.90566$

UM5 $\quad-9.31560$

UM6 $\quad-9.07188$

UM7 $\quad-9.58420$

UM8 $\quad-9.27090$

$\begin{array}{ll}\text { UM9 } & -9.46500\end{array}$

UMIO $\quad-9.59426$

UM1 $1 \quad-9.23819$

UM12 $\quad-9.27522$

UM13 $\quad-9.42798$

UM14 $\quad-9.52486$ sd

1.15195

0.97237

1.07760

1.21808

0.54950

0.54200

0.55526

0.56187

0.53040

0.52286

0.52168

0.54485

0.55445

0.54415

1.31426

1.70818

1.29632

1.29627

1.31637

0.74849

0.68451

1.33328

0.68259

0.76913

0.80091

0.54059

0.54859

0.56693

0.54541

0.53796

0.54937

0.61828

0.54969

0.56308

0.57575

0.54693

0.56754

0.57143

0.54579
Deposition Rate avg

$-19.46824$

$-19.33362$

$-20.37589$

$-20.48698$

$-17.68940$

$-17.38989$

$-17.15907$

$-17.46245$

$-17.62573$

$-17.72218$

$-17.83185$

$-17.73885$

$-17.64994$

$-17.71462$

$-18.63109$

$-19.74463$

$-20.14887$

$-18.36803$

$-19.69189$

$-17.72378$

$-18.29987$

$-19.40213$

$-18.35150$

$-18.39162$

$-18.65864$

$-17.60513$

$-17.71538$

$-17.68436$

$-17.33354$

$-17.74348$

$-17.49976$

$-18.01208$

$-17.69878$

$-17.89287$

$-18.02213$

$-17.66607$

$-17.70310$

$-17.85585$

$-17.95274$

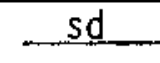

1.15196

0.97235

1.07751

1.21812

0.54959

0.54202

0.55523

0.56181

0.53041

0.52282

0.52173

0.54469

0.55443

0.54407

1.31432

1.32114

1.29637

1.29632

1.25329

0.74840

0.68453

1.28389

0.68268

0.76908

0.80094

0.54055

0.54857

0.56687

0.54539

0.53795

0.54926

0.61834

0.54957

0.56306

0.57574

0.54684

0.56751

0.57153

0.54575
Surface Contamination avg $\mathrm{sd}$

$-13.45869$

1.26073

$-13.36844$

$-14.43783$

$-14.47895$

$-11.66221$

$-11.38330$

$-11.26848$

$-11.52015$

$-11.61331$

$-11.67140$

$-11.76071$

$-11.72695$

$-11.69522$

$-11.71303$

$-12.69657$

$-14.14859$

$-14.26190$

$-12.37587$

$-13.76317$

$-11.80480$

$-12.28989$

$-13.38869$

$-12.33966$

$-12.43898$

$-12.72129$

$-11.76876$

$-11.76034$

$-11.75982$

$-11.51596$

$-11.83013$

$-11.68485$

$-12.06606$

$-11.88185$

$-11.94547$

$-12.15914$

$-11.72816$

$-11.77320$

$-11.87246$

$-12.03889$
1.06800

1.20027

1. 35787

0.55162

0.56037

0.58338

0.59735

0.53181

0.51717

0.52027

0.54103

0.58692

0.54258

1.26816

1.74757

1.39491

1.27007

1.38402

0.76265

0.69171

1.40049

0.64455

0.74730

0.85088

0.55701

0.56563

0.54772

0.54840

0.55958

0.54638

0.58194

0.56032

0.53827

0.59776

0.58118

0.54723

0.57026

0.57036 
IABLE 6. Computational Results for Apri1. All numerical values are logarithms to base 10. Units are as follows: time-integrated air concentration, $\left[(\mathrm{Ci}-\mathrm{s}) / \mathrm{m}_{2}^{3}\right] / \mathrm{C} i$; deposition rate, $\left[\left(\mathrm{Ci} / \mathrm{m}^{2}\right) / \mathrm{s}\right] / \mathrm{C} i$; surface contamination, $\left(\mathrm{Ci} / \mathrm{m}^{2}\right) / \mathrm{Ci}$.

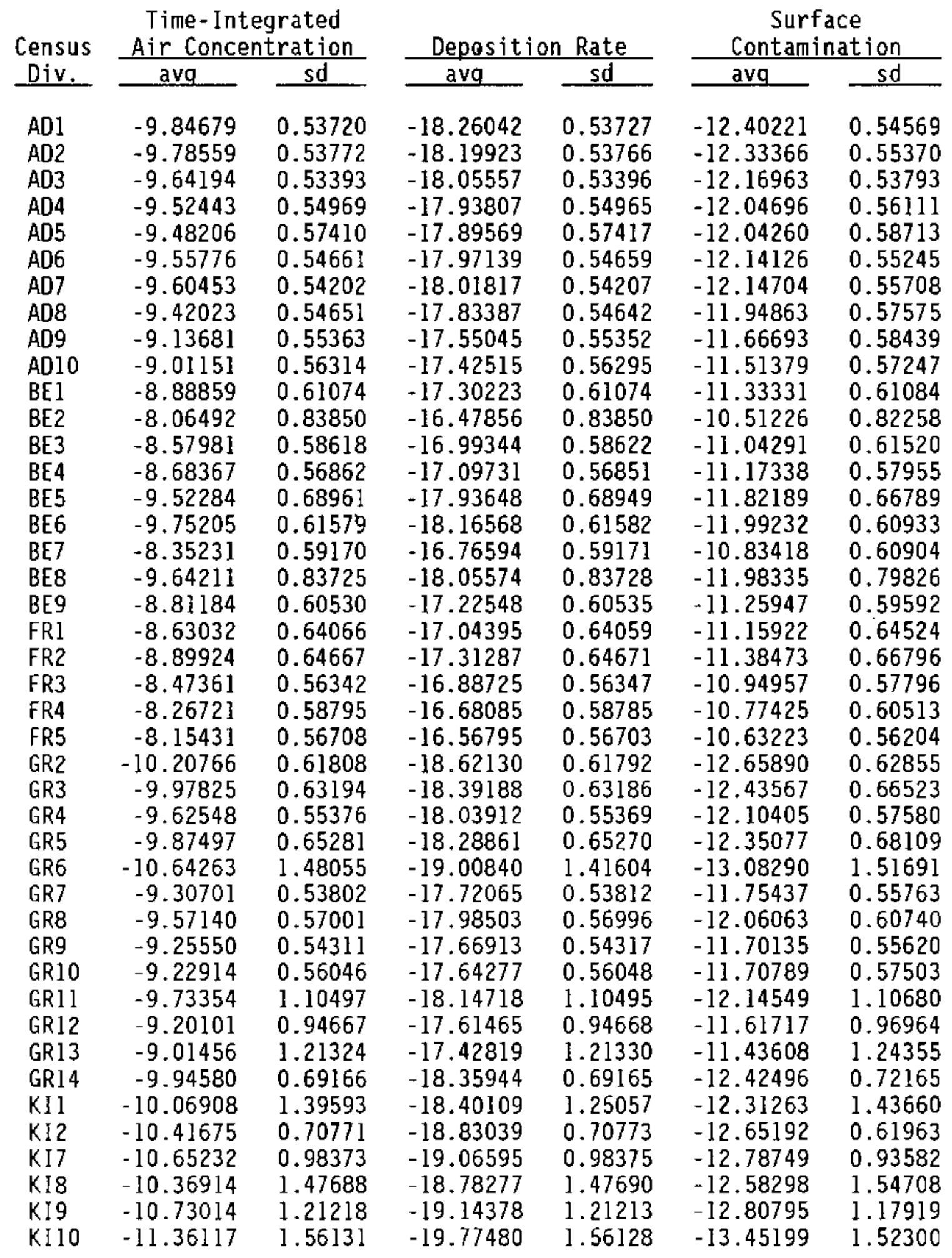


TABLE 6. (contd)

\begin{tabular}{|c|c|c|c|c|c|c|}
\hline \multirow{2}{*}{$\begin{array}{l}\text { Census } \\
\text { Div. }\end{array}$} & \multicolumn{2}{|c|}{$\begin{array}{l}\text { Time-Integrated } \\
\text { Air Concentration }\end{array}$} & \multicolumn{2}{|c|}{ Deposition Rate } & \multicolumn{2}{|c|}{$\begin{array}{c}\text { Surface } \\
\text { Contamination }\end{array}$} \\
\hline & avg & sd & avg & sd & avg & sd \\
\hline KLl & -11.12300 & 1.22403 & -19.48976 & 1.15907 & -13.38507 & 1.23366 \\
\hline $\mathrm{KL} 2$ & -10.97777 & 1.29985 & -19.28738 & 1.13270 & -13.29439 & 1.33457 \\
\hline KL3 & -11.90433 & 1.19327 & -20.31797 & 1.19325 & -14.25251 & 1.04390 \\
\hline KL4 & -12.08423 & 0.91712 & -20.49787 & 0.91704 & -14.37008 & 0.84523 \\
\hline WA1 & -9.11327 & 0.54092 & -17.52691 & 0.54075 & -11.56299 & 0.55508 \\
\hline WA2 & -8.73820 & 0.53881 & -17.15184 & 0.53880 & -11.20114 & 0.54117 \\
\hline WA3 & -8.64316 & 0.55473 & -17.05680 & 0.55460 & -11.11022 & 0.55272 \\
\hline WA4 & -8.85222 & 0.53441 & -17.26586 & 0.53436 & -11.31861 & 0.52975 \\
\hline WA5 & -8.96945 & 0.53847 & -17.38309 & 0.53840 & -11.42011 & 0.53917 \\
\hline WA6 & -9.09694 & 0.54888 & -17.51058 & 0.54890 & -11.52677 & 0.55693 \\
\hline WA7 & -9.17173 & 0.54661 & -17.58536 & 0.54661 & -11.61717 & 0.55774 \\
\hline WA8 & -9.03066 & 0.53944 & -17.44430 & 0.53948 & -11.49044 & 0.53866 \\
\hline WA9 & -8.98650 & 0.53111 & -17.40014 & 0.53110 & -11.45868 & 0.52845 \\
\hline WA10 & -9.01848 & 0.53904 & -17.43211 & 0.53908 & -11.48032 & 0.53656 \\
\hline YA1 & -10.74063 & 1.71145 & -19.06989 & 1.61650 & -12.99564 & 1.73970 \\
\hline YA2 & -12.06096 & 1.74438 & -20.32989 & 1.62600 & -14.22067 & 1.76905 \\
\hline YA5 & -12.19838 & 1.54374 & -20.61202 & 1.54374 & -14.43180 & 1.72414 \\
\hline YA6 & -10.51821 & 1.68209 & -18.80923 & 1.49883 & -12.82806 & 1.71625 \\
\hline YA7 & -11.37687 & 1.38091 & -19.61766 & 1.16049 & -13.56912 & 1.45832 \\
\hline YA9 & -9.95111 & 1.01570 & -18.36475 & 1.01573 & -12.23833 & 1.12497 \\
\hline YAlO & -10.37789 & 1.03706 & -18.79152 & 1.03707 & -12.59814 & 1.11702 \\
\hline YAll & -11.55229 & 1.33310 & -19.92851 & 1.29540 & -13.83329 & 1.51707 \\
\hline M01 & -10.31683 & 34952 & -18.73047 & 0.84964 & -12.62023 & 0.86747 \\
\hline M02 & -10.18912 & 0.62348 & -18.60276 & 0.62338 & -12.54046 & 0.64566 \\
\hline M03 & -10.39682 & 0.72779 & -18.81045 & 0.72773 & -12.72931 & 0.75361 \\
\hline UMI & -9.14614 & 0.54633 & -17.55978 & 0.54631 & -11.58655 & 0.54106 \\
\hline UM2 & -9.05232 & 3558 & -17.46595 & 0.53559 & -11.52024 & 0.53449 \\
\hline UM3 & -9.57509 & 9168 & -17.98872 & 0.59171 & -12.02711 & 0.63514 \\
\hline UM4 & -9.19940 & 543 & -17.61304 & 0.55542 & -11.66741 & 0.55581 \\
\hline UM5 & -9.14446 & 160 & -17.55810 & 0.54162 & -11.60066 & 0.54121 \\
\hline UM6 & -9.35568 & 485 & -17.76931 & 0.54483 & -11.82863 & 0.55198 \\
\hline UM7 & -9.79177 & 124 & -18.20541 & 0.60115 & -12.22664 & 0.63572 \\
\hline UM8 & -9.39313 & 744 & -17.80677 & 0.53744 & -11.84707 & 0.54721 \\
\hline UM9 & -9.74623 & 090 & -18.15987 & 0.59086 & -12.19278 & 0.64233 \\
\hline UM10 & -9.60903 & 5646 & -18.02266 & 0.55646 & -12.06878 & 0.56828 \\
\hline UMl l & -9.02081 & 3771 & -17.43444 & 0.53775 & -11.48855 & 0.53835 \\
\hline UMI 2 & -9.61993 & 943 & -18.03357 & 0.58936 & -12.04933 & 0.62378 \\
\hline UM13 & -9.13853 & 031 & -17.55216 & 0.54030 & -11.60129 & 0.53515 \\
\hline UM1 4 & -9.32563 & 048 & -17.73927 & 0.54047 & -11.76867 & 0.54031 \\
\hline
\end{tabular}


TABLE 7. Computational Results for May. A11 numerical values are logarithms to base 10. Units are as follows: time-integrated air concentration, $\left[(\mathrm{Ci}-\mathrm{s}) / \mathrm{m}_{2}\right] / \mathrm{C}$; deposition rate, $\left[\left(\mathrm{Ci} / \mathrm{m}^{2}\right) / \mathrm{s}\right] / \mathrm{C} ;$; surface contamination, $\left(\mathrm{Ci} / \mathrm{m}^{2}\right) / \mathrm{Ci}$.

Time-Integrated

Census

Div.

$A D 1$

$A 02$

$A D 3$

AD4

AD5

AD6

AD7

AD8

$A D 9$

AD 10

BE 1

BE2

BE3

BE4

BE5

BE6

BE7

BE8

BE9

FR1

FR2

FR3

FR4

FR5

GR2

GR3

GR4

GR5

GR6

GR7

GR8

GR9

GR10

GR11

GR12

GR13

GR14

KI1

$\mathrm{KI} 2$

KI7

KI8

KI9

KIIO Air Concentration avg

$$
-9.70957
$$

$-9.61966$

$-9.53195$

$-9.36961$

$-9.40110$

$-9.51663$

$-9.53568$

$-9.37193$

$-9.12689$

$-9.00595$

$-8.84820$

$-8.09214$

$-8.63201$

$-8.78090$

$-9.30005$

$-9.46308$

$-8.39581$

$-9.37355$

$-8.69281$

$-8.62397$

$-8.84975$

$-8.53362$

$-8.22440$

$-8.21003$

$-10.13939$

$-9.81786$

$-9.49628$

$-9.53225$

$-9.62281$

$-9.21104$

$-9.45544$

$-9.16635$

$-9.14720$

$-9.21535$

$-8.95686$

$-8.82494$

$-9.56402$

$-10.62749$

$-11.57445$

$-11.59039$

$-10.47192$

$-11.66747$

$-11.97972$

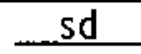

0.52876

0.52964

0.52552

0.53075

0.53124

0.51836

0.51918

0.52127

0.53046

0.54349

0.58208

0.79459

0.54836

0.54444

0.61280

0.60852

0.56419

0.65381

0.55841

0.60552

0.59604

0.53813

0.55809

0.54570

0.59408

0.61309

0.55642

0.55840

0.55823

0.52807

0.55589

0.53077

0.53963

0.55846

0.60720

0.75474

0.54238

1.47981

1.99739

1.94493

1.35062

1.80674

1.79151
Deposition Rate

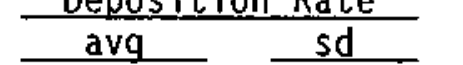

.13744

$-18.04754$

$-17.95983$

$-17.79749$

$-17.82898$

$-17.94451$

$-17.96355$

$-17.79981$

$-17.55477$

$-17.43383$

$-17.27608$

$-16.52001$

$-17.05989$

$-17.20878$

$-17.72791$

$-17.89095$

$-16.82369$

$-17.80143$

$-17.12069$

$-17.05185$

$-17.27763$

$-16.96149$

$-16.65228$

$-16.63791$

$-18.56727$

$-18.24573$

$-17.92416$

$-17.96013$

$-18.05068$

$-17.63892$

$-17.88332$

$-17.59423$

$-17.57508$

$-17.64322$

$-17.38474$

$-17.25282$

$-17.99190$

$-19.05537$

$-20.00233$

$-20.01827$

$-18.89980$

$-20.01320$

$-20.30586$

0.52871

0.52943

0.52558

0.53064

0.53113

0.51830

0.51915

0.52127

0.53044

0.54348

0.58201

0.79461

0.54834

0.54443

0.61304

0.60846

0.56428

0.65373

0.55843

0.60540

0.59615

0.53812

0.55811

0.54575

0.59402

0.61301

0.55644

0.55841

0.55822

0.52810

0.55589

0.53090

0.53965

0.55849

0.60731

0.75470

0.54233

1.47980

1.99736

1.94489

1.35064

1.75876

1.74265
Surface Contamination

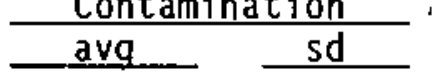

$-12.25809$

$-12.17127$

$-12.05107$

$-11.91208$

$-11.93019$

$-12.05115$

$-12.04152$

$-11.85047$

$-11.60700$

$-11.51206$

$-11.18855$

$-10.53019$

$-11.12828$

$-11.25688$

$-11.70529$

$-11.88092$

$-10.85149$

$-11.77300$

$-11.06891$

$-11.14183$

$-11.33360$

$-11.00971$

$-10.74463$

$-10.67472$

$-12.60797$

$-12.25979$

$-11.96325$

$-12.00706$

$-12.03806$

$-11.68887$

$-11.93208$

$-11.64847$

$-11.65259$

$-11.65731$

$-11.40359$

$-11.27801$

$-12.04314$

$-13.14446$

$-14.18542$

$-14.20211$

$-12.92897$

$-14.19762$

$-14.43767$
0.52108

0.53275

0.54152

0.52689

0.53257

0.52176

0.53059

0.54164

0.53255

0.53319

0.61536

0.77918 
TABLE 7. (contd)

Time-Integrated

Census Air Concentration

Div.

KL1 -11.17873

KL2 -11.01656

KL3 -11.63306

KL4 -11.76572

WA1 $\quad-9.04410$

WA2 -8.78651

WA3 -8.70035

WA4 -8.88767

WA5 -8.97332

WA6 -9.05232

WA7 $\quad-9.14126$

WA8 -9.06618

WA9 $\quad-9.00462$

WA10 $\quad-9.04461$

YAl -10.21479

YA2 -11.68214

YA5 -11.21433

YA6 $\quad-9.81878$

YA7 -10.79798

YA9 $\quad-9.48755$

YA10 -10.01444

YAll -11.00362

M01 $\quad-9.95171$

M02 $\quad-9.81888$

M03 -10.07997

UM1 $\quad-9.15679$

UM2 $\quad-9.07429$

UM3 $\quad-9.33809$

UM4 $\quad-9.22802$

UM5 -9.16595

UM6 $\quad-9.41803$

UM7 $\quad-9.60766$

UM8 $\quad-9.40515$

UM9 $\quad-9.43064$

UM10 -9.52306

UM11 $\quad-9.03831$

UM12 $\quad-9.33473$

UM13 -9.19995

UM14 -9.35648

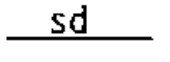

1.43282

1.30017

1.29231

1.57230

0.53520

0.53673

0.54202

0.53016

0.52981

0.52100

0.51757

0.51713

0.51960

0.51843

1.02713

1.46495

1.01470

0.91535

1.12838

0.68470

0.76721

1.26487

0.71870

0.57532

0.62461

0.52583

0.52258

0.55274

0.53536

0.52760

0.54601

0.55957

0.53259

0.54160

0.53956

0.52222

0.56372

0.52743

0.53077

$-\frac{\text { Deposition Rate }}{\text { avg }}$ sd

$-19.56198$

$-19.44444$

$-20.06093$

$-20.02484$

$-17.47198$

$-17.21439$

$-17.12823$

$-17.31555$

$-17.40120$

$-17.48020$

$-17.56914$

$-17.49405$

$-17.43250$

$-17.47248$

$-18.64267$

$-20.11001$

$-19.64221$

$-18.24665$

$-19.22586$

$-17.91542$

$-18.44232$

$-19.43149$

$-18.37958$

$-18.24676$

$-18.50785$

$-17.58467$

$-17.50216$

$-17.76597$

$-17.65589$

$-17.59382$

$-17.84590$

$-18.03554$

$-17.83302$

$-17.85851$

$-17.95094$

$-17.46619$

$-17.76261$

$-17.62782$

$-17.78436$
$1.37871 \cdot-13.49369$

$1.30007-13.34380$

$1.29230-13.91215$

$1.41201-14.04347$

$0.53542-11.53300$

$0.53664-11.25075$

$0.54188-11.16374$

$0.53015-11.34491$

$0.52977-11.43119$

$0.52097-11.50290$

$0.51751-11.60781$

$0.51706-11.50853$

$0.51960-11.45716$

$0.51833-11.48675$

$\begin{array}{ll}1.02709 & -12.65878\end{array}$

$1.46500-14.11968$

$1.01469-13.65107$

$\begin{array}{ll}0.91532 & -12.27897\end{array}$

$1.12838-13.24074$

$0.68469-11.92004$

$\begin{array}{ll}0.76711 & -12.40220\end{array}$

$1.26485-13.38285$

$0.71872-12.30190$

$0.57539-12.14497$

$\begin{array}{lll}0.62463 & -12.38350\end{array}$

$\begin{array}{lll}0.52587 & -11.64351\end{array}$

0.52255

0.55272

0.53550

0.52749

0.54603

0.55939

0.53259

0.54157

0.53948

0.52220

0.56363

0.52741

0.53067
$-11.52331$

$-11.72321$

$-11.73913$

$-11.62814$

$-11.92877$

$-12.03875$

$-11.91342$

$-11.81717$

$-12.01416$

$-11.49297$

$-11.70370$

$-11.63494$

$-11.81919$
1.42751

1.36470

1.21926

1.56124

0.53835

0.56286

0.55653

0.54730

0.55483

0.53008

0.52122

0.52116

0.52485

0.52506

1.08475

1.43407

1.05473

0.94618

1.19790

0.70114

0.81732

1.34233

0.74834

0.56313

0.66315

0.53594

0.52638

0.55108

0.52996

0.53469

0.53029

0.55076

0.53243

0.54061

0.55231

0.52949

0.56026

0.52944

0.54688 
TABLE 8. Computational Results for June. A11 numerical values are logarithms to base 10. Units are as follows: time-integrated air concentration, $\left[(\mathrm{Ci}-\mathrm{s}) / \mathrm{m}_{2}^{3}\right] / \mathrm{Ci}$; deposition rate, $\left[\left(\mathrm{Ci} / \mathrm{m}^{2}\right) / 5\right] / \mathrm{C}$; surface contamination, $\left(\mathrm{Ci} / \mathrm{m}^{2}\right) / \mathrm{Ci}$.

Time-Integrated

Census Air Concentration

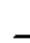

$\mathrm{ADl}$

$\mathrm{AD} 2$

$\mathrm{AD3}$

AD4

$\mathrm{AD5}$

AD6

AD7

AD8

AD9

AD10

$8 \mathrm{E} 1$

BE2

BE3

BE4

BE5

BE6

BE7

BE8

BE9

FR]

FR2

FR3

FR4

FR5

GR2

GR3

GR4

GR5

GR6

GR7

GR8

GR9

GR10

GR11

GR12

GR13

GR14

KII

$\mathrm{KI} 2$

KI7

KI8

$\mathrm{KI} 9$

KI 10

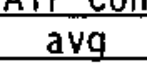

$$
-9.73460
$$

$-9.73377$

$-9.71607$

$-9.50606$

$-9.44702$

$-9.50947$

$-9.50747$

$-9.37271$

$-9.17186$

$-9.03855$

$-9.06887$

$-8.08768$

$-8.61946$

$-8.75069$

$-9.45354$

$-9.71336$

$-8.40610$

$-9.59076$

$-8.81708$

$-8.62731$

$-8.89465$

$-8.53442$

$-8.28293$

$-8.23646$

$-10.33588$

$-10.02369$

$-9.71786$

$-9.67679$

$-9.74192$

$-9.45291$

$-9.63751$

$-9.36027$

$-9.27647$

$-9.28362$

$-8.92886$

$-8.61041$

$-9.71589$

$-10.26106$

$-11.82566$

$-11.61248$

$-9.69331$

$-10.70544$

$-10.24858$

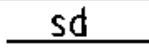

0.58274

0.60170

0.61401

0.59544

0.58685

0.56383

0.54330

0.55130

0.57915

0.57755

0.65950

0.77670

0.55574

0.55036

0.71853

0.68616

0.56168

0.77760

0.61008

0.62564

0.53996

0.57724

0.54479

0.55641

0.55971

0.58881

0.56538

0.57185

0.58170

0.57286

0.56309

0.56064

0.55917

0.57410

0.60818

0.55086

1.36136

2. 12643

2. 16094

1.11702

1.65945

1. 28086
0.60964
Deposition Rate

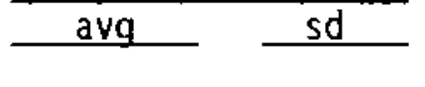

$-18.14824$

$-18.14741$

$-18.12971$

$-17.91970$

$-17.86065$

$-17.92311$

$-17.92111$

$-17.78635$

$-17.58549$

$-17.45219$

$-17.48250$

$-16.50131$

$-17.03309$

$-17.16432$

$-17.86718$

$-18.12700$

$-16.81973$

$-18.00440$

$-17.23071$

$-17.04094$

$-17.30828$

$-16.94805$

$-16.69656$

$-16.65009$

$-18.74951$

$-18.43732$

$-18.13150$

$-18.09043$

$-18.15556$

$-17.86654$

$-18.05114$

$-17.77391$

$-17.69011$

$-17.69725$

$-17.34249$

$-17.02404$

$-18.12953$

$-18.67470$

$-19.85352$

$-19.63054$

$-18.10694$

$-19.00954$

$-18.66222$
0.58269

0.60153

0.61398

0.59544

0.58684

0.56387

0.54325

0.55131

0.57912

0.57764

0.65956

0.77664

0.55578

0.55037

0.71838

0.68609

0.56165

0.77743

0.61007

0.62572

0.60969

0.53993

0.57722

0.54480

0.55652

0.55983

0.58871

0.56533

0.57172

0.58172

0.57295

0.56306

0.56073

0.55906

0.57422

0.60814

0.55084

1. 36140

1.85646

1.88450

1.11694

1.51190

1. 28087
Surface Contamination

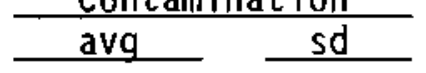

$-12.32297$

0.63407

$-12.28130$

$-12.26428$

$-12.00932$

$-11.96030$

$-12.06505$

$-12.07805$

$-11.91441$

$-11.69495$

$-11.51131$

$-11.44558$

$-10.53961$

$-11.03383$

$-11.17176$

$-11.78683$

$-12.01048$

$-10.84229$

$-11.95191$

$-11.24585$

$-11.13925$

$-11.37012$

$-10.96340$

$-10.76505$

$-10.67883$

$-12.95775$

$-12.63623$

$-12.30036$

$-12.27071$

$-12.34570$

$-11.99973$

$-12.22799$

$-11.86888$

$-11.76130$

$-11.82856$

$-11.39702$

$-11.04507$

$-12.31151$

$-12.76983$

$-14.41498$

$-14.15986$

$-12.09997$

$-13.16141$

$-12.64245$

0.66665

0.71473

0.64112

0.60497

0.58180

0.56866

0.56487

0.58936

0.59541 
IABLE 8. (contd)

\begin{tabular}{|c|c|c|c|c|c|c|}
\hline \multirow{2}{*}{$\begin{array}{l}\text { Census } \\
\text { Div. }\end{array}$} & \multicolumn{2}{|c|}{$\begin{array}{l}\text { Time-Integrated } \\
\text { Air Concentration }\end{array}$} & \multicolumn{2}{|c|}{ Deposition Rate } & \multicolumn{2}{|c|}{$\begin{array}{c}\text { Surface } \\
\text { Contamination }\end{array}$} \\
\hline & avg & $\mathrm{sd}$ & avg & sd & $a v q$ & sd \\
\hline $\mathrm{KLl}$ & -11.17721 & 1. 30055 & -19.54541 & 1.24469 & -13.45755 & 1.31470 \\
\hline KL2 & -11.24272 & 1.44884 & -19.65636 & 1.44886 & -13.56911 & 1.44170 \\
\hline$K L 3$ & -11.53909 & 0.95223 & -19.95273 & 0.95219 & -13.81070 & 1.04970 \\
\hline KL4 & -11.88782 & 1.26946 & -20.14013 & 1.09469 & -14.11938 & 1.29419 \\
\hline WAl & -9.08438 & 0.54885 & -17.49801 & 0.54887 & -11.56317 & 0.54268 \\
\hline WA2 & -8.84001 & 0.53815 & -17.25364 & 0.53820 & -11.29116 & 0.54337 \\
\hline WA3 & -8.73257 & 0.53230 & -17.14621 & 0.53225 & -11.18160 & 0.54283 \\
\hline WA4 & -8.93427 & 0.52613 & -17.34791 & 0.52607 & -11.36522 & 0.53619 \\
\hline WA5 & -9.03003 & 0.53480 & -17.44366 & 0.53476 & -11.47121 & 0.53812 \\
\hline WA6 & -9.16264 & 0.52651 & -17.57628 & 0.52654 & -11.62604 & 0.53058 \\
\hline WA7 & -9.27687 & 0.52200 & -17.69050 & 0.52194 & -11.76096 & 0.52562 \\
\hline WA8 & -9.15506 & 0.52081 & -17.56870 & 0.52062 & -11.62042 & 0.53117 \\
\hline WA9 & -9.08449 & 0.52027 & -17.49812 & 0.52018 & -11.52392 & 0.53300 \\
\hline WA10 & -9.13203 & 0.52080 & -17.54567 & 0.52074 & -11.58712 & 0.53207 \\
\hline YAl & -9.76864 & 1.10624 & -18.18228 & 1.10624 & -12.14731 & 1.10093 \\
\hline YA2 & -10.04597 & 0.75720 & -18.45961 & 0.75724 & -12.38621 & 0.75365 \\
\hline YA5 & -10.22354 & 0.74279 & -18.63718 & 0.74272 & -12.55569 & 0.75047 \\
\hline YA6 & -9.69280 & 1.15379 & -18.10644 & 1.15380 & -12.09609 & 1.18508 \\
\hline YA7 & -10.53224 & 1.17997 & -18.94588 & 1.18003 & -12.89609 & 1.15266 \\
\hline YA9 & -9.58709 & 0.76167 & -18.00073 & 0.76168 & -11.91357 & 0.76069 \\
\hline YA10 & -10.15672 & 0.87503 & -18.57036 & 0.87505 & -12.43217 & 0.87872 \\
\hline YAll & -11.12614 & 1.27195 & -19.50321 & 1.22524 & -13.38480 & 1.27331 \\
\hline M01 & -10.22537 & 0.92764 & -18.63901 & 0.92756 & -12.57550 & 0.93464 \\
\hline M02 & -10.07636 & 0.68754 & -18.49000 & 0.68756 & -12.39466 & 0.70487 \\
\hline M03 & -10.35840 & 0.88297 & -18.77203 & 0.88300 & -12.69210 & 0.88532 \\
\hline UMI & -9.16698 & 0.52586 & -17.58061 & 0.52584 & -11.63637 & 0.53539 \\
\hline UM2 & -9.15394 & 0.52256 & -17.56758 & 0.52241 & -11.61282 & 0.53559 \\
\hline UM3 & -9.57183 & 0.62277 & -17.98546 & 0.62279 & -11.93390 & 0.60895 \\
\hline UM4 & -9.19990 & 0.54902 & -17.61354 & 0.54893 & -11.64398 & 0.54436 \\
\hline UM5 & -9.21589 & 0.52207 & -17.62953 & 0.52203 & -11.68670 & 0.53499 \\
\hline UM6 & -9.37716 & 0.55495 & -17.79080 & 0.55493 & -11.80999 & 0.54090 \\
\hline UM7 & -9.79861 & 1553 & -18.21225 & 0.615 & -12.14212 & 0.61312 \\
\hline UM8 & -9.32734 & 3755 & -17.74097 & 3764 & -11.81140 & 0.54004 \\
\hline UM9 & -9.72343 & 0.62969 & -18.13707 & 0.629 & -12.06008 & 0.62745 \\
\hline UM10 & -9.49086 & 0.55360 & -17.90449 & 0.553 & -11.97566 & 0.54702 \\
\hline UM11 & -9.12336 & 2373 & -17.53700 & 0.523 & -11.57038 & 0.53691 \\
\hline UM12 & -9.60750 & 0.64111 & -18.02114 & 0.64102 & -11.96876 & 0.62042 \\
\hline UMI3 & 477 & 2227 & -17.64841 & 0.52214 & -11.72693 & 0.53813 \\
\hline UMI 4 & -9.3 & 0.52296 & -17.76138 & 0.52299 & -11.84061 & 0.53520 \\
\hline
\end{tabular}


IABLE 9. Computational Results for July. All numerical values are logarithms to base 10 . Units are as follows: time-integrated air concentration, $\left[(\mathrm{C} i-s) / m_{2}^{3}\right] / \mathrm{C}$; deposition rate, $\left[\left(\mathrm{Ci} / \mathrm{m}^{2}\right) / s\right] / \mathrm{Ci}$; surface contamination, $\left(\mathrm{Ci} / \mathrm{m}^{2}\right) / \mathrm{Ci}$.

Time-Integrated

Census Air Concentration

Div.

$\mathrm{AD}$

AD2

$\mathrm{AD} 3$

AD4

$\mathrm{AD5}$

AD6

AD7

AD8

AD9

AD 10

BE 1

BE2

BE3

BE4

BE5

BE6

BE7

BE8

BE9

FRI

FR2

FR3

FR5

GR2

GR3

GR4

GR5

GR6

GR7

GR8

GR9

GR10

GR1 1

GRI2

GR13

GRI 4

KII

$\mathrm{KI} 2$

$\mathrm{KI} 7$

KI8

KI9

KIIO

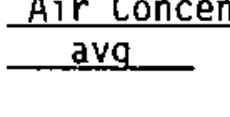

$-10.10926$

$-9.95559$

$-9.85804$

$-9.60876$

$-9.57551$

$-9.68395$

$-9.71459$

$-9.48129$

$-9.28450$

$-9.11457$

$-8.67434$

$-8.04234$

$-8.50317$

$-8.62504$

$-8.99604$

$-9.14800$

$-8.29908$

$-9.14167$

$-8.63461$

$-8.80014$

$-8.91306$

$-8.43066$

$-8.35450$

$-8.13472$

$-10.49134$

$-10.21144$

$-9.84774$

$-9.93492$

$-10.09925$

$-9.51213$

$-9.87232$

$-9.29677$

$-9.26421$

$-9.47482$

$-9.04892$

$-8.77005$

$-9.97999$

$-10.43551$

$-11.20547$

$-11.02026$

$-10.05454$

$-10.71282$

$-11.17575$ sd

0.75707

0.66080

0.60892

0.54894

0.56093

0.56250

0.61827

0.58350

0.59324

0.57537

0.56460

0.55538

0.55403

0.56484

0.54403

0.57513

0.57769

0.56766

0.63312

0.65802

0.54550

0.60533

0.55152

0.59530

0.57851

0.61015

0.57825

0.76492

0.56482

0.59345

0.56343

0.54960

0.64693

0.65552

0.64058

0.56976

1.35393

0.80637

0.88832

1.24704

1.14974

1.54853
0.73266
Deposition Rate

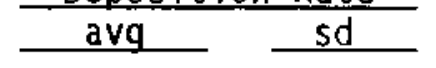

$-18.53714$

$-18.38347$

$-18.28591$

$-18.03664$

$-18.00339$

$-18.11183$

$-18.14247$

$-17.90916$

$-17.71238$

$-17.54245$

$-17.10221$

$-16.47021$

$-16.93104$

$-17.05292$

$-17.42392$

$-17.57588$

$-16.72695$

$-17.56954$

$-17.06249$

$-17.22802$

$-17.34093$

$-16.85854$

$-16.78238$

$-16.56260$

$-18.91921$

$-18.63931$

$-18.27562$

$-18.36280$

$-18.52713$

$-17.94001$

$-18.30019$

$-17.72465$

$-17.69209$

$-17.90269$

$-17.47680$

$-17.19793$

$-18.40787$

$-18.79257$

$-19.63334$

$-19.44814$

$-18.48242$

$-19.14069$

$-19.45255$
0.75706

0.66069

0.60894

0.54884

0.56083

0.56241

0.61819

0.58361

0.59328

0.57531

0.56458

0.73273

0.55542

0.55402

0.56479

0.54398

0.57510

0.57756

0.56765

0.63321

0.65803

0.54547

0.60535

0.55148

0.59538

0.57841

0.61007

0.57835

0.76498

0.56482

0.59346

0.56349

0.54970

0.64697

0.65550

0.64055

0.56977

1.19416

0.80635

0.88827

1.24708

1. 14975

1.36704
Surface Contamination. avg sd

$-12.67415$

$-12.44985$

$-12.22258$

$-12.05383$

$-12.12573$

$-12.23115$

$-12.26712$

$-12.04960$

$-11.85515$

$-11.59895$

$-11.10646$

$-10.50607$

$-10.94883$

$-11.07089$

$-11.46939$

$-11.63879$

$-10.75201$

$-11.59173$

$-11.06154$

$-11.32293$

$-11.42265$

$-10.89550$

$-10.83184$

$-10.62968$

$-12.95552$

$-12.66103$

$-12.22608$

$-12.42271$

$-12.60165$

$-11.91568$

$-12.28172$

$-11.67985$

$-11.67943$

$-11.97399$

$-11.54730$

$-11.24972$

$-12.46046$

$-12.91027$

$-13.72044$

$-13.55620$

$-12.59476$

$-13.27632$

$-13.77924$

0.90139

0.77091

0.62521

0.58968

0.64484

0.65476

0.72027

0.66333

0.66842

0.62582

0.60872

0.74613

0.56986

0.56835 
IABLE 9. (contd)

Time-Integrated

Census Div.

KL1 $\quad-10.41174$

KL2 -10.26250

KL3 - 11.29418

KL4 -11.27730

WA1 -9.12124

WA2 $\quad-8.68905$

WA3 $\quad-8.60954$

WA4 $\quad-8.75493$

WA5 $\quad-8.86089$

WA6 $\quad-9.02489$

WA7 $\quad-9.09045$

WA8 -8.95154

WA9 $\quad-8.88149$

WAlO $\quad-8.92473$

YA1 -10.02599

YA2 -11.28012

YA5 -11.21776

YA6 $\quad-9.55887$

YA7 -10.40841

YA9 -9.05593

YA10 -9.44041

YAI1 -10.25143

M01 $\quad-9.50660$

M02 -9.56320

$\mathrm{MO3} \quad-9.68731$

UM1 $\quad-9.09286$

UM2 $\quad-8.95802$

UM3 $\quad-9.23139$

UM4 $\quad-9.08684$

UM5 $\quad-9.06778$

UM6 $\quad-9.24121$

UM7 $\quad-9.44064$

UM8 $\quad-9.29372$

UM9 $\quad-9.32496$

UM10 $\quad-9.44094$

UMI $1 \quad-8.93054$

UMI $2-9.23178$

UMI3 - $\quad-9.05847$

UM1 $4 \quad-9.24765$ ation

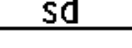

1.03890

0.87255

1.10065

1.04467

0.55919

0.53565

0.55202

0.53125

0.52628

0.52338

0.51748

0.51832

0.53123

0.51885

1.06604

1.59820

1.35431

0.93483

I. 02767

0.56614

0.60751

0.93418

0.54368

0.53948

0.54974

0.55161

0.53479

0.54478

0.55136

0.54766

0.55492

0.55486

0.54004

0.54292

0.54794

0.54194

0.54907

0.53034

0.54432
Deposition Rate

$-18.83961$

$-18.69037$

$-19.72205$

$-19.70518$

$-17.54912$

$-17.11693$

$-17.03741$

$-17.18280$

$-17.28877$

$-17.45276$

$-17.51833$

$-17.37942$

$-17.30937$

$-17.35261$

$-18.45387$

$-19.60599$

$-19.64564$

$-17.98675$

$-18.83629$

$-17.48381$

$-17.86829$

$-18.67931$

$-17.93448$

$-17.99108$

$-18.11519$

$-17.52074$

$-17.38589$

$-17.65927$

$-17.51471$

$-17.49566$

$-17.66909$

$-17.86852$

$-17.72160$

$-17.75283$

$-17.86881$

$-17.35842$

$-17.65966$

$-17.48635$

$-17.67553$
1.03890

0.87253

1.10060

1.04458

0.55920

0.53559

0.55203

0.53130

0.52619

0.52334

0.51742

0.51827

0.53113

0.51877

1.06602

1.49121

1.35428

0.93487

1.02773

0.56601

0.60749

0.93422

0.54362

0.53936

0.54968

0.55159

0.53473

0.54485

0.55148

0.54754

0.55476

0.55495

0.54001

0.54297

0.54806

0.54179

0.54912

0.53023

0.54432
Surface Contamination

avg sd

$-12.91897$

1.13942

$-12.73501 \quad 1.00537$

$-13.77451 \quad 1.22354$

$-13.80407$

1.12332

$-11.58961$

$-11.17586$

$-11.11335$

$-11.26758$

$-11.32851$

$-11.49861$

$-11.55864$

$-11.42332$

$-11.39862$

$-11.40072$

$-12.56759$

$-13.91284$

$-13.80456$

$-12.03473$

$-12.94437$

$-11.53472$

$-11.96331$

$-12.79749$

$-11.96625$

$-11.98695$

$-12.12731$

$-11.58776$

$-11.45248$

$-11.68556$

$-11.54731$

$-11.56244$

$-11.68225$

$-11.88716$

$-11.75905$

$-11.76765$

$-11.93408$

$-11.44395$

$-11.67842$

$-11.53820$

$-11.73707$
0.61103

0.54925

0.55702

0.53656

0.54327

0.54568

0.52959

0.53134

0.53680

0.53518

1.10310

1.55628

1. 33755

0.97804

1.06125

0.60385

0.69123

1.03374

0.60388

0.60361

0.61169

0.53526

0.53925

0.61435

0.57333

0.54007

0.57583

0.61256

0.53900

0.61389

0.54571

0.54137

0.61169

0.53439

0.53955 
IABLE 10. Computational Results for August. A]l numerical values are logarithms to base 10 . Units are as follows: time-integrated air concentration, $\left[(\mathrm{Ci}-\mathrm{s}) / \mathrm{m}_{2}^{3}\right] / \mathrm{Ci}$; deposition rate, $\left[\left(\mathrm{Ci} / \mathrm{m}^{2}\right) / \mathrm{s}\right] / \mathrm{Ci}$; surface contamination, $\left(\mathrm{Ci} / \mathrm{m}^{2}\right) / \mathrm{Ci}$.

Time-Integrated

Census Air Concentration

\begin{tabular}{|c|c|c|c|}
\hline & & & \\
\hline Div. & avg & sd & avg \\
\hline $\mathrm{ADl}$ & -10.03478 & 0.74988 & -18.46266 \\
\hline $\mathrm{AD2}$ & -9.90701 & 0.76774 & -18.33488 \\
\hline $\mathrm{AD3}$ & -9.84858 & 0.74904 & -18.27646 \\
\hline AD4 & -9.62929 & 0.67263 & -18.05717 \\
\hline AD5 & -9.59844 & 0.62957 & -18.02633 \\
\hline AD6 & -9.70834 & 0.61401 & -18.13622 \\
\hline AD7 & -9.75157 & 0.59754 & -18.17945 \\
\hline AD8 & -9.58488 & 0.59153 & -18.01276 \\
\hline AD9 & -9.31937 & 0.57270 & -17.74725 \\
\hline AD10 & -9.15006 & 0.59420 & -17.57793 \\
\hline $\mathrm{BE} 1$ & -8.67512 & 0.57554 & -17.10299 \\
\hline BE2 & -7.96541 & 0.74689 & -16.39329 \\
\hline BE3 & -8.54461 & 0.55973 & -16.97249 \\
\hline BE4 & -8.67809 & 0.57125 & -17.10597 \\
\hline BE 5 & -9.16598 & 0.65462 & -17.59385 \\
\hline BE6 & -9.43766 & 0.67570 & -17.86553 \\
\hline BE7 & -8.29735 & 0.59097 & -16.72523 \\
\hline BE8 & -9.38255 & 0.80088 & -17.81042 \\
\hline BE9 & -8.65889 & 0.58821 & -17.08677 \\
\hline FRI & -8.74676 & 0.61345 & -17.17464 \\
\hline FR2 & -8.92627 & 0.68843 & -17.35414 \\
\hline FR3 & -8.43227 & 0.55034 & -16.86014 \\
\hline FR4 & -8.25258 & 0.60250 & -16.68046 \\
\hline FR5 & -8.05730 & 0.56017 & -16.48518 \\
\hline GR2 & -10.93222 & 1.38730 & -19.36009 \\
\hline GR3 & -10.30991 & 0.83026 & -18.73779 \\
\hline GR4 & -9.91215 & 0.72719 & -18.34002 \\
\hline GR5 & -9.88265 & 0.78524 & -18.31053 \\
\hline GR6 & 96053 & 0.89033 & -18.38840 \\
\hline GR7 & 46527 & 0.68210 & -17.89314 \\
\hline GR8 & 1921 & 0.75130 & -18.24708 \\
\hline GR9 & 912 & 0.65776 & -17.86699 \\
\hline GRIO & 2110 & 0.62994 & -17.84898 \\
\hline GR11 & 32130 & 0.63507 & -17.74917 \\
\hline GR12 & 91625 & 0.57671 & -17.34413 \\
\hline GR13 & -8.60563 & 0.64525 & -17.03351 \\
\hline GR14 & -9.93558 & 0.79177 & -18.36346 \\
\hline KIl & -10.34188 & 1.25795 & -18.76975 \\
\hline $\mathrm{K} 12$ & -11.77901 & 1.23631 & -20.20689 \\
\hline $\mathrm{KI} 7$ & -11.51279 & 1.30142 & -19.94066 \\
\hline $\mathrm{KI}$ & -9.77443 & 0.97262 & -18.20231 \\
\hline $\mathrm{KIg}$ & -10.84101 & 1.11161 & -19.26888 \\
\hline KI10 & -11.14444 & 1.18880 & -19.57232 \\
\hline
\end{tabular}

Surface Contamination avg sd

0.74987

0.76781

0.74904

0.67262

0.62944

0.61401

0.59758

0.59142

0.57271

0.59420

0.57555

0.74683

0.55977

0.57119

0.65457

0.67563

0.59091

0.80092

0.58824

0.61343

0.68847

0.55037

0.60249

0.56015

1.38734

0.83022

0.72725

0.78528

0.89036

0.68219

0.75138

0.65784

0.62988

0.63514

0.57664

0.64520

0.79178

1.25795

1. 23627

1. 30144

0.97255

1. 11165

1. 18877
$-12.61365$

$-12.47548$

$-12.39754$

$-12.18108$

$-12.19873$

$-12.32232$

$-12.33311$

$-12.20998$

$-11.93432$

$-11.72450$

$-11.10994$

$-10.41249$

$-10.92953$

$-11.06271$

$-11.64886$

$-11.93927$

$-10.69603$

$-11.84527$

$-11.09002$

$-11.29860$

$-11.45609$

$-10.80613$

$-10.73488$

$-10.46535$

$-13.52758$

$-12.91621$

$-12.52994$

$-12.44943$

$-12.49575$

$-12.05117$

$-12.44158$

$-12.02098$

$-11.99169$

$-11.87576$

$-11.44287$

$-11.04066$

$-12.49851$

$-12.77499$

$-14.27081$

$-13.97411$

$-12.14620$

$-13.25835$

$-13.53316$
0.80420

0.79438

0.77418

0.69845

0.65635

0.64476

0.63715

0.62348

0.59712

0.62239

0.58200

0.75293

0.56539

0.57737

0.72451

0.73742

0.60316

0.84838

0.60402

0.64560

0.73369

0.56129

0.61719

0.55619

1. 39173

0.82000

0.73904

0.79668

0.95821

0.72004

0.76138

0.68825

0.64808

0.67303

0.59806

0.63796

0.80328

1.23947

1.18089

1.27416

0.96299

1. 10929

1. 19262 
IABLE 10. (contd)

Time-Integrated

Census Air Concentration$$
\text { . }
$$

$K L 1 \quad-10.93472$

KL2 -10.80101

KL3 -11.56307

KL4 -11.04965

WA1 -9.15062

WA2 -8.70772

WA3 $\quad-8.60962$

WA4 -8.79722

WA5 -8.95585

WA6 $\quad-9.09994$

WA7 -9.12486

WA8 $\quad-9.04273$

WA9 $\quad-8.96798$

WA10 -9.02768

YAl -10.19236

YA2 -11.50527

YA5 -11.72412

YA6

YA7

YA9

YA10

YAIl

M01

MO2

$\mathrm{MO3}$

UM1

UM2

UM3

UM4

UM5

UM6

UM7

UM8

UM9

UM10

UM1 1

UM12

UM13

UM1 4
$-9.79933$

$-11.00876$

$-9.33259$

$-10.11668$

$-10.86813$

$-10.13858$

$-9.96258$

$-10.37088$

$-9.10627$

$-9.05323$

$-9.43466$

$-9.20804$

$-9.12899$

$-9.36801$

$-9.65387$

$-9.30187$

$-9.52957$

$-9.47027$

$-9.00851$

$-9.39445$

$-9.17190$

$-9.32357$ sd

1.40205

1. 38842

1.42087

0.90292

0.55830

0.53981

0.55268

0.53320

0.54694

0.55437

0.54715

0.53276

0.53262

0.53551

1.38980

1.82002

2.17512

1. 26798

1.57360

0.65189

1.20364

1.44142

1.28065

0.86893

1.34294

0.54218

0.53299

0.59386

0.57158

0.53388

0.56228

0.60539

0.53598

0.61024

0.54348

0.53668

0.59586

0.52973

0.54071
Deposition Rate avg

$-19.30975$

$-19.22888$

$-19.99094$

$-19.47753$

$-17.57850$

$-17.13560$

$-17.03749$

$-17.22510$

$-17.38373$

$-17.52781$

$-17.55273$

$-17.47061$

$-17.39586$

$-17.45555$

$-18.62024$

$-19.81801$

$-19.63774$

$-18.22721$

$-19.37271$

$-17.76047$

$-18.54455$

$-19.24974$

$-18.56646$

$-18.39045$

$-18.79875$

$-17.53415$

$-17.48111$

$-17.86254$

$-17.63592$

$-17.55687$

$-17.79589$

$-18.08175$

$-17.72974$

$-17.95745$

$-17.89814$

$-17.43639$

$-17.82232$

$-17.59978$

$-17.75145$

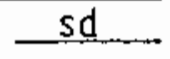

1.33642

1.38845

1.42087

0.90290

0.55822

0.53979

0.55264

0.53316

0.54695

0.55440

0.54712

0.53276

0.53268

0.53547

1.38982

1.71084

1.77176

1.26796

1.49407

0.65181

1. 20367

1. 38145

1.28065

0.86896

1.34302

0.54210

0.53299

0.59387

0.57164

0.53382

0.56225

0.60532

0.53599

0.61024

0.54348

0.53673

0.59587

0.52972

0.54069
Surface Contamination

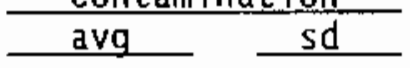

$-13.33763$

1.54724

$-13.19401$

$-14.02113$

$-13.31654$

$-11.62975$

$-11.19033$

$-11.02506$

$-11.24140$

$-11.45631$

$-11.60293$

$-11.63863$

$-11.52668$

$-11.41403$

$-11.50929$

$-12.54195$

$-13.84399$

$-14.12535$

$-12.23691$

$-13.40540$

$-11.81641$

$-12.61955$

$-13.26046$

$-12.54675$

$-12.38577$

$-12.77463$

$-11.52407$

$-11.50775$

$-11.88800$

$-11.60728$

$-11.56939$

$-11.75337$

$-12.06917$

$-11.69150$

$-11.98726$

$-11.84204$

$-11.45106$

$-11.87353$

$-11.62560$

$-11.75880$
1.47972

1.71289

0.85119

0.59666

0.55420

0.55462

0.53946

0.55867

0.56794

0.56086

0.53870

0.53265

0.54302

1. 33171

1.79655

2. 22782

1.70540

0.79618

1. 34478

1.57524

1.27066

0.89119

1. 36371

0.53610

0.53193

0.62535

0.56653

0.52768

0.56179

0.63108

0.52725

0.64861

0.53393

0.53439

0.63103

0.52483

0.53386
1. 32229 
TABLE 11. Computational Results for September. All numerical values are logarithms to base 10. Units are as follows: time-integrated air concentration, $\left[(\mathrm{Ci}-\mathrm{s}) / \mathrm{m}_{2}^{3}\right] / \mathrm{Ci}$; deposition rate, $\left[\left(\mathrm{Ci} / \mathrm{m}^{2}\right) / \mathrm{s}\right] / \mathrm{C} ;$; surface contamination, $\left(\mathrm{Ci} / \mathrm{m}^{2}\right) / \mathrm{Ci}$.

Time-Integrated

Census Div

AD1 1

AD3

AD4

AD5

AD6

AD7

AD8

AD9

AD10

BE1

BE2

BE3

BE4

BE5

BE6

BE7

BE8

BE9

FR1

FR2

FR3

FR4

FR5

GR2

GR3

GR4

GR5

GR6

GR7

GR8

GR9

GRIO

GRI1

GRI2

GR13

GR14

KII

$\mathrm{KI} 2$

KI7

KI8

K19

KI10
Air Concentration

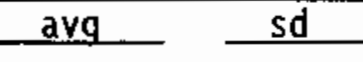

$-10.03370$

$-9.89559$

$-9.83184$

$-9.61199$

$-9.57696$

$-9.65336$

$-9.71959$

$-9.52258$

$-9.20510$

$-9.01586$

$-8.60852$

$-7.93729$

$-8.35265$

$-8.48471$

$-9.18904$

$-9.49304$

$-8.13596$

$-9.19664$

$-8.42936$

$-8.64622$

$-8.94600$

$-8.32953$

$-8.23861$

$-8.13486$

$-10.39850$

$-9.99288$

$-9.72884$

$-9.65674$

$-10.06521$

$-9.39406$

$-9.60497$

$-9.32862$

$-9.27851$

$-9.47337$

$-9.05620$

$-8.75263$

$-9.69898$

$-11.43564$

$-13.60965$

$-12.85471$

$-10.53504$

$-12.03793$

$-11.94029$
0.59730

0.60426

0.60826

0.60519

0.59765

0.56653

0.54527

0.54001

0.56445

0.59975

0.58439

0.75949

0.52427

0.53193

0.66708

0.68418 .

0.53342

0.73419

0.56331

0.63499

0.63370

0.53147

0.57390

0.55288

0.77920

0.62737

0.61458

0.58322

0.77099

0.57713

0.58434

0.62853

0.76892

0.75583

0.76824

0.57450

1.47957

1.02465

1.01367

1.51705

1.52712

1.62071
0.62078
Deposition Rate

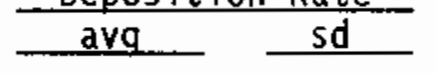

$-18.44734$

$-18.30923$

$-18.24547$

$-18.02563$

$-17.99060$

$-18.06700$

$-18.13323$

$-17.93621$

$-17.61874$

$-17.42949$

$-17.02216$

$-16.35093$

$-16.76628$

$-16.89835$

$-17.60267$

$-17.90668$

$-16.54959$

$-17.61028$

$-16.84300$

$-17.05986$

$-17.35963$

$-16.74317$

$-16.65224$

$-16.54849$

$-18.81213$

$-18.40652$

$-18.14248$

$-18.07037$

$-18.47884$

$-17.80770$

$-18.01861$

$-17.74226$

$-17.69215$

$-17.88701$

$-17.46984$

$-17.16627$

$-18.11262$

$-19.84927$

$-21.80502$

$-21.26834$

$-18.88523$

$-20.22091$

$-19.92811$
0.59728

0.60406

0.60829

0.60517

0.59758

0.56649

0.54522

0.53999

0.56441

0.59978

0.58429

0.75954

0.52433

0.53181

0.66706

0.68418

0.53348

0.73424

0.56314

0.63490

0.63377

0.53150

0.57401

0.55280

0.77921

0.62733

0.61458

0.58314

0.77108

0.57717

0.58426

0.62078

0.62850

0.76880

0.75577

0.76830

0.57436

1.47956

0.85841

1.01370

1.42869

1.31260

1.14591

Surface Contamination

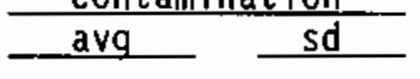

$-12.66057$

0.76392

$\begin{array}{ll}-12.52717 & 0.74699\end{array}$

$\begin{array}{ll}-12.43108 & 0.74914\end{array}$

$-12.20340 \quad 0.69681$

$\begin{array}{ll}-12.15967 & 0.67602\end{array}$

$\begin{array}{ll}-12.21693 & 0.64809\end{array}$

$\begin{array}{ll}-12.31123 & 0.63287\end{array}$

$\begin{array}{ll}-12.08110 & 0.57773\end{array}$

$\begin{array}{ll}-11.77872 & 0.60424\end{array}$

$\begin{array}{ll}-11.54872 & 0.65685\end{array}$

$\begin{array}{ll}-11.05773 & 0.60111\end{array}$

$-10.39943 \quad 0.77425$

$\begin{array}{ll}-10.78401 & 0.56288\end{array}$

$-10.91866 \quad 0.56825$

$-11.69591$

0.74018

$-12.02792$

0.78094

$-10.57360$

0.56107

$-11.66161$

$-10.83127$

0.84255

0.59106

0.67062

$-11.22189$

0.67346

$\begin{array}{ll}-10.78544 & 0.57003\end{array}$

$-10.78726$

$-10.57933$

0.61543

0.57645

$\begin{array}{ll}-13.02504 & 0.97382\end{array}$

$\begin{array}{ll}-12.58062 & 0.78252\end{array}$

$\begin{array}{ll}-12.27523 & 0.74649\end{array}$

$\begin{array}{ll}-12.19864 & 0.61598\end{array}$

$\begin{array}{ll}-12.70027 & 0.81688\end{array}$

$\begin{array}{lll}-11.91190 & 0.63337\end{array}$

$\begin{array}{ll}-12.14477 & 0.66347\end{array}$

$\begin{array}{ll}-11.83367 & 0.69087\end{array}$

$\begin{array}{ll}-11.81099 & 0.68996\end{array}$

$-11.99042$

0.76230

$-11.51715$

0.74585

$-11.20559$

0.77294

$-12.25073$

0.61348

$-14.12341$

1.47726

1.19078

0.97969

$-15.73962$

1.49316

1.35777

$-14.80910$

1.43383 
IABLE 11. (contd)

Time-Integrated

Census Air Concentration

Div.

KL2

$\mathrm{KL} 3$

KL4

WAl

WA2

WA3

WA4

WA5

WA6

WA7

WA8

WA9

WA10

YAl

YA2

YA5

YA6

YA7

YA9

YAIO

YA11

MO1

$\mathrm{MO2}$

$\mathrm{MO3}$

UM1

UM2

UM3

UM4

UM5

UM6

UM7

UM8

UM9

UM10

UMI1

UM12

UM13

UM14

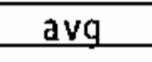

$-11.01033$

$-10.70608$

$-11.77687$

$-12.02468$

$-9.26429$

$-8.87439$

$-8.70632$

$-8.95063$

$-9.10334$

$-9.25168$

$-9.27545$

$-9.16583$

$-9.12408$

$-9.15933$

$-10.65573$

$-11.96837$

$-12.75572$

$-10.36148$

$-11.69125$

$-9.71144$

$-10.26256$

$-11.20398$

$-9.80004$

$-9.71867$

$-9.96024$

$-9.13603$

$-9.18026$

$-9.12888$

$-8.93732$

$-9.22669$

$-9.07464$

$-9.39404$

$-9.23119$

$-9.32211$

$-9.43748$

$-9.15257$

$-9.14602$

$-9.22763$

$-9.33583$

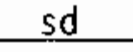

1.24397

1.05398

1.24345

1.40978

0.54828

0.56093

0.54525

0.54881

0.55969

0.56068

0.55365

0.54657

0.55638

0.54968

1.50006

1.69500

1.72614

1.63827

1.84712

0.98484

0.93281

1.56486

0.65077

0.56706

0.68778

0.54582

0.55346

0.56872

0.52520

0.55360

0.52349

0.54289

0.53436

0.54878

0.54515

0.55850

0.56835

0.53996

0.54788
Deposition Rate

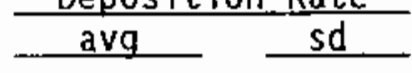

$-19.42397$

$-19.11972$

$-20.19050$

$-20.32678$

$-17.67793$

$-17.28803$

$-17.11996$

$-17.36427$

$-17.51697$

$-17.66531$

$-17.68909$

$-17.57947$

$-17.53772$

$-17.57297$

$-19.06936$

$-20.06936$

$-20.86862$

$-18.77512$

$-19.97781$

$-18.12507$

$-18.67620$

$-19.54592$

$-18.21367$

$-18.13230$

$-18.37388$

$-17.54967$

$-17.59390$

$-17.54252$

$-17.35096$

$-17.64032$

$-17.48827$

$-17.80767$

$-17.64482$

$-17.73575$

$-17.85112$

$-17.56620$

$-17.55966$

$-17.64126$

$-17.74946$
1. 24398

1.05400

1.24344

1.32434

0.54840

0.56092

0.54513

0.54882

0.55966

0.56067

0.55366

0.54645

0.55633

0.54965

1.50002

1.41973

1.61704

1.63826

1.77273

0.98485

0.93284

1.49674

0.65080

0.56703

0.68783

0.54578

0.55344

0.56872

0.52520

0.55358

0.52346

0.54289

0.53451

0.54879

0.54519

0.55843

0.56848

0.53996

0.54787
Surface Contamination

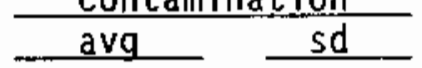

$-13.59070$

1.39801

$-13.28105$

$-14.36506$

1.25732

1.44043

1.49517

0.57765

0.60635

0.57954

0.59983

0.60068

0.59802

0.60112

0.59693

0.61255

0.59866

1.57007

1.60157

1.73198

1.75870

2.01525

1.06788

1.10429

1.74356

0.83084

0.67121

0.87949

0.59280

0.60864

0.60097

0.53788

0.61212

0.53748

0.58547

0.56173

0.59716

0.57916

0.61366

0.59764

0.59418

0.60404 
IABLE 12. Computational Results for October. A11 numerical values are logarithms to base 10. Units are as follows: time-integrated air concentration, $\left[(\mathrm{Ci}-\mathrm{s}) / \mathrm{m}_{2}^{3}\right] / \mathrm{C} i$; deposition rate, $\left[\left(\mathrm{Ci} / \mathrm{m}^{2}\right) / \mathrm{s}\right] / \mathrm{Ci}$; surface contamination, $\left(\mathrm{C} i / \mathrm{m}^{2}\right) / \mathrm{C} \mathrm{i}$.

Time-Integrated

Census Air Concentration

Div.

AD1

$\mathrm{AD} 2$

$\mathrm{AD} 3$

AD4

AD5

AD6

AD7

AD8

AD9

AD10

BEl

BE2

$8 \mathrm{E} 3$

BE4

$8 \mathrm{E} 5$

BE6

BE7

BE8

BE9

FR1

FR2

FR3

FR4

FR5

GR2

GR3

GR4

GR5

GR6

GR7

GR8

GR9

GR10

GRI1

GR12

GR13

GR14

KII

$\mathrm{KI} 2$

KI7

KI8

KI9

KIIO

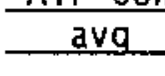

$-10.29458$

$-10.18947$

$-9.95914$

$-9.61082$

$-9.68594$

$-9.82394$

$-9.92681$

$-9.74052$

$-9.35443$

$-9.07822$

$-8.74189$

$-7.81448$

$-8.40404$

$-8.56303$

$-9.79493$

$-10.48093$

$-8.13196$

$-9.69525$

$-8.48968$

$-8.74906$

$-9.04807$

$-8.31602$

$-8.20772$

$-8.02711$

$-10.88496$

$-10.58489$

$-9.90024$

$-9.74084$

$-9.98496$

$-9.36352$

$-9.74456$

$-9.23938$

$-9.22020$

$-9.28017$

$-8.83235$

$-8.47484$

$-9.78330$

$-11.07494$

$-11.69934$

$-11.74746$

$-10.71280$

$-12.31832$

$-12.46147$

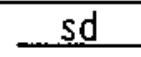

0.93590

0.87821

0.85211

0.58539

0.60276

0.66355

0.70183

0.65688

0.59577

0.58822

0.74711

0.73497

0.56734

0.57292

1.52108

1.73100

0.57408

1.30010

0.60699

0.71448

0.74453

0.54560

0.62407

0.54747

1.66073

1.30451

0.83937

0.75775

0.86055

0.58305

0.68284

0.57117

0.56474

0.62672

0.59598

0.70119

0.73648

1.69661

0.72519

1.04967

1.70265

1.59306

1.44973
Deposition Rate

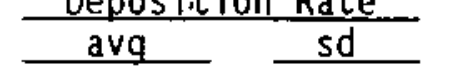

$-18.72246$

$-18.61735$

$-18.38701$

$-18.03870$

$-18.11381$

$-18.25182$

$-18.35468$

$-18.16839$

$-17.78231$

$-17.50609$

$-17.16976$

$-16.24236$

$-16.83192$

$-16.99092$

$-18.04979$

$-18.60015$

$-16.55983$

$-18.12313$

$-16.91755$

$-17.17693$

$-17.47595$

$-16.74389$

$-16.63560$

$-16.45498$

$-18.87702$

$-19.01277$

$-18.32811$

$-18.16872$

$-18.41284$

$-17.79140$

$-18.17243$

$-17.66725$

$-17.64808$

$-17.70804$

$-17.26023$

$-16.90273$

$-18.21117$

$-19.23440$

$-20.12722$

$-20.17533$

$-19.02840$

$-20.54031$

$-20.88935$
0.93588

0.87821

0.85211

0.58530

0.60285

0.66348

0.70184

0.65685

0.59585

0.58837

0.74710

0.73497

0.56735

0.57284

1.21521

1.31030

0.57404

1.30006

0.60696

0.71453

0.74454

0.54547

0.62411

0.54737

0.83872

1.30452

0.83939

0.75769

0.86054

0.58303

0.68293

0.57103

0.56466

0.62675

0.59608

0.70110

0.73648

1.35966

0.72523

1.04967

1.56489

1.43892

1.44969
Surface Contamination

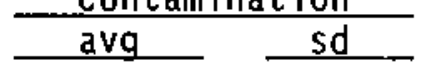

$-12.88581$

$-12.75698$

$-12.38328$

$-12.09159$

$-12.23621$

$-12.42772$

$-12.51334$

$-12.32288$

$-11.86950$

$-11.58834$

$-11.27044$

$-10.30109$

$-10.84888$

$-11.02186$

$-12.38405$

$-13.07184$

$-10.58977$

$-12.24869$

$-10.93749$

$-11.25085$

$-11.56545$

$-10.77915$

$-10.70786$

$-10.52179$

$-13.29888$

$-13.03027$

$-12.32502$

$-12.30727$

$-12.50757$

$-11.86055$

$-12.24260$

$-11.70142$

$-11.71115$

$-11.85401$

$-11.39583$

$-10.96422$

$-12.33791$

$-13.73214$

$-14.24685$

$-14.33627$

$-13.31128$

$-14.98238$

$-15.08179$

1.11304

1.00196

0.94036

0.62821

0.67301

0.79690

0.84441

0.76948

0.66468

0.64394

0.76030

0.76217

0.57120 
TABLE 12. (contd)

Time-Integrated

Census Air Concentration

KL1 -11.30635

KL2 -11.56738

KL3 $\quad-11.69079$

KL4 -10.65725

HA1 -9.34060

WA2 -8.78088

WA3 -8.60187

WA4 -8.86457

WA5 $\quad-9.07549$

WA6 $\quad-9.30520$

WA7 $\quad-9.38316$

WA8 -9.19779

WA9 $\quad-9.03168$

WA10 -9.16253

YAl $\quad-10.77780$

YA2 -11.82322

YA5 -11.19227

YA6 -10.11701

YA7 -11.39876

YA9 -10.59034

YA10 -11.25584

YA11 - 11.10571

MO1 $\quad-10.77716$

M02 -10.48980

M03 -10.95273

UMl $\quad-9.08601$

UM2 $\quad-9.11131$

UM3 $\quad-9.53977$

UM4 $\quad-9.09244$

UM5 - -9.14951

UM6 -9.28365

UM7 $\quad-9.97423$

UM8 $\quad-9.35442$

UM9 $\quad-9.84066$

UM10 $\quad-9.51780$

UM1 $1 \quad-9.05789$

UM12 -9.51486

UM13 -9.20829

UM14 -9.29736

sd

1.78383

1.85413

1.78336

0.66257

0.60181

0.53846

0.53838

0.53026

0.54026

0.55664

0.58000

0.54513

0.53825

0.54122

1.74542

1.32414

0.54599

1. 34843

1.97122

2.03431

1.96851

1.82818

1.66654

1.35560

1.82055

0.54350

0.53859

0.74898

0.56094

0.54094

0.56470

0.92373

0.55151

0.90942

0.58092

0.53907

0.74079

0.54822

0.55620

Deposition Rate

$-19.45408$

$-19.66384$

$-19.95723$

$-19.08512$

$-17.76847$

$-17.20876$

$-17.02975$

$-17.29245$

$-17.50337$

$-17.73308$

$-17.81103$

$-17.62567$

$-17.45955$

$-17.59041$

$-19.02288$

$-20.25110$

$-19.62015$

$-18.54488$

$-19.50024$

$-18.47484$

$-19.16872$

$-19.36517$

$-18.97677$

$-18.91768$

$-19.02937$

$-17.51389$

$-17.53919$

$-17.96764$

$-17.52031$

$-17.57739$

$-17.71153$

$-18.40211$

$-17.78230$

$-18.26854$

$-17.94568$

$-17.48577$

$-17.94273$

$-17.63617$

$-17.72523$

\subsection{3}

1.48907

1.68160

0.66251

0.60176

0.53846

0.53835

0.53021

0.54035

0.55674

0.58001

0.54519

0.53826

0.54117

1.51419

1.32414

0.54592

1. 34839

1. 58936

1.26725

1.36316

1.66317

1. 36030

1. 35561

1. 34572

0.54345

0.53854

0.74896

0.56100

0.54081

0.56471

0.92372

0.55148

0.90939

0.58083

0.53894

0.74076

0.54815

0.55624
Surface Contamination

avq sd

$-14.02171$

$-14.28724$

$-14.47779$

$-13.50324$

$-11.88480$

$-11.31333$

$-11.10163$

$-11.36952$

$-11.61515$

$-11.86513$

$-11.97600$

$-11.75270$

$-11.54803$

$-11.70624$

$-13.39392$

$-14.55771$

$-14.01979$

$-12.85191$

$-14.11390$

$-13.24597$

$-13.87608$

$-13.93492$

$-13.40615$

$-13.05186$

$-13.58910$

$-11.57697$

$-11.64285$

$-12.04542$

$-11.53362$

$-11.67647$

$-11.73189$

$-12.46530$

$-11.81138$

$-12.37446$

$-11.98573$

$-11.57384$

$-12.03999$

$-11.76809$

$-11.80979$
1.65581

1.74514

1.69887

0.64356

0.62195

0.55677

0.54582

0.53678

0.55140

0.56599

0.58766

0.55734

0.55041

0.55651

1.76371

1.19758

0.54207

1.39192

1.72304

1.92872

1.84396

1.79773

1.56879

1.29366

1.71175

0.54205

0.55516

0.79314

0.56626

0.55137

0.56603

0.89685

0.55097

0.91886

0.57032

0.54664

0.78844

0.57736

0.57940 
TABLE 13. Computational Results for November. All numerical values are logarithms to base 10. Units are as follows: time-integrated air concentration, $\left[(\mathrm{Ci}-\mathrm{s}) / \mathrm{m}_{2}^{3}\right] / \mathrm{C}$; deposition rate, $\left[\left(\mathrm{Ci} / \mathrm{m}^{2}\right) / \mathrm{s}\right] / \mathrm{Ci}$; surface contamination, $\left(\mathrm{Ci} / \mathrm{m}^{2}\right) / \mathrm{Ci}$.

Time-Integrated

Census Air Concentration

Div. avg sd

AD1 $\quad-9.94455$

$A D 2$

AD3

AD4

AD5

AD6

AD7

AD8

AD 9

AD10

BE 1

BE2

BE3

BE4

BE5

BE6

BE7

BE8

BE9

FR1

FR2

FR3

FR4

FR5

GR2

GR3

GR4

GR5

GR6

GR7

GR8

GR9

GR10

GR1 I

GR12

GR13

GR14

KII

KI2

KI7

KI8

KI9

KIIO
$-9.83862$

$-9.55941$

$-9.41473$

$-9.52312$

$-9.62607$

$-9.76029$

$-9.59226$

$-9.21943$

$-8.86322$

$-8.68726$

$-7.81664$

$-8.31117$

$-8.43026$

$-9.44995$

$-9.83938$

$-8.07234$

$-9.44736$

$-8.40690$

$-8.69182$

$-9.21425$

$-8.26115$

$-8.39668$

$-8.14893$

$-9.98362$

$-9.88465$

$-9.57287$

$-9.66232$

$-9.95139$

$-9.17323$

$-9.50506$

$-9.09185$

$-9.02744$

$-9.45673$

$-9.24138$

$-8.64605$

$-9.69454$

$-10.20648$

$-10.82926$

$-11.70478$

$-10.53273$

$-11.35261$

$-11.42855$
0.54477

0.55570

0.54836

0.53932

0.55549

0.53769

0.59982

0.57913

0.54688

0.57639

0.64245

0.76418

0.53940

0.52776

0.91870

0.66330

0.54884

0.87807

0.61834

0.67096

0.73077

0.53582

0.61177

0.56749

0.70808

0.76293

0.76063

0.71287

1.33461

0.56153

0.73483

0.56459

0.54412

1.34556

1.51014

0.90765

0.71588

1. 36656

0.88438

1.81061

1.70074

1.32610

1.85668
Deposition Rate

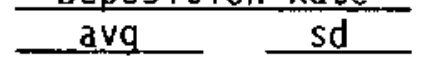

$-18.35818$

$-18.25225$

$-17.97305$

$-17.82837$

$-17.93676$

.18 .03971

$-18.17392$

$-18.00590$

$-17.63307$

$-17.27686$

$-17.10089$

$-16.23028$

$-16.72481$

$-16.84389$

$-17.86358$

$-18.25302$

$-16.48597$

$-17.86099$

$-16.82053$

$-17.10545$

$-17.62789$

$-16.67478$

$-16.81031$

$-16.56257$

$-18.39725$

$-18.29829$

$-17.98651$

$-18.07596$

$-18.19924$

$-17.58686$

$-17.91869$

$-17.50549$

$-17.44108$

$-17.79281$

$-17.56302$

$-17.05968$

$-18.10818$

$-18.46033$

$-19.24289$

$-19.58091$

$-18.85095$

$-19.60441$

$-19.47361$

\subsection{7}

0.55578

0.54823

0.53936

0.55545

0.53758

0.59998

0.57896

0.54698

0.57635

0.64250

0.76418

0.53934

0.52778

0.91869

0.66330

0.54887

0.87827

0.61839

0.67100

0.73070

0.53585

0.61177

0.56749

0.70811

0.76294

0.76060

0.71295

0.99993

0.56157

0.73487

0.56463

0.54410

1.14251

1.29018

0.90767

0.71587

I. 06887

0.88441

0.96944

I. 52425

l. 10832

1. 28553
Surface Contamination

avg sd s

$-12.54047$

$-12.41882$

$-12.15366$

$-11.99020$

$-12.12509$

$-12.27281$

$-12.38737$

$-12.21055$

$-11.81370$

$-11.45514$

$-11.00443$

$-10.27719$

$-10.73012$

$-10.84199$

$-11.83045$

$-12.25905$

$-10.48185$

$-11.82445$

$-10.75797$

$-11.20361$

$-11.70467$

$-10.69890$

0.59688

0.61017

0.57932

0.57874

0.59978

0.58404

0.66868

0.62884

0.60243

0.63204

0.63017

0.77301

0.55554

0.54226

0.93321

0.75083

0.56841

$0.93 \mathrm{~B} 8]$

0.60525

0.70757

0.79134

$-10.85985$

$-10.57579$

0.55872

0.64245

$-12.57493$

$-12.47523$

$-12.20396$

$-12.23281$

$-12.47248$

$-11.75843$

$-12.10114$

$-11.71040$

$-11.63445$

$-11.99342$

$-11.79612$

$-11.13897$

$-12.25915$

0.55372 
IABLE 13. (contd)

Time-Integrated

Census Air Concentration$$
\text { D }
$$

KL1 -11.96831

$\begin{array}{ll}K L 2 & -12.30322\end{array}$

KL3 -12.11740

KL4 -12.96273

WA1 $\quad-9.52856$

WA2 -9.02253

HA3 -8.71977

WA4 -9.04772

WA5 -9.27038

WA6 -9.46622

WA7 -9.58808

WA8 -9.43028

HA9 -9.31798

WA10 -9.38734

YAl -10.44023

YA2 - 11.39583

YA5 -11.23484

YA6 -10.11296

YA7 -11.03398

YA9 $\quad-9.76213$

YA10 -10.65093

YA11 -11.32256

M01 $\quad-10.69056$

M02 -10.06992

MD3 -10.92376

UM1 $\quad-9.23074$

UM2 $\quad-9.40004$

UM3 $\quad-9.36154$

UM4 $\quad-8.93264$

UM5 $\quad-9.39750$

UM6 $\quad-9.11888$

UM7 $\quad-9.69721$

UM8 $\quad-9.28767$

UM9 $\quad-9.62267$

UM10 $\quad-9.55405$

UMI $1 \quad-9.33289$

UM12 -9.35880

UM13 -9.54884

UM14 $\quad-9.54348$ sd

1.73244

1.87140

0.54432

2.02394

0.63706

0.56522

0.55229

0.56020

0.54900

0.55715

0.55986

0.55310

0.55036

0.55455

1.76717

2.09708

1.41925

1.43942

1.69900

1.06905

1.21811

1. 59321

1. 30464

0.60436

1.40216

0.55106

0.54569

0.60068

0.53820

0.53888

0.54995

0.63637

0.54998

0.59083

0.59152

0.54623

0.60579

0.54834

0.57265
Deposition Rate

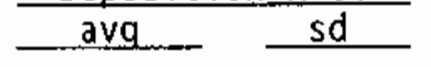

$-19.89011$

$-20.08207$

$-20.53104$

$-20.30619$

$-17.94220$

$-17.43616$

$-17.13341$

$-17.46136$

$-17.68402$

$-17.87986$

$-18.00172$

$-17.84391$

$-17.73161$

$-17.80097$

$-18.51429$

$-19.3524 \mathrm{~g}$

$-19.64847$

$-18.39446$

$-19.04016$

$-18.17577$

$-19.06456$

$-19.50617$

$-18.99831$

$-18.48356$

$-19.23963$

$-17.64438$

$-17.81368$

$-17.77518$

$-17.34628$

$-17.81114$

$-17.53251$

$-18.11085$

$-17.70130$

$-18.03631$

$-17.96769$

$-17.74653$

$-17.77244$

$-17.96247$

$-17.95712$
1.15403

1.18510

0.54429

0.84734

0.63703

0.56506

0.55225

0.56010

0.54890

0.55704

0.55983

0.55314

0.55040

0.55454

1.16262

1.51324

1.41924

1.19229

0.99694

1.06902

1.21813

1.29941

1.13903

0.60439

1. 20943

0.55104

0.54558

0.60062

0.53812

0.53880

0.54995

0.63648

0.55004

0.59073

0.59155

0.54614

0.60578

0.54823

0.57263
Surface Contamination

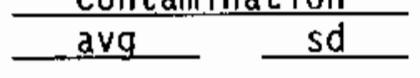

$-14.45810$

1.80716

$-14.51001$

$-14.59744$

$-15.31263$

$-11.98649$

$-11.50600$

$-11.20484$

$-11.55516$

$-11.78973$

$-11.99115$

$-12.12787$

$-11.96037$

$-11.82981$

$-11.91733$

$-12.75934$

$-13.87131$

$-13.70025$

$-12.57224$

$-13.50551$

$-12.16432$

$-13.11556$

$-13.94636$

$-13.09853$

$-12.34475$

$-13.26487$

$-11.74228$

$-11.91710$

$-11.68925$

$-11.34205$

$-11.90763$

$-11.54044$

$-12.00723$

$-11.77879$

$-11.93189$

$-12.02174$

$-11.84210$

$-11.69726$

$-12.09151$

$-12.07110$
1.65477

0.54061

1.99338

0.72637

0.57152

0.55360

0.56961

0.54394

0.56316

0.55494

0.57325

0.55809

1.56045

1.96377

1.32020

1.45777

1.52129

1.10039

1.31963

1.68388

1.33551

0.58646

1.33779

0.56021

0.56874

0.61127

0.55351

0.56325

0.58030

0.62810

0.57075

0.58430

0.61709

0.56665

0.61388

0.57751

0.61662
0.56450 
TABLE 14. Computational Results for December. All numerical values are logarithms to base 10. Units are as follows: time-integrated air concentration, $\left[(\mathrm{Ci}-\mathrm{s}) / \mathrm{m}_{2}^{3}\right] / \mathrm{Ci}$; deposition rate, $\left[\left(\mathrm{Ci} / \mathrm{m}^{2}\right) / \mathrm{s}\right] / \mathrm{C}$; surface contamination, $\left(\mathrm{C} i / \mathrm{m}^{2}\right) / \mathrm{C} i$.

Time-Integrated

Census Air Concentration

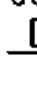$$
\text { Div }
$$

\begin{tabular}{|c|c|c|c|}
\hline & & & \\
\hline $\mathrm{ADI}$ & -10.94975 & 1.60617 & -19.18663 \\
\hline $\mathrm{AD2}$ & -10.42204 & 0.87062 & -18.77768 \\
\hline $\mathrm{AD3}$ & -10.00899 & 0.69038 & -18.43687 \\
\hline AD4 & -9.88581 & 0.61147 & -18.31369 \\
\hline AD5 & -10.24098 & 1.17348 & -18.57762 \\
\hline AD6 & -10.79442 & 1.91105 & -18.95373 \\
\hline AD7 & -10.54541 & 1.17636 & -18.97328 \\
\hline ADB & -10.19505 & 0.95434 & -18.62292 \\
\hline AD9 & -9.77363 & 0.89069 & -18.20150 \\
\hline AD10 & -9.63503 & 1.32499 & -17.96922 \\
\hline$B E 1$ & -8.34562 & 0.64391 & -16.77350 \\
\hline BE2 & -7.86395 & 0.98180 & -16.29182 \\
\hline$B E 3$ & -8.05729 & 0.53664 & -16.48517 \\
\hline BE4 & -8.24528 & 0.54490 & -16.67316 \\
\hline BE5 & -9.50805 & 1.15091 & -17.93592 \\
\hline BE6 & -10.26380 & 1.23594 & -18.69168 \\
\hline BE7 & -7.88001 & 0.54696 & -16.30788 \\
\hline BE8 & -9.47573 & 1.14982 & -17.90360 \\
\hline BE9 & -8.17113 & 0.59026 & -16.59901 \\
\hline FRI & -9.06565 & 0.90716 & -17.49353 \\
\hline FR2 & -9.61473 & 0.98324 & -18.04261 \\
\hline FR3 & -8.10958 & 0.55681 & -16.53745 \\
\hline FR4 & -8.79071 & 0.79064 & -17.21859 \\
\hline FR5 & -8.18267 & 0.60491 & -16.61055 \\
\hline GR2 & -10.76077 & 0.81412 & -19.18864 \\
\hline GR3 & -10.43346 & 0.79687 & -18.86134 \\
\hline GR4 & -10.15602 & 0.74407 & -18.58390 \\
\hline GR5 & -10.13712 & 0.83313 & -18.56499 \\
\hline GR6 & -11.34958 & 1.89037 & -19.33659 \\
\hline GR7 & -9.76238 & 0.64202 & -18.19026 \\
\hline GR8 & -10.12844 & 0.77662 & -18.55632 \\
\hline GR9 & -9.59648 & 0.67307 & -18.02436 \\
\hline GR10 & -9.47664 & 0.64462 & -17.90451 \\
\hline GR11 & -10.13667 & I. 52385 & -18.39609 \\
\hline GR12 & -9.64141 & 1.36039 & -18.06928 \\
\hline GR1 & -9.08351 & 1.48389 & -17.51138 \\
\hline GR14 & -10.23286 & 0.84312 & -18.66074 \\
\hline KII & -11.80893 & 2.04404 & -19.79605 \\
\hline $\mathrm{KI} 2$ & -12.59253 & 2.00530 & -20.29635 \\
\hline KI7 & -12.32944 & 1.12946 & -20.75732 \\
\hline KI8 & -10.75228 & 1.96028 & -18.82170 \\
\hline KI9 & -12.18225 & 1.71515 & -20.10177 \\
\hline KIIO & -11.10435 & 0.91306 & -19.53223 \\
\hline
\end{tabular}

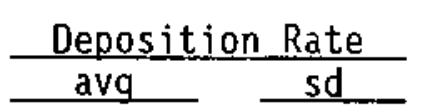

1.26810

0.62811

0.69031

0.61144

0.96969

1.44078

1.17638

0.95435

0.89069

1.13660

0.64387

0.98181

0.53661

0.54485

1.15092

1.23596

0.54689

1.14975

0.59031

0.90712

0.98322

0.55677

0.79058

0.60482

0.81409

0.79679

0.74399

0.83319

1. 32307

0.64200

0.77658

0.67305

0.64461

1.20274

1.36041

1. 48391

0.84307

1.65127

1.73366

1. 12948

1.45947

1.12033

0.91300

\begin{tabular}{lc}
\multicolumn{2}{c}{ Surface } \\
\cline { 1 - 1 } Contamination \\
\cline { 1 - 1 } avg & sd \\
\hline-13.44934 & 1.74718 \\
-12.91444 & 0.89493 \\
-12.48096 & 0.60229 \\
-12.37317 & 0.59675 \\
-12.72068 & 1.26634 \\
-13.03583 & 1.62017 \\
-13.04888 & 1.36422 \\
-12.67903 & 1.11458 \\
-12.15270 & 0.87036 \\
-12.08071 & 1.31705 \\
-10.86543 & 0.67495 \\
-10.35891 & 1.03247 \\
-10.66173 & 0.60554 \\
-10.84047 & 0.60859 \\
-11.98547 & 1.21226 \\
-12.75802 & 1.27318 \\
-10.46528 & 0.60325 \\
-12.02765 & 1.15840 \\
-10.71573 & 0.63069 \\
-11.54712 & 0.98425 \\
-12.14054 & 1.00942 \\
-10.69426 & 0.61602 \\
-11.35704 & 0.88265 \\
-10.75716 & 0.63450 \\
-13.26342 & 0.69998 \\
-12.91582 & 0.69484 \\
-12.58097 & 0.68914 \\
-12.69825 & 0.66706 \\
-13.91895 & 1.78578 \\
-12.24181 & 0.64428 \\
-12.57357 & 0.70200 \\
-12.08023 & 0.66189 \\
-11.97770 & 0.62228 \\
-12.69673 & 1.48246 \\
-12.27198 & 1.49278 \\
-11.63981 & 1.61853 \\
-12.78175 & 0.67026 \\
-14.24951 & 2.01516 \\
-14.96366 & 1.98166 \\
-14.66412 & 1.10570 \\
-13.27795 & 0.90263
\end{tabular}


TABLE 14. (contd)

Time-Integrated

Census Air Concentration

\begin{tabular}{llccc} 
Div. & avg & sd & & avg \\
\cline { 3 - 4 } KL1 & -13.10562 & 1.85393 & -20.96770 \\
KL2 & -13.19868 & 1.95279 & -20.95570 \\
KL3 & -14.99228 & 0.87848 & -22.73836 \\
KL4 & -13.95980 & 0.61525 & -22.38767 \\
WA1 & -10.35587 & 0.94297 & -18.78375 \\
WA2 & -9.28589 & 0.71369 & -17.71376 \\
WA3 & -8.71377 & 0.60299 & -17.14164 \\
WA4 & -9.09064 & 0.64566 & -17.51852 \\
WA5 & -9.77276 & 0.76295 & -18.20063 \\
WA6 & -10.42799 & 1.51271 & -18.52770 \\
WA7 & -10.77492 & 1.63766 & -18.84985 \\
WA8 & -9.79354 & 0.73135 & -18.22142 \\
WA9 & -9.29167 & 0.59725 & -17.71954 \\
WA10 & -9.68993 & 0.64246 & -18.11780 \\
YA1 & -10.77687 & 2.05503 & -18.85818 \\
YA2 & -10.64222 & 0.84636 & -19.07010 \\
YA5 & -11.06357 & 1.05794 & -19.49145 \\
YA6 & -10.85782 & 2.34797 & -18.60198 \\
YA7 & -12.43583 & 1.98833 & -20.10939 \\
YA9 & -10.18466 & 1.51272 & -18.49583 \\
YA10 & -11.40220 & 1.86630 & -19.54244 \\
YA11 & -13.17265 & 2.05544 & -20.75872 \\
M01 & -10.59721 & 1.14633 & -19.02509 \\
M02 & -10.01409 & 0.60525 & -18.44197 \\
M03 & -10.61719 & 1.00788 & -19.04507 \\
UM1 & -9.11142 & 0.59936 & -17.53930 \\
UM2 & -9.43911 & 0.61719 & -17.86699 \\
UM3 & -9.26019 & 0.64826 & -17.68806 \\
UM4 & -8.74174 & 0.55141 & -17.16961 \\
UM5 & -9.35720 & 0.62126 & -17.78508 \\
UM6 & -8.93028 & 0.57466 & -17.35815 \\
UM7 & -9.64267 & 0.65975 & -18.07055 \\
UM8 & -9.17707 & 0.58962 & -17.60494 \\
UM9 & -9.54823 & 0.63507 & -17.97611 \\
UM10 & -9.48721 & 0.60679 & -17.91508 \\
UM11 & -9.28078 & 0.60034 & -17.70865 \\
UM12 & -9.23083 & 0.64302 & -17.65871 \\
UM13 & -9.63351 & 0.66273 & -18.06138 \\
UM14 & -9.50865 & 0.67761 & -17.93652
\end{tabular}

Surface Contamination

avg

1.46435

1.63872

0.56460

0.61520

0.94292

0.71374

0.60305

0.64569

0.76296

0.70258

0.98035

0.73137

0.59723

0.64252

1.62769

0.84638

1.05792

1.53900

1.30950

1.30707

1.48977

1.53434

1.14639

0.60530

1.00785

0.59939

0.61723

0.64829

0.55140

0.62129

0.57465

0.65977

0.58967

0.63507

0.60678

0.60036

0.64309

0.66274

0.67766
$-15.34562$

$-15.43134$

$-17.19097$

$-16.02952$

$-12.91075$

$-11.93759$

$-11.30337$

$-11.71398$

$-12.41977$

$-12.87048$

$-13.23108$

$-12.42094$

$-11.93627$

$-12.31279$

$-12.93999$

$-12.74576$

$-13.14295$

$-13.01150$

$-14.36648$

$-12.53594$

$-13.65835$

$-15.20161$

$-13.16250$

$-12.66888$

$-13.24300$

$-11.70136$

$-12.08515$

$-11.89908$

$-11.33017$

$-12.00683$

$-11.54250$

$-12.30166$

$-11.74259$

$-12.22049$

$-12.04908$

$-11.92402$

$-11.85033$

$-12.30421$

$-12.18829$
1.85989

1.87893

0.91219

0.61229

1.04690

0.77943

0.65692

0.70302

0.84659

1.18070

1. 44779

0.81885

0.64616

0.71933

1.98490

0.88215

1.09356

2. 31614

1.80039

1.65806

1.73231

1.99937

1. 25442

0.73339

1.15277

0.66122

0.66885

0.75048

0.61480

0.66587

0.63656

0.77521

0.64315

0.76272

0.65903

0.64778

0.73078

0.72359

0.70645 



\section{REFERENCES}

Ballinger, M. Y., and R. A. Hall. 1991. A History of Major Hanford Operations Involving Radioactive Material. PNL-6964 HEDR, Pacific Northwest Laboratory, Richland, Washington.

Briggs, G. A. 1984. "Plume Rise and Buoyancy Effects." In Atmospheric Science and Power Production, ed. D. Randerson, Pp. 327-366. D0E/TIC-27601, U.S. Department of Energy, Washington, D.C.

Pacific Northwest Laboratory (PNL). 1991. Air Pathway Report, Phase I of the Hanford Environmental Dose Reconstruction Project. PNL-7412 HEDR, Pacific Northwest Laboratory, Richland, Washington.

Ramsde11, J. V., G. F. Athey, and C. S. Glantz. 1983. MESOI Version 2.0: An Interactive Puff Dispersion Model With Deposition and Decay. NUREG/CR-3344, U.S. Nuclear Regulatory Commission, Washington, D.C.

Ramsdell, J. V., G. F. Athey, T. J. Bander, and R. I. Scherpelz. 1988. The MESORAD Dose Assessment Code, Volume 2: Computer Code. NUREG/CR-4000 v.2, U.S. Nuclear Regulatory Commission, Washington, D.C.

Ramsde11, J. V. 1991. Atmospheric Transport and Dispersion Modeling for the Hanford Environmental Dose Reconstruction Project. PNL-7198 HEDR, Pacific Northwest Laboratory, Richland, Washington.

Ramsdell, J. V., and K.W. Burk. 1991. MESOILT2, a Lagrangian Trajectory Climatological Dispersion Model. PNL-7340 HEDR, Pacific Northwest Laboratory, Richland, Washington.

Scherpelz, R. I., T. J. Bander, G. F. Athey, and J. V. Ramsdell. 1986. The MESORAD Dose Assessment Code, Volume 1: Technical Basis. NUREG/CR-4000 v.1, U.S. Nuclear Regulatory Commission, Washington, D.C.

Smith, J. M. 1950. Hanford Works Stacks - Construction and Usage. HW-1B143 (declassified), Hanford Atomic Products Operation, General Electric Company, Richland, Washington. 

APPENDIX

SUMMARY OF TSP COMMENTS AND BATTELLE RESPONSES 


\section{SUMMARY OF TSP COMMENTS AND BATTELLE RESPONSES}

Document Number PNL-7199 HEDR

\begin{tabular}{|c|c|c|c|c|}
\hline $\begin{array}{l}\text { Comment } \\
\text { Number }\end{array}$ & Conimenter & $\begin{array}{l}\text { Page, } \\
\text { Paragraph }\end{array}$ & Comment Summary & Resolution \\
\hline 1. & $\begin{array}{l}\text { Vietchau } \\
\text { Nguyen }\end{array}$ & & $\begin{array}{l}\text { Monthly time-integrated concentrations are used } \\
\text { in the MESOILT2 model, thus ignoring the irrever- } \\
\text { sible health impact peak concentrations may cause } \\
\text { when high releases of iodine-13l occur in the } \\
\text { shorter time scale. }\end{array}$ & $\begin{array}{l}\text { MESOILT2 can use release rates } \\
\text { defined hourly and makes its time- } \\
\text { integrated air concentration and } \\
\text { deposition calculations using time } \\
\text { steps that are no longer than } 15 \\
\text { minutes. }\end{array}$ \\
\hline 2. & VN & Page 3 & $\begin{array}{l}\text { A clarification on the current status and } \\
\text { upgraded capabil ities of MESOILT2 must be } \\
\text { provided. Model deficiencies and methods of } \\
\text { resolution must also be detailed as well as model } \\
\text { limitations, especially with regard to the effect } \\
\text { of topography. }\end{array}$ & $\begin{array}{l}\text { The MESOILT2 mode] is fully } \\
\text { described in PNL-7340 HEDR. } \\
\text { Extensive modifications are being } \\
\text { made to the model and will be doc- } \\
\text { umented when complete. }\end{array}$ \\
\hline 3. & VN & Page 5 & $\begin{array}{l}\text { Wind fields are computed using interpolation and } \\
\text { adjusted for terrain effects. This approach } \\
\text { raises concerns on the additional uncertainty } \\
\text { associated with the calculated values caused by } \\
\text { interpolation. }\end{array}$ & $\begin{array}{l}\text { Alternative methods for estimating } \\
\text { wind fields are being evaluated. }\end{array}$ \\
\hline 4. & VN & & $\begin{array}{l}\text { Sample runs (including input data and actual out- } \\
\text { put files) are not provided as requested for ver- } \\
\text { ification. }\end{array}$ & $\begin{array}{l}\text { This is not the appropriate place } \\
\text { for the requested information. } \\
\text { The information was provided to } \\
\text { Or. Nguyen in July } 1990 \text {. }\end{array}$ \\
\hline 5. & VN & $\begin{array}{l}\text { Tables } 3 \text { - } \\
14\end{array}$ & $\begin{array}{l}\text { Sensitivity of computational results has not been } \\
\text { addressed. This will help narrow down the com- } \\
\text { putational variance of the dose estimates. }\end{array}$ & $\begin{array}{l}\text { A sensitivity analysis is beyond } \\
\text { the scope of this report. An } \\
\text { evaluation of sensitivity and } \\
\text { uncertainty of the model will be } \\
\text { made following completion of the } \\
\text { model revisions. }\end{array}$ \\
\hline
\end{tabular}




\begin{tabular}{|c|c|c|c|c|}
\hline $\begin{array}{l}\text { Comment } \\
\text { Number }\end{array}$ & Commenter & $\begin{array}{c}\text { Page, } \\
\text { Paragraph }\end{array}$ & Comment Summary & Resolution \\
\hline 6. & $\begin{array}{l}\text { R.L. } \\
\text { Morrill }\end{array}$ & & $\begin{array}{l}\text { This report is too brief and skeletal. I want } \\
\text { more meat. }\end{array}$ & $\begin{array}{l}\text { The scope of this report is } 1 \text { im- } \\
\text { ited to documentation of the input } \\
\text { to the atmospheric transport model } \\
\text { used in Phase I and of the output } \\
\text { from the atmospheric model that } \\
\text { was passed to the dose calculation } \\
\text { task. It does not include an } \\
\text { analysis of the atmospheric model } \\
\text { output. The restructured model } \\
\text { will be fully evaluated and } \\
\text { described. }\end{array}$ \\
\hline 7. & RLM & & $\begin{array}{l}\text { A map showing the grid system of the rectangular } \\
\text { domain overlying the study area is needed. }\end{array}$ & $\begin{array}{l}\text { Figures } 1 \text { and } 2 \text { show the relation- } \\
\text { ship between the atmospheric model } \\
\text { domain and the } 10 \text {-county study } \\
\text { area. The lower left corner of } \\
\text { the model domain corresponds to } \\
\text { grid location } 1,1 \text {, and the upper } \\
\text { right corner corresponds to loca- } \\
\text { tion } 31,31 \text {. Addition of the grid } \\
\text { lines would clutter the figures } \\
\text { without adding a significant } \\
\text { amount of information. }\end{array}$ \\
\hline 8. & RLM & & $\begin{array}{l}\text { There should be several maps, as every other } \\
\text { month, converting the data in the tables to con- } \\
\text { tour isolines, say of air concentrations or sur- } \\
\text { face contamination. }\end{array}$ & $\begin{array}{l}\text { The requested maps are outside the } \\
\text { scope of this report. Such maps } \\
\text { will be available from analysis of } \\
\text { the restructured model. }\end{array}$ \\
\hline
\end{tabular}


SUMMARY OF TSP COMMENTS AND BATTELLE RESPONSES

Document Number PNL-7199 HEDR

\begin{tabular}{|c|c|c|c|c|}
\hline $\begin{array}{l}\text { Comment } \\
\text { Number }\end{array}$ & Commenter & $\begin{array}{l}\text { Page, } \\
\text { Paragraph }\end{array}$ & Comment Summary & Resolution \\
\hline 9. & RLM & & $\begin{array}{l}\text { Do these model results make use of the six typi- } \\
\text { cal wind patterns or not? }\end{array}$ & $\begin{array}{l}\text { The Phase I results are based on } \\
\text { model calculations using actual } \\
\text { wind data to generate the wind } \\
\text { fields. Using patterns was not } \\
\text { necessary. }\end{array}$ \\
\hline 10 & RLM & Pages 3,5 & $\begin{array}{l}\text { Could high precipitation away from Hanford lead } \\
\text { to elevated concentrations and surface con- } \\
\text { tamination? If so, then the results here would } \\
\text { not capture that. }\end{array}$ & $\begin{array}{l}\text { Precipitation results in higher } \\
\text { deposition rates than does dry } \\
\text { deposition. However, the fraction } \\
\text { of time that precipitation occurs } \\
\text { at any location in the mode] } \\
\text { domain is small. Therefore, the } \\
\text { expected effect of not including } \\
\text { precipitation in the calculations } \\
\text { is small. This expectation was } \\
\text { confirmed in a subsequent set of } \\
\text { calculations. Precipitation data } \\
\text { will be included in final dose } \\
\text { estimates. }\end{array}$ \\
\hline 11. & RLM & & $\begin{array}{l}\text { What I'd like is a better sense of where the num- } \\
\text { bers come from. Shouldn't we be talking about a } \\
\text { distribution of hours of different wind dir- } \\
\text { ections and speeds, instead of crude monthly } \\
\text { averages? }\end{array}$ & $\begin{array}{l}\text { The Phase I model calculations } \\
\text { were made using observed meteor- } \\
\text { ological data, not with averages } \\
\text { or summarized data. Results of } \\
\text { future models can be presented in } \\
\text { time-location representations. }\end{array}$ \\
\hline 12 . & RLM & & $\begin{array}{l}\text { This atmospheric modeling component is extremely } \\
\text { important. Its meaning and operation must be } \\
\text { made clearer to us and ultimately to the public. }\end{array}$ & $\begin{array}{l}\text { PNL }-7340 \text { HEDR provides a complete } \\
\text { description of the atmospheric } \\
\text { model used in the Phase I } \\
\text { calculations. }\end{array}$ \\
\hline
\end{tabular}


SUMMARY OF TSP COMMENTS AND BATTELLE RESPONSES

Document Number PNL-7199 HEDR

Document Title Atmospheric Transport Modeling and Input Data for Phase I of the HEDR Project

\begin{tabular}{|c|c|c|c|c|}
\hline $\begin{array}{l}\text { Comment } \\
\text { Number }\end{array}$ & Commenter & $\begin{array}{c}\text { Page, } \\
\text { Paragraph }\end{array}$ & Comment Summary & Resolution \\
\hline 13. & R. Cook & & $\begin{array}{l}\text { The document should plot data (for example, in } \\
\text { Table } 3 \text { ) on large-scale maps, with contour lines } \\
\text { connecting the points with equivalent values. }\end{array}$ & $\begin{array}{l}\text { The requested plots are outside } \\
\text { the scope of this report. This } \\
\text { information will be available for } \\
\text { final meteorological representa- } \\
\text { tions. }\end{array}$ \\
\hline i 4 . & $\mathrm{RC}$ & & $\begin{array}{l}\text { Discuss the basis for the deposition velocity for } \\
\text { iodine and other isotopes, and identify the val- } \\
\text { idity of the respective values. Identify uncer- } \\
\text { tainties in the values. Specify best estimate, l } \\
\text { sigma and } 2 \text { sigma values. }\end{array}$ & $\begin{array}{l}\text { MESOILT2 uses a constant deposi- } \\
\text { tion velocity of } 1 \text { cm/sec. Use of } \\
\text { this value is consistent with } \\
\text { practice in many models used by } \\
\text { the NRC and EPA. A more refined } \\
\text { method of treating dry deposition } \\
\text { is being added to the model as the } \\
\text { model is being revised. }\end{array}$ \\
\hline 15. & $R C$ & & $\begin{array}{l}\text { To correlate wind pattern data with iodine-131 } \\
\text { levels around Hanford, provide contour plots of } \\
\text { the iodine-131 data as a function of time--by the } \\
\text { month. }\end{array}$ & $\begin{array}{l}\text { The requested plots are outside } \\
\text { the scope of the report. See } \\
\text { response to comment } 13 \text {. }\end{array}$ \\
\hline 16. & D.S. Barth & $\begin{array}{l}\text { Page } 2 \\
\text { para } 5\end{array}$ & $\begin{array}{l}\text { What are the criteria on the basis of which the } \\
\text { test results were found to be satisfactory? }\end{array}$ & $\begin{array}{l}\text { Text was rewritten to state extent } \\
\text { and results of the tests. }\end{array}$ \\
\hline 17 . & DSB & $\begin{array}{l}\text { Page } 6 \\
\text { para } 6\end{array}$ & $\begin{array}{l}\text { Why is it not possible to spot check the actual } \\
\text { data against the } 1983-87 \text { data for similarities or } \\
\text { differences for at least a few of the most impor- } \\
\text { tant months? }\end{array}$ & $\begin{array}{l}\text { The model has been run with the } \\
\text { actual meteorological data; see } \\
\text { Figure } 2.7 \text { of the Air Pathway } \\
\text { Report (PNL-7412 HEDR). }\end{array}$ \\
\hline 18. & DSB & $\begin{array}{l}\text { Page } 10 \\
1 \text { ines } 10- \\
11\end{array}$ & $\begin{array}{l}\text { Why was not a flow of } 45,000 \mathrm{ft}^{3} / \mathrm{min} \text { assumed for } \\
\text { the imaginary stack replacing the two real } \\
\text { stacks? }\end{array}$ & $\begin{array}{l}\text { The flow was used to estimate the } \\
\text { initial dilution of the iodine- } \\
\text { l3l. Assuming a flow equal to } \\
\text { twice the flow of a single stack }\end{array}$ \\
\hline
\end{tabular}




\section{SUMMARY OF TSP COMMENTS AND BATTELLE RESPONSES}

Document Number PNL-7199 HEDR

Document Title Atmospheric Transport Modeling and Input Data for Phase I of the HEOR Project

\begin{tabular}{|c|c|c|c|c|}
\hline $\begin{array}{l}\text { Comment } \\
\text { Number }\end{array}$ & Commenter & $\begin{array}{c}\text { Page, } \\
\text { Paragraph }\end{array}$ & Comment Summary & Resolution \\
\hline & & & & $\begin{array}{l}\text { would reduce the initial concen- } \\
\text { tration by a factor of } 2 \text {. In } \\
\text { general, however, the concentra- } \\
\text { tions and therefore the doses at } \\
\text { locations off the Hanford Site are } \\
\text { not affected by the assumed flow } \\
\text { because the volume of the puffs is } \\
\text { large compared with the initial } \\
\text { volume. }\end{array}$ \\
\hline 19. & DSB & & $\begin{array}{l}\text { It would be a useful exercise to calculate, for a } \\
\text { few selected months, biweekly or weekly averages } \\
\text { and variances so that these could be compared to } \\
\text { the monthly average results. }\end{array}$ & $\begin{array}{l}\text { The revised atmospheric model will } \\
\text { generate daily values of the time- } \\
\text { integrated air concentrations and } \\
\text { deposition for use in dose } \\
\text { calculations. }\end{array}$ \\
\hline 20 . & $\begin{array}{l}\text { Giyn G. } \\
\text { Caldwel] }\end{array}$ & & $x^{2}$ & $\begin{array}{l}\text { The model can be extended to } \\
\text { handle as many radionuclides as } \\
\text { needed for the dose calculation. } \\
\text { The number of model runs will } \\
\text { depend more on the number and var- } \\
\text { iety of sources and their charac- } \\
\text { teristics than on the number of } \\
\text { radionuclides. MESOILT2 can treat } \\
4 \text { sources simultaneously. This } \\
\text { number can also be expanded if } \\
\text { necessary. }\end{array}$ \\
\hline 21. & GGC & & $\begin{array}{l}\text { Were any radionuclides handled as particulates; } \\
\text { did particulates exist? }\end{array}$ & $\begin{array}{l}\text { The Phase I study dealt only with } \\
\text { iodine-131. It was treated as a } \\
\text { depositing species without further } \\
\text { characterization. The deposition }\end{array}$ \\
\hline
\end{tabular}


SUMMARY OF TSP COMMENTS AND BATTELLE RESPON'SES

Document Number PNL-7199 HEDR

Document Title Atmospheric Transport Modeling and Input Data for Phase I of the HEDR Project

\begin{tabular}{|c|c|c|c|c|}
\hline $\begin{array}{l}\text { Comment } \\
\text { Number }\end{array}$ & Commenter & $\begin{array}{c}\text { Page, } \\
\text { Paragraph }\end{array}$ & Comment Summary & Resolution \\
\hline 22. & $\mathrm{GGC}$ & & $\begin{array}{l}\text { How were the deposition velocities used } \\
\text { determined; from what data? }\end{array}$ & $\begin{array}{l}\text { velocity assumed for iodine-l3l is } \\
\text { characteristic of reactive gases. } \\
\text { The revised atmospheric model wilt } \\
\text { calculate deposition velocities } \\
\text { for both reactive gases and par- } \\
\text { ticulates based on atmospheric } \\
\text { conditions and surface roughness. } \\
\text { The } 0.01-\mathrm{m} / \mathrm{sec} \text { deposition velocity } \\
\text { assumed in the Phase I calcula- } \\
\text { tions is consistent with NRC and } \\
\text { EPA regulatory practice. This } \\
\text { velocity is near the high end of } \\
\text { the range of experimental values } \\
\text { reported in Sehmel's l980 review } \\
\text { article (Atmospheric Environment } \\
14: 983-1011 \text {. }\end{array}$ \\
\hline 23. & $\mathrm{GGC}$ & $\begin{array}{l}\text { Page } 4, \\
\text { para } 1 \\
\text { 1ines } 1-2\end{array}$ & $\begin{array}{l}\text { Are node distances acceptable, were others } \\
\text { evaluated; if not, why not; if so, what led to } \\
\text { use of } 5 \text {-mile spacing? }\end{array}$ & $\begin{array}{l}\text { The 5-mile node spacing was deter- } \\
\text { mined from overall domain size and } \\
\text { the existing model structure. No } \\
\text { other spacing was considered, } \\
\text { because the spacing was not a } \\
\text { critical factor in assessing } \\
\text { whether the objectives of Phase I } \\
\text { were met. The results of Phase I } \\
\text { indicate that } 5 \text {-mile node spacing } \\
\text { is satisfactory for making offsite } \\
\text { dose estimates. }\end{array}$ \\
\hline
\end{tabular}


SUMMARY OF TSP COMMENTS AND BATTELLE RESPONSES

Document Number PNL-719g HEDR

Document Title Atmospheric Transport Modeling and Input Data for Phase I of the HEDR Project

\begin{tabular}{|c|c|c|c|c|}
\hline $\begin{array}{l}\text { Comment } \\
\text { Number }\end{array}$ & Commenter & $\begin{array}{l}\text { Page, } \\
\text { Paragraph }\end{array}$ & Comment Summary & Resolution \\
\hline 24. & GGC & Page 4 & $\begin{array}{l}\text { How will high terrain be managed in later } \\
\text { versions and the final model? }\end{array}$ & $\begin{array}{l}\text { A method for treating terrain in } \\
\text { the revised model has not been } \\
\text { established. }\end{array}$ \\
\hline 25. & GGC & Page 6 & $\begin{array}{l}\text { Is assumed uniformity of the atmospheric } \\
\text { stability supportable by existing data? }\end{array}$ & $\begin{array}{l}\text { The assumption is probably accept- } \\
\text { able in general but is certainly } \\
\text { incorrect in specific cases. } \\
\text { Atmospheric stability will be a } \\
\text { function of location in the } \\
\text { revised model. }\end{array}$ \\
\hline 26. & GGC & Page 6 & When will precipitation be included? & $\begin{array}{l}\text { MESOILT2 is capable of treating } \\
\text { precipitation. Precipitation data } \\
\text { were not used in the Phase I cal- } \\
\text { culations but have been used in } \\
\text { subsequent calculations without } \\
\text { changing the general character of } \\
\text { the results. }\end{array}$ \\
\hline 27. & GGC & $\begin{array}{l}\text { Page } 10 \\
\text { para } 2\end{array}$ & Will final model use both stacks and actual data? & $\begin{array}{l}\text { Yes, future model calculations } \\
\text { will make use of all avajlable } \\
\text { data on release locations and } \\
\text { release rates. }\end{array}$ \\
\hline 28. & GGC & Page 13 & $\begin{array}{l}\text { This statement about decay seems to contradict } \\
\text { that made earlier in the text. }\end{array}$ & $\begin{array}{l}\text { Both statements were deleted; the } \\
\text { text has been revised for clarity. }\end{array}$ \\
\hline
\end{tabular}




\section{SUMMARY OF TSP COMMENTS AND BATTELLE RESPONSES}

Document Number PNL-7199 HEDR

\begin{tabular}{|c|c|c|c|c|}
\hline $\begin{array}{l}\text { Comment } \\
\text { Number }\end{array}$ & Commenter & $\begin{array}{l}\text { Page, } \\
\text { Paragraph }\end{array}$ & Comment Summary & Reso'Tution \\
\hline 29. & $\mathrm{GGC}$ & $\begin{array}{l}\text { Page } 20, \\
\text { para } 1\end{array}$ & $\begin{array}{l}\text { On what data were these assumed background con- } \\
\text { centrations and rates based? }\end{array}$ & $\begin{array}{l}\text { The text has been rewritten to } \\
\text { better describe the numbers. The } \\
\text { numbers were used as minimum val- } \\
\text { ues to avoid problems associated } \\
\text { with using logarithms of very } \\
\text { small numbers in computing the } \\
\text { averages and standard deviations } \\
\text { of values for the census } \\
\text { divisions. }\end{array}$ \\
\hline 30. & GGC & $\begin{array}{l}\text { Tables } 3- \\
14\end{array}$ & $\begin{array}{l}\text { Could data be plotted on a map such as Figure } 3 \text {; } \\
\text { would such a map be more understandable to the } \\
\text { public? }\end{array}$ & $\begin{array}{l}\text { The requested figures are outside } \\
\text { the scope of the report. Such } \\
\text { maps could be developed from } \\
\text { restructured model data. }\end{array}$ \\
\hline 31. & $\begin{array}{l}\text { David } \\
\text { Price }\end{array}$ & & $\begin{array}{l}\text { The surface contamination average value for } A D 1 \\
\text { for December is out of } 1 \text { ine with those for } \\
\text { November and January. }\end{array}$ & $\begin{array}{l}\text { The value in question is correct. } \\
\text { It is consistent with December } \\
\text { values for adjacent locations. }\end{array}$ \\
\hline 32. & $\mathrm{DP}$ & & $\begin{array}{l}\text { Converting surface contamination values to index } \\
\text { numbers more readily shows the relationship } \\
\text { between contamination in different geographic } \\
\text { areas. }\end{array}$ & $\begin{array}{l}\text { The requested transformation is } \\
\text { outside the scope of the report. } \\
\text { See response to comment } 30 \text {. }\end{array}$ \\
\hline 33. & $\begin{array}{l}\text { John E. } \\
\text { Ti11 }\end{array}$ & & $\begin{array}{l}\text { I do not expect that the doses will change signi- } \\
\text { ficantly, but this is something we will have to } \\
\text { repeat after we get the correct met data for } \\
1944-47 \text {. }\end{array}$ & $\begin{array}{l}\text { Cursory examination of the results } \\
\text { of model calculations made with } \\
\text { the 19B3-1987 and } 1944-1947 \text { mete- } \\
\text { orological data sets doesn't indi- } \\
\text { cate that use of the } 1944-1947 \\
\text { meteorological data in the Phase I } \\
\text { study would have changed the Phase } \\
\text { I conclusions. }\end{array}$ \\
\hline
\end{tabular}

Document Title Atmospheric Transport Modeling and Input Data for Phase I of the HEDR Project 


\section{SUMMARY OF TSP COMMENTS AND BATTELLE RESPONSES}

Document Number PNL-7199. HEDR

Document Title Atmospheric Transport Model ing and Input Data for Phase I of the HEDR Project

\begin{tabular}{|c|c|c|c|c|}
\hline $\begin{array}{l}\text { Comment } \\
\text { Number }\end{array}$ & Commenter & $\begin{array}{c}\text { Page, } \\
\text { Paragraph }\end{array}$ & Comment Summary & Resolution \\
\hline 34. & $\mathrm{JET}$ & & $\begin{array}{l}\text { Add equations and figures to take the reader } \\
\text { through the input variables, the normalization } \\
\text { process, and how the results will be used. }\end{array}$ & $\begin{array}{l}\text { The requested information is out- } \\
\text { side the scope of this report. } \\
\text { However, much of it was included } \\
\text { in the MESOILT2 code documentation } \\
\text { (PNL-7340 HEDR). }\end{array}$ \\
\hline 35. & $\mathrm{JET}$ & Pages $v, 1$ & $\begin{array}{l}\text { Don't rule out the possibility that we will use } \\
\text { this model. }\end{array}$ & $\begin{array}{l}\text { MESOILT2 provides the general } \\
\text { framework for the final model. } \\
\text { However, many of the details of } \\
\text { the model will change to enhance } \\
\text { the technical credibility of the } \\
\text { model and to permit estimates of } \\
\text { uncertainty to be included in } \\
\text { model calculations. }\end{array}$ \\
\hline 36. & $\mathrm{JET}$ & & $\begin{array}{l}\text { Although the puff model is used here, it is the } \\
\text { summation of puffs over time that counts. This } \\
\text { point should be stressed. What we are most } \\
\text { interested in is the time-integrated concentra- } \\
\text { tion in air or on land surface. }\end{array}$ & $\begin{array}{l}\text { The comment is correct. In addi- } \\
\text { tion, the literature indicates } \\
\text { that uncertainty in model results } \\
\text { decreases as the integration time } \\
\text { increases. }\end{array}$ \\
\hline 37. & JET & $\begin{array}{l}\text { Page } 4 \\
\text { para } 4\end{array}$ & $\begin{array}{l}\text { The rationale for the domain being shifted to the } \\
\text { east is not clear, especially since we wanted to } \\
\text { specifically address the } 10 \text {-county area. }\end{array}$ & $\begin{array}{l}\text { The offset was based on consider- } \\
\text { ation of prevailing wind patterns } \\
\text { and topography. The discussion of } \\
\text { the domain has been expanded to } \\
\text { include the rationale. }\end{array}$ \\
\hline 38. & , JFT & $\begin{array}{l}\text { Page } 6 \\
\text { para } 5\end{array}$ & $\begin{array}{l}\text { Will we have the } 1944-47 \text { data in time to include } \\
\text { an analysis illustrating the potential effect on } \\
\text { the results of Phase I from their insertion? If } \\
\text { at all possible, we need to do this. }\end{array}$ & See response to comment 17 \\
\hline
\end{tabular}


SUMMARY OF TSP COMMENTS AND BATTELLE RESPONSES

Document Number PNL-7199 HEDR

Oocument Title Atmospheric Transport Modeling and Input Data for Phase I of the HEDR Project

\begin{tabular}{|c|c|c|c|c|}
\hline $\begin{array}{l}\text { Comment } \\
\text { Number }\end{array}$ & Commenter & $\begin{array}{c}\text { Page, } \\
\text { Paragraph }\end{array}$ & Comment Summary & Resolution \\
\hline 39. & JET & $\begin{array}{l}\text { Page } 9 \\
\text { Table } 1\end{array}$ & $\begin{array}{l}\text { Make it clear in the title that these results are } \\
\text { for } 1983-87 \text { met data. }\end{array}$ & Revised title. \\
\hline 40. & $\mathrm{JET}$ & $\begin{array}{l}\text { Page } 8 \text {, } \\
\text { line } 2 \\
\text { (and } \\
\text { elsewhere) }\end{array}$ & $\begin{array}{l}\text { Use }{ }^{131} \text { I format. Further, I know gasses is a } \\
\text { correct plural spelling, but I think this is the } \\
\text { first time I have seen it used. I would be } \\
\text { interested in other opinions, but I prefer gases. }\end{array}$ & $\begin{array}{l}\text { We have chosen to spell out the } \\
\text { names of radionuclides throughout } \\
\text { text for clarity. "Gasses" has } \\
\text { been replaced by "gases." }\end{array}$ \\
\hline 41. & JET & $\begin{array}{l}\text { Page } 10, \\
\text { para } 1\end{array}$ & $\begin{array}{l}\text { Add references for the stack flow rate and offgas } \\
\text { temperature. Also, was there a variation in } \\
\text { offgas temperature based on time of year or } \\
\text { throughput? Show references. }\end{array}$ & Text revised. \\
\hline 42. & JET & Page 11 & $\begin{array}{l}\text { Normalization explanation is not very clear. Why } \\
\text { is } 1000 \mathrm{C} \text { i used, then normalization to } 1 \mathrm{Ci} \text { ? } \\
\text { A] so, a figure (block diagram) explaining what } \\
\text { you are doing would be helpful. }\end{array}$ & $\begin{array}{l}\text { Text revised to include an } \\
\text { explanation. }\end{array}$ \\
\hline 43. & $\mathrm{JET}$ & $\begin{array}{l}\text { Page 22, } \\
\text { Table } 3\end{array}$ & $\begin{array}{l}\text { Why report these data in logarithms? It is } \\
\text { difficult to interpret. Unless there is a good } \\
\text { reason, standard values (using E format) should } \\
\text { be listed. }\end{array}$ & $\begin{array}{l}\text { The data are reported in loga- } \\
\text { rithms because they were trans- } \\
\text { ferred to the dose assessment task } \\
\text { in that form. The tables are } \\
\text { listings of computer files with } \\
\text { appropriate headings added. The } \\
\text { discussion on pages } 20-21 \text { was } \\
\text { expanded. }\end{array}$ \\
\hline 44. & JET & & $\begin{array}{l}\text { Plot average } X / Q \text { values on the county map and } \\
\text { convert these to isopleths for each month or for } \\
\text { quarters. }\end{array}$ & $\begin{array}{l}\text { The requested figures are outside } \\
\text { the scope of this report. See } \\
\text { response to comment } 30 \text {. }\end{array}$ \\
\hline
\end{tabular}


SUMMARY OF TSP COMMENTS AND BATTELLE RESPONSES

Document Number PNL-7199 HEDR

\begin{tabular}{|c|c|c|c|c|}
\hline $\begin{array}{l}\text { Comment } \\
\text { Number }\end{array}$ & Commenter & $\begin{array}{l}\text { Page, } \\
\text { Paragraph }\end{array}$ & Comment Summary & Resolution \\
\hline 45. & $\begin{array}{l}\text { Bernard } \\
\text { Shleien }\end{array}$ & & $\begin{array}{l}\text { The report provides no information of substance } \\
\text { as to preliminary results. Should give actual } \\
\text { data on parameters specific to Phase I, and } \\
\text { uncertainties associated with the models used. }\end{array}$ & $\begin{array}{l}\text { The report describes the atmos- } \\
\text { pheric model input and output for } \\
\text { the Phase I calculations. } \\
\text { Uncertainties in the mode } 1 \text { results } \\
\text { are included in the output based } \\
\text { on evaluation of performance of } \\
\text { the predecessor model in estimat- } \\
\text { ing monthly-average krypton-85 } \\
\text { concentrations at Hanford, the } \\
\text { interannual variability of model } \\
\text { estimates of monthly-average air } \\
\text { concentrations, and the spatial } \\
\text { variability of concentration esti- } \\
\text { mates within census districts. } \\
\text { MESoILT2 does not have mechanisms } \\
\text { to incorporate estimates of model } \\
\text { parameter uncertainty in its cal- } \\
\text { culations or to generate estimates } \\
\text { of model uncertainty. }\end{array}$ \\
\hline 46. & BS & & $\begin{array}{l}\text { Without some information on the uncertainties, or } \\
\text { the variance that could be expected due to } \\
\text { specific decision points, one cannot judge the } \\
\text { utility of the results for Phase I. }\end{array}$ & $\begin{array}{l}\text { The purpose of the Phase I cal- } \\
\text { culations was to demonstrate that } \\
\text { data and models are available to } \\
\text { make the calculations needed for } \\
\text { dose reconstruction. }\end{array}$ \\
\hline 47. & $\begin{array}{l}\text { Vietchau } \\
\text { Nauyen }\end{array}$ & & $\begin{array}{l}\text { Given the underlying assumptions of the } \\
\text { Lagrangian formulation (which led to the } \\
\text { fundamental MESOI Gaussian solution), } \\
\text { applicability of the MESOI formulation to the } \\
\text { HEDR Project domain must be justified. }\end{array}$ & $\begin{array}{l}\text { The selection of the atmospheric } \\
\text { modeling approach is discussed in } \\
\text { PNL-7998 and was discussed again } \\
\text { at a TSP workshop in June } 1990 \text {. } \\
\text { The workshop participates (includ- } \\
\text { ing the outside experts). agreed }\end{array}$ \\
\hline
\end{tabular}




\begin{tabular}{|c|c|c|c|c|}
\hline $\begin{array}{l}\text { Comment } \\
\text { Number }\end{array}$ & Commenter & $\begin{array}{c}\text { Page, } \\
\text { Paragraph }\end{array}$ & Comment Summary & Resolution \\
\hline & & & & $\begin{array}{l}\text { that a puff model provides an } \\
\text { appropriate framework of transport } \\
\text { and diffusion calculations for the } \\
\text { Hanford Environmental Dose Recon- } \\
\text { struction Project. }\end{array}$ \\
\hline 48. & VN & & $\begin{array}{l}\text { The validity of the assumptions of the slender } \\
\text { plume approximation needs to be verified for the } \\
\text { HEDR project. }\end{array}$ & $\begin{array}{l}\text { The puff model does not rely on } \\
\text { the slender plume approximation. } \\
\text { However, all modeling assumptions } \\
\text { are being re-evaluated as a part } \\
\text { of the model revision process. }\end{array}$ \\
\hline 49. & VN & & $\begin{array}{l}\text { Further explanation/justification is required for } \\
\text { 1) the weighting process used in the calculation } \\
\text { of sector-averaged exposure and 2) the assumption } \\
\text { of joint probability of wind direction transport } \\
\text { into a sector, wind speed and stability class. }\end{array}$ & $\begin{array}{l}\text { Sector-average exposures are not } \\
\text { calculated anywhere in MESOILT2, } \\
\text { and the model does not make use of } \\
\text { joint probability distributions. } \\
\text { The model uses actual observed } \\
\text { meteorological data in its } \\
\text { calculations. }\end{array}$ \\
\hline 50. & VN & & $\begin{array}{l}\text { The proposed atmospheric modeling approach } \\
\text { requires many computational assumptions, which } \\
\text { must be shown to be consistent with the basic } \\
\text { physics principles and site conditions. }\end{array}$ & $\begin{array}{l}\text { The approach is consistent with } \\
\text { basic physical principles and the } \\
\text { available data. }\end{array}$ \\
\hline 51. & VN & & $\begin{array}{l}\text { The slender plume approximation will undoubtedly } \\
\text { underestimate the concentration distant from the } \\
\text { area covered by the progression of the plume. } \\
\text { This underestimation is inherent in the basic } \\
\text { formulation of the MESOI code. }\end{array}$ & $\begin{array}{l}\text { The assertion is not correct. } \\
\text { Both overestimates and under- } \\
\text { estimates of time-integrated air } \\
\text { concentrations and deposition are } \\
\text { possible at all distances. }\end{array}$ \\
\hline
\end{tabular}


SUMMARY OF TSP COMMENTS AND BATTELLE RESPONSES

Document Number PNL-7199 HEDR
Document Title Atmospheric Transport Modeling and Input Data for Phase I of the HEDR Project

\begin{tabular}{|c|c|c|c|c|}
\hline $\begin{array}{l}\text { Comment } \\
\text { Number }\end{array}$ & Commenter & $\begin{array}{l}\text { Page, } \\
\text { Paragraph } \\
\end{array}$ & Comment Summary & Resolution \\
\hline 52. & VN & & $\begin{array}{l}\text { Using the NRC approximation would result in } \\
\text { overestimation of concentrations in the near- } \\
\text { field and near the puff trajectory, and under- } \\
\text { estimation of values far-field and/or away from } \\
\text { the puff trajectory. }\end{array}$ & $\begin{array}{l}\text { The assertion is not correct. } \\
\text { However, methods used to estimate } \\
\text { diffusion will be re-evaluated as } \\
\text { part of the model revision } \\
\text { process. }\end{array}$ \\
\hline 53. & VN & & $\begin{array}{l}\text { The concept of sector averaging, as presented in } \\
\text { this report, seems to be arbitrary and has no } \\
\text { physical foundation. }\end{array}$ & $\begin{array}{l}\text { MESOILT2 does not incorporate any } \\
\text { sector averaging. }\end{array}$ \\
\hline 54. & VN & & $\begin{array}{l}\text { Due to the rapid chemical reactions of some } \\
\text { released radionuclides in the atmosphere, the } \\
\text { fundamental principles used by MESOI and the } \\
\text { underlying assumptions concerning the advection } \\
\text { time scale and the chemical reaction time scale } \\
\text { must be reexamined. }\end{array}$ & $\begin{array}{l}\text { All modeling assumptions will be } \\
\text { reexamined as part of the model } \\
\text { revision process. }\end{array}$ \\
\hline 55. & VN & & $\begin{array}{l}\text { A computational mechanism must be provided to } \\
\text { reconcile all possible errors resulting from the } \\
\text { assumptions and approximations made in the } \\
\text { atmospheric transport modeling approach using } \\
\text { MESOI. }\end{array}$ & $\begin{array}{l}\text { The revised model will include } \\
\text { mechanisms to account for uncer- } \\
\text { tainty in the model input. }\end{array}$ \\
\hline 56. & VN & & $\begin{array}{l}\text { Sensitivity analyses should be performed to } \\
\text { establish the variations which are inherently } \\
\text { allowed by the intrinsic formulation of the code. } \\
\text { Sensitivity studies for each sigma-2 } \\
\text { parameterization is also recommended. }\end{array}$ & $\begin{array}{l}\text { The sensitivity of the revised } \\
\text { model to the changes in model } \\
\text { components will be evaluated. }\end{array}$ \\
\hline 57. & VN & & $\begin{array}{l}\text { All sigma-z parameterizations should be tested } \\
\text { and compared against data and against each other. }\end{array}$ & $\begin{array}{l}\text { Alternative methods for estimating } \\
\text { diffusion are being evaluated. }\end{array}$ \\
\hline
\end{tabular}




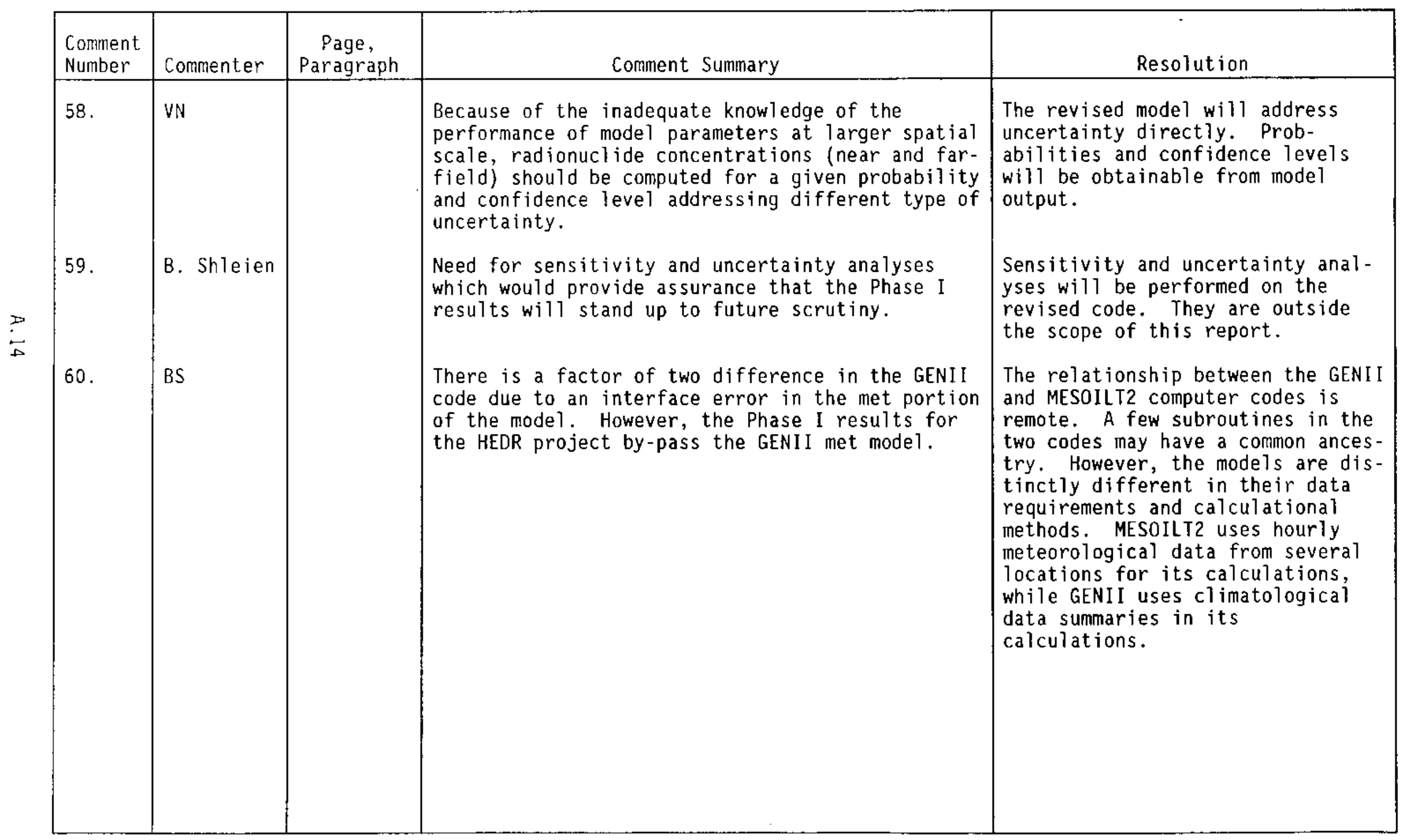




\section{DISTRIBUTION}

No. of

Copjes

\section{QFFSITE}

18 Technical Steering Panel

D.S. Barth

University of Nevada

4505 Maryland Parkway

Las Vegas, NV 89154

W.A. Bishop

2503 Wedgewood Court SE

Olympia, WA 98501

M.L. Blazek

Oregon Department of Energy

625 Marion Street N.E.

Salem, OR 97303

G. G. Caldwell

Director

Tulsa City-County Health Dept.

4616 East 15th Street

Tulsa, OK 74112

S.N. Davis

Dept. of Hydrology \& Water Resources

81dg. 11

University of Arizona

Tucson, AZ 85721

N.J. Germond

224 Iron Mountain B7vd.

Lake Ozwego, OR 97034

P.C. Klingeman

Civil Engineering Dept.

Oregon State University

Corvallis, OR 97331-2302
No. of

Copjes
K.J. Kopecky

Fred Hutchinson Cancer Research Center

1124 Columbia Street

Seattle, WA 98104

R.L. Morrill

Dept. of Geography

DP. 10

University of Washington

Seattle, WA 98195

A.H. Murphy

c/o Climate Analyses Center

National Weather Service

W/NMC51, WWB, Room 604

Washington, DC 20233

V. Nguyen

EWA, Inc.

I33 lst Ave. N.

Minneapolis, MN 55401

D.W. Price

Agricultural Economics

Hulbert Hall Room 211

Washington State University

Pullman, WA 99164-6210

M.A. Robkin

Radiological Sciences SB-75

University of Washington

Seattle, WA 98195

G.S. Roessler

Rt. 1, Box 139H

Elysian, MN 56028 
No. of

Copies

B. Shleien

2421 Homestead Drive

Silver Springs, MD 20902

A.P. Slickpoo, Sr.

P.0. Box 331

809 Nez Perce Lane

Kamiah, ID 83536

J.E. Ti11

Rt. 2 Box 122

Neeses, SC 29107

D.E. Walker, Jr.

P.0. $80 \times 4147$

Boulder, C0 80306

2 DOE Office of Scientific and Technical Information

Technical Information Center P.0. Box 62

Oak Ridge, TN 37830

18 K. Charlee

Office of Nuclear Waste Mgmt.

Department of Ecology

99 South Sound Center

Mail Stop PV-11

01 ympia, WA 98504

3 M. Sage

Centers for Disease Control 1600 Clifton Road

Atlanta, GA 30333

J. Thomas

HEAL

1720 N. Ash

Spokane, WA 99205
No. of

Copies

\section{ONSITE}

1 Hanford Environmental Health Foundation

S.E. Dietert H1-03

2 Westinghouse Hanford Company

C.D. Carpenter H4-15

D.E. Wood H4-5l

8 DOE Richland 0perations

T. A. Bauman, COM

A7 -75

R. F. Brich, SED

A5 -55

R. A. Holten, SED

A5 -55

J. R. Hunter, OPD

A6- 53

J. J. Keating, AMS

A5 -54

R. M. Rosselii, AMA

A7 -70

R. R. Tibbatts, FRD

A7 -89

M. W. Tiernan, SED

A5 -55

37 Pacific Northwest Laboratory

K. W. Burk

J. M. Daer (10)

K6-03

M. D. Freshley

$K 6-89$

R. 0 . Gilbert

$\mathrm{K} 6-77$

W. A. Glass (6)

$\mathrm{K} 7-34$

S. P. Gydesen

K4 -13

A. H. McMakin

P8-55

B. A. Napier

K6-86

J. V. Ransde 11 (4)

K3 -54

W. L. Templeton

K6-03

S. B. Yabusaki

$\mathrm{K} 1-30$

Publishing Coordination

Records Center

Al -65

Technical Librar

(2)
$\mathrm{KI}-11$

$\mathrm{K} 3-70$

P8-55 